\title{
Plasma-assisted cleaning of \\ extreme UV optics
}

Alexandr Dolgov

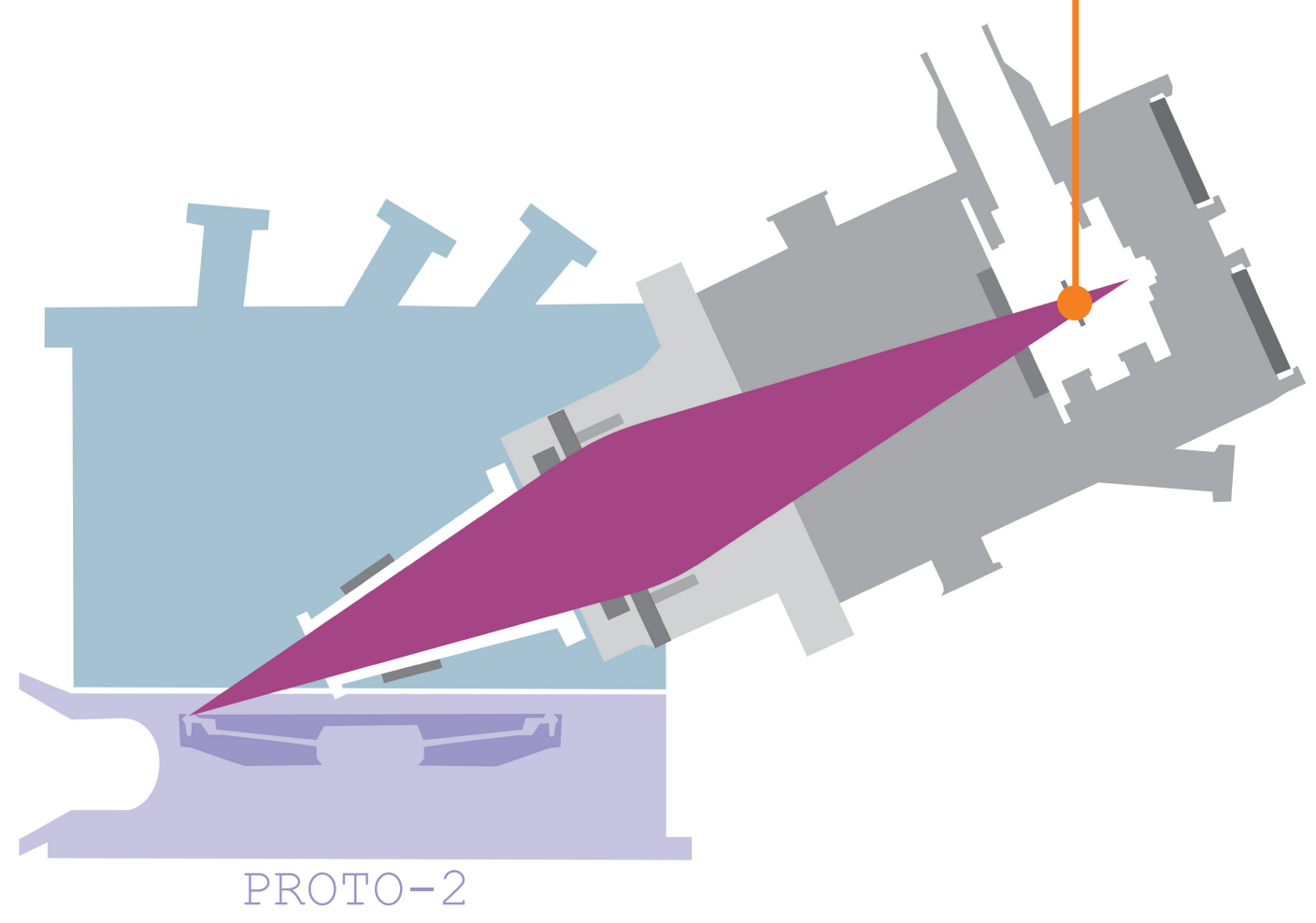




\section{Plasma-assisted cleaning \\ of}

\section{extreme UV optics}

Alexandr A. Dolgov 
Graduation committee:

Chairman:

Prof. dr. ir. J. W. M. Hilgenkamp University of Twente, TNW

Secretary:

Prof. dr. ir. J. W. M. Hilgenkamp University of Twente, TNW

Supervisor:

Prof. dr. F. Bijkerk

University of Twente, TNW

Co-supervisor:

Dr. C. J. Lee

Fontys Eindhoven

Members:

Prof. dr. ir. H. J. W. Zandvliet

University of Twente, TNW

Prof. dr. P. W. H. Pinkse

University of Twente, TNW

Prof. dr. ir. V. Y. Banine

Technische Universiteit

Eindhoven

Dr. D. V. Lopaev

Moscow State University

\section{PLASMA-ASSISTED CLEANING OF EXTREME UV OPTICS}

Alexandr A. Dolgov

PhD thesis, University of Twente, Enschede, The Netherlands

With references -With summary in English.

ISBN: 978-90-365-4506-8

DOI: $10.3990 / 1.9789036545068$

Published by the Industrial Focus Group XUV Optics, University of Twente, Enschede, The Netherlands

Printed by Ipskamp Printing, Enschede, The Netherlands

(C) Alexandr A. Dolgov(2018)

All rights reserved. 
OF

\title{
EXTREME UV OPTICS
}

\author{
DISSERTATION \\ to obtain \\ the degree of doctor at the University of Twente, \\ on the authority of the rector magnificus \\ prof. dr. T. T. M. Palstra \\ on account of the decision of the graduation committee, \\ to be publicly defended \\ on Thursday the $1^{\text {st }}$ of March 2018 at 14:45
}

by

\section{Alexandr Alexeevich Dolgov}

\author{
born on $26^{\text {th }}$ of July 1985 \\ in Kaunas, Lithuania
}


This dissertation has been approved by the supervisor:

Prof. dr. F. Bijkerk

and the co-supervisor:

Dr. C. J. Lee

ISBN: 978-90-365-4506-8

(c) Alexandr Alexeevich Dolgov (2018) 


\section{This thesis based on following publications:}

Chapter 2. - A Dolgov, O Yakushev, A Abrikosov, E Snegirev, VM Krivtsun, CJ Lee, F Bijkerk Extreme ultraviolet (EUV)source and ultra-high vacuum chamber for studying EUV-induced processes. Plasma sources science and technology 24 (3), 035003 (2015)

Chapter 3. - A Abrikosov, V Reshetnyak, D Astakhov, A Dolgov, O Yakushev, D Lopaev, and V Krivtsun Numerical simulations based on probe measurements in EUV-induced hydrogen plasma, Plasma Sources Science and Technology, Volume 26, Number 4 (2017)

Chapter 4. - A Dolgov, D Lopaev, T Rachimova, A Kovalev, A Vasil'eva, C J Lee, V M Krivtsun, $\mathrm{O}$ Yakushev and F Bijkerk Comparison of $\mathrm{H}_{2}$ and $\mathrm{He}$ carbon cleaning mechanisms in extreme ultraviolet induced and surface wave discharge plasmas. J. Phys. D: Appl. Phys. 47 (2014)

Chapter 5. - A Dolgov, D Lopaev, CJ Lee, E Zoethout, V Medvedev, O Yakushev, F Bijkerk Characterization of carbon contamination under ion and hot atom bombardment in a tin-plasma extreme ultraviolet light source, Applied Surface Science 353, 708-713 (2015)

Chapter 6.- A Dolgov, A Abrikosov, C J Lee, M van Kampen, V M Krivtsun, O Yakushev, D Lopaev and F Bijkerk Plasma-assisted oxide removal from ruthenium coated EUV optics. Journal of Applied Physics submitted (2017)

This work is part of the research programme 'Controlling photon and plasma induced processes at EUV optical surfaces (CP3E)' of the 'Stichting voor Fundamenteel Onderzoek der Materie (FOM)', which is part of and financially supported by the 'Nederlandse Organisatie voor Wetenschappelijk Onderzoek (NWO)'. The CP3E programme is also co-financed by Carl Zeiss SMT and ASML, and carried out in the Industrial Focus Group XUV Optics, which belongs to the MESA+ Institute for Nanotechnology at the University of Twente. The infrastructure for this programme is also financially supported by the Province of Overijssel. 


\section{Contents}

1.Introduction 8

1.1Cleaning methods for multilayer optics $\quad 12$

1.2 Anticipated method of cleaning of optics $\quad 12$

1.3 EUV radiation driven cleaning $\quad 15$

References

2. Extreme ultraviolet source and ultra high vacuum chamber for studying EUV-induced processes

Abstract

Introduction

2.1 Setup

2.1.1 UHV chamber $\quad 26$

2.2 Study of the EUV-induced plasma 29

2.2.2 Probe measurement scheme 29

2.3 Experimental results $\quad 32$

2.4 Probe measurements $\quad 34$

2.5 Conclusion $\quad 36$

References

38

3. Numerical simulations based on probe measurements

in EUV-induced hydrogen plasma

Abstract.

Introduction 39

3.1 Experimental setup 43

3.2 Results and discussion $\quad 45$

3.2.1 Photocurrent 46

3.2.2 Probe measurements $\quad 48$

3.3 Numerical modeling $\quad 49$

3.3.1 Validating the model input 49

3.3.2 EUV-induced plasma formation under different conditions 51

3.4 Conclusion $\quad 56$

References 58

4. Comparison of $\mathrm{H}_{2}$ and $\mathrm{He}$ carbon cleaning mechanisms

in extreme ultraviolet induced and surface wave discharge plasmas

Abstract. 
Introduction

4.1Samples and diagnostics of amorphous carbon 62

4.2 EUV-induced plasma 63

4.3 Low-pressure SWD discharge plasma 68

4.4 Discussion $\quad 73$

4.5 Conclusion $\quad 83$

References $\quad 85$

5.Characterization of carbon contamination under ion and hot atom bombardment in a tin-plasma extreme ultraviolet light source

Abstract

Introduction

5.1. Experiment

5.1.1 Film preparation

91

5.1.2 Analysis

5.2 Conclusion

6. Plasma-assisted oxide removal from ruthenium coated EUV optics

Abstract

Introduction

6.1 Oxidized ruthenium samples

6.2 Evolution of oxidation and reduction of ruthenium in water/hydrogen medium

6.3 Plasma-assisted ruthenium reduction

6.4 EUV-induced plasma experiments

6.5 Discussion

6.6 Conclusions

134

References

7. Summary:

8. Valorisation

9. Appendix1

References

Acknowledgements 


\section{Introduction}

The next generation of photolithography, extreme ultraviolet (EUV) lithography, makes use of $13.5 \mathrm{~nm}$ radiation, emitted by a pulsed hot high-density tin plasma [1]. The ionizing photon flux, and vacuum requirements create a challenging operating environment. One of the challenges is to maintain the reflectivity, figure, and positioning of the optics. Because of high EUV absorption, the optical elements must be reflective rather than transmissive and must use multilayer mirrors (MLM) of the highest quality.

MLMs consist of approximately $50 \mathrm{Mo} / \mathrm{Si}$ bi-layers, stacked to form an artificial 1D Bragg crystal. The choice of the mirror material has been determined by meeting the simultaneous requirements for maximum possible optical contrast between the layers (i.e., the maximum possible difference between the real parts of the refractive indices of the two materials), and the minimum possible absorption (i.e., the minimum possible imaginary part of the refractive index). In addition to these requirements, the two materials should form smooth, sharp, and long lasting interfaces. Mo and $\mathrm{Si}$ form a pair of materials ensuring the maximum practical reflectivity for a mirror designed to operate at $13.5 \mathrm{~nm}$ wavelength (92 eV photon energy). The maximum theoretical reflectivity of a $\mathrm{Mo} / \mathrm{Si} \mathrm{MLM}$ is $74 \%$, while the experimentally achieved value is $\sim 70 \%$ [2-6]. A thorough review of these mirrors is given in [7]. Multilayer mirrors in EUVL are expensive high-technology items. Since it is expensive to manufacture such optics, the lifetime of $\mathrm{Mo} / \mathrm{Si}$ multilayers is one of the critical parameters for evaluating their performance. 
Previous research has demonstrated that MLMs may lose their reflectivity due to their surfaces becoming contaminated. [8,9]. For a single optical element, a minor loss in reflectivity may not be significant. However, for imaging systems that consist of multiple elements, it is critical that peak and uniform reflectivity is maintained. For instance, an EUVL optical system may consist of $\sim 10$ multilayer mirrors (figure 1), if the reflectivity of each mirror drops by $1 \%$, then the total throughput drops by $15 \%$. Furthermore, if the reflectivity loss is unevenly distributed over the surface of the mirrors, then the resolution of the image may be adversely effected.

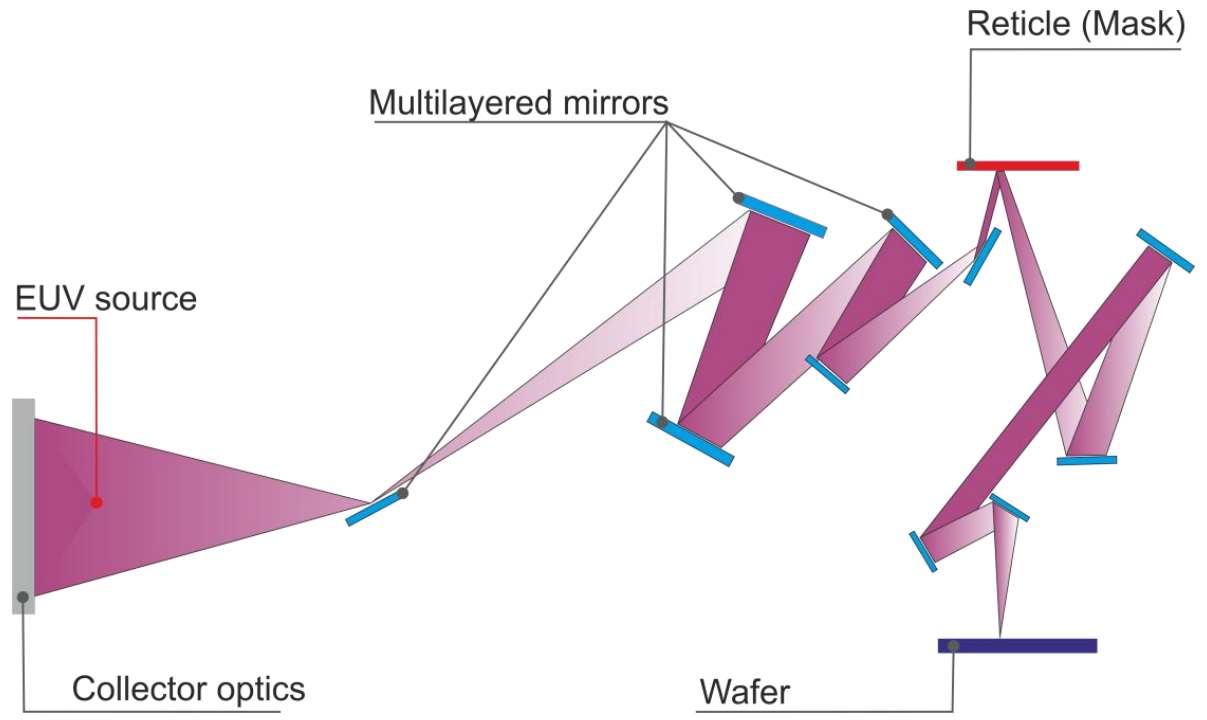

Figure 1. Example of an EUV lithography imaging scheme. The apparatus is comprised of a $13.5 \mathrm{~nm}$ light source; collector optics for the collection and focusing of source radiation at the intermediary focus; illumination optics to obtain appropriate illumination for the mask, and projection optics to accurately project the mask pattern onto the photoresist-coated silicon wafer.

Reflectivity loss due to contamination should be viewed from the perspective of the expected lifetime of the optical element. EUVL 
requires a lifetime of $3 \cdot 10^{4}$ hours for projection optics MLMs [10,11], while, on the other hand, collector optics are swappable and only expected to last $\sim 3000$ hours. Likewise, the mask, which also has a MLM substrate, may only be used for a few hundred hours. Contamination is generally caused by hydrocarbons and water molecules that decompose under the action of EUV radiation, causing an amorphous carbon film or oxide layer to form on the mirror surface.

The formation of such films on the MLM surface is the main cause of lifetime reduction for EUV optics. For example [12, 8], a carbon film just a few nm thick would already cause significant loss of MLM reflectivity. In vacuum conditions, the partial pressure of hydrocarbons defines the rate of amorphous carbon growth. The growth rate of the carbon layer can be quite high, ranging from 0.001 $\mathrm{nm} / \mathrm{hr}$ to $0.01 \mathrm{~nm} / \mathrm{hr}$, depending on the precise EUV illumination and partial pressures $[13,14]$. Over the range of these growth rates, it is clear that only the mask may not be effected by carbon growth.

To maximise the lifetime of MLMs, oxidation of the top protective coating [14] should also be controlled. Although oxidation rates have not been as thoroughly investigated as carbon growth rates, a rough estimate has been made. For instance, at $2 \cdot 10^{-6}$ Torr $\mathrm{H}_{2} \mathrm{O}$ pressure the oxidation rate is estimated to range from $0.016 \mathrm{~nm} / \mathrm{h}$ up to 0.11 $\mathrm{nm} / \mathrm{h}$ and depends on the EUV radiation pulse width, dose and intensity. Under intense EUV radiation, partially or fully dissociated water forms reactive species ( $\mathrm{OH}$ groups, $\mathrm{O}$ atoms, $\mathrm{H}$ atoms). Reactive species allow surface oxidation to proceed either directly, or via subsequent reactions during exposure.

Reflectivity loss of a multilayer mirror caused by oxidation of the protective layer has been measured in ref [15]. For these 
experiments, a short exposure (4-5 hours) at a high water partial pressure $\left(5 \cdot 10^{-6}\right.$ Torr) showed a reflectivity drop of $2 \%$. Although the water vapor pressure in an actual lithography apparatus would, doubtlessly, be less than the test pressure, the oxidation rate should also decrease by a factor of $10^{4}$ to meet lifetime requirements, which seems unlikely. Thus, any in situ mirror cleaning should also be able to remove or reduce surface oxides.

Oxidation of ruthenium surfaces is often described by a model, which was presented in detail in [16]. The mathematical model of the oxidation describes the main steps: adsorption of water molecules, dissociation, surface diffusion etc., and presents the estimated dependence of the thickness of oxide layer on radiation dose. Calculations show that oxidation predominantly occurs in the presence of high intensity radiation for the case relevant for a lithography machine. The model suggests that surface oxidation saturates after the formation of one monolayer of atomic oxygen. According to the model, high intensity EUV produces atomic oxygen in excess of one monolayer, but the excess dissociatively desorbs from the surface, rather than contributing to the formation of subsurface oxide.

Furthermore, the balance between oxidation and reduction under EUV radiation, and, consequently, in the presence of ions and radical species has not been investigated.

Besides the above-mentioned types of contamination, there are other types of contamination, such as tin (Sn). Sn is used as a source of EUV radiation, and may contaminate collector mirrors. These three types of contamination are discussed in more detail in this thesis. 


\subsection{Cleaning methods for multilayer optics.}

As can be seen by comparing the growth rates of contaminants with the expected lifetime of the various optics, contamination must be actively controlled. To control contamination of optical surfaces, a technology that efficiently and precisely cleans MLM surfaces is required. In a technically perfect situation, such a cleaning procedure should be realized without any interruptions to machine operation, i.e. in situ and in line.

Atomic hydrogen is currently used to remove different types of contaminants, because the majority of basic hydrogen compounds are volatile. However, atomic hydrogen is not a very effective in all circumstances: for example, the removal of surface oxides. In addition, the efficiency of atomic hydrogen cleaning of amorphous carbon-the most common contaminant-is extremely low [17], particularly in comparison with the surface recombination probabilities [18].

New cleaning methods, suitable for the conditions found in EUV lithography, must be developed.

\subsection{Anticipated Method of Cleaning of Optics}

Prior to looking closely at alternative cleaning methods, the requirements for mirror cleaning should be reviewed. The requirements essentially emerge from the conditions discussed above.

- A cleaning method must work in-situ, i.e. cleaning without interrupting wafer printing. 
- Cleaning must be performed at the speed of about the growth rate $(0.01-0.1 \mathrm{~nm} / \mathrm{h})$ or faster.

- Cleaning should be highly selective. Cleaning selectivity is given by the ratio of carbon atoms removed to cap-layer (e.g., ruthenium) atoms removed. Provided that the required mirror lifetime is $3 \cdot 10^{4}$ hours, the Ru layer thickness is $2 \mathrm{~nm}$, and the required cleaning rate is at least $0.1 \mathrm{~nm} / \mathrm{h}$, the required cleaning selectivity $\xi_{R u}^{C}$ is as follows:

$$
\xi_{R u}^{C}=\frac{R_{C}}{R_{R u}}>10^{3} \div 10^{4}
$$

Where $R_{C}$ - carbon removal rate, $R_{R u}$ - Ru removal rate. This estimation of the selectivity for carbon with reference to a Ru top layer is based on the assumption that the protective capping layer should last the lifetime of the mirror. Atomic hydrogen, or hot-wire cleaning, is currently considered as one of the most feasible methods applied $[9,19,20,21]$. The method essentially involves flowing hydrogen over a tungsten wire heated to $\sim 2000^{\circ} \mathrm{C}$. When subjected to such a temperature, hydrogen molecules largely dissociate into atoms. The flow of fast-moving atoms hits the surface of the mirror and may react with carbon to form volatile species that leave the surface of the mirror. Despite the obvious benefits of this method in terms of removal of contamination, the method has certain drawbacks. First, probability of removal of one carbon atom per atom is very low $\left(\sim 10^{-6}\right)$, meaning the efficiency is low for carbon, and is even lower for other forms of contamination, such as oxide formation [22]. Second, the mirror may be contaminated with $\mathrm{W}$ atoms that boil off from the surface of the heated wire. As a result, atomic hydrogen cleaning barely satisfies the above criteria, and other solutions to this issue must be considered. 
An alternative to using hydrogen radicals is the use of plasmaassisted cleaning. At the moment, RF plasma is typically used for this. The effectiveness of such cleaning for carbon removal is well described in [17] for a hydrogen plasma, generated in a $40 \mathrm{MHz}$ capacitively coupled plasma (CCP) discharge. In this case the energy of ions incident onto the sample surface does not exceed $20 \mathrm{eV}$ (i.e. plasma potential). According to plasma probe measurements, maximum plasma density corresponded to ne $10^{10} \mathrm{~cm}^{-3}$ and ion flux varied in the range of $1 \cdot 10^{14}$ to $5 \cdot 10^{14} \mathrm{ion} / \mathrm{cm}^{2} \cdot$ s.

From the data measured in the discharge afterglow, a removal probability up to $10^{-5} \mathrm{C}$ atoms per incident $\mathrm{H}$ atom was estimated. However, the $\mathrm{C}$ atom removal probability per incident hydrogen ion was found to be at least 0.1 . This experiment promises high cleaning efficiency while maintaining the selectivity of atomic hydrogen.

The relatively high ion energy of traditional (inductive or capacitive coupling) plasma do not have the selectivity required to meet MLM lifetime requirements. In practice this means that the ion energy must be kept below $20 \mathrm{eV}$. In the case of ICP or CCP discharge, selectivity is difficult to achieve because of the rather high plasma potential. Furthermore, the placement of electrodes for plasma ignition is impractical in many cases.

The alternative considered in this thesis is to use the plasma generated by ionization due to EUV radiation to etch contaminants from the surface. However, as discussed below, EUV-induced plasmas operate in an entirely different energy and density regime, so it is not clear that such a plasma will efficiently remove contaminants from optical surfaces. The focus of this thesis was to characterize the EUV plasma, and determine under what conditions it can be used to etch contaminants. And, finally to understand the 
etching process in detail. To achieve this, experiments in conditions similar to those found in EUVL, with various environment gases and pressures, must be carried out.

\subsection{EUV radiation driven cleaning.}

EUV radiation is radiation with photon energies from about 30 to 250 $\mathrm{eV}$ [1]. Due to such high energies, photons ionize a fraction of the background gas. Besides gas photoionization, EUV photons can generate energetic photoelectrons from any irradiated surface due to the photoelectric effect. Thus, a radiation-generated short-lived plasma is formed in the volume above mirror surfaces, which may be used to clean these surfaces in-line [23].

At $9 \mathrm{~Pa}$ of $\mathrm{H}_{2}$ pressure, the plasma potential of EUV-induced plasma is only few eV, the ion density is just $\mathrm{n}_{\mathrm{i}} \sim 10^{7} \mathrm{~cm}^{-3}$ and plasma is relatively cold $\left(\mathrm{T}_{\mathrm{e}} \sim 1-2 \mathrm{eV}\right)$. The estimated electron density is $3-4 \cdot 10^{7}$ $\mathrm{cm}^{-3}$ and drops after 3 microseconds. The ion's energy is on the order of the binding energy of atoms within the contamination layer.

In comparison, the ion density is two orders of magnitude higher in plasma reactors. Taking into account the low density and ion energy, the efficiency of radiation driven plasma cleaning must be checked experimentally. Although the plasma potential is low, the ion energy can be increased through the use of biasing (see following chapters). However, the density and short lifetime of the plasma are given by EUVL operating conditions, which cannot be easily altered.

In order to understand the physics and chemistry of the surface plasma-cleaning process, experimental work was performed using a unique set of tools. Chapter 2 contains a description of the experimental setup to generate and characterize radiationgenerated plasmas, and plasma-surface interactions. The radiation 
source, with intensity and pulse duration relevant to EUVL, and diagnostic tools for experimental measurements, are described in detail.

To study elementary plasma processes, a time-resolved Langmuir probe system was applied. In Chapter 3, the perturbations that the probe introduces into plasma are investigated using both experimental data and a spatio-temporal numerical model of the plasma [24]. We show that, by combining the Langmuir probe measurements with the numerical model, the plasma parameters in the absence of the probe can be determined. The relevancy of a modeling approach with experimental data, compared to the direct extraction of any plasma parameters from the probe curves is discussed.

The study of carbon removal processes under EUV exposure is presented in Chapter 4. It is shown that carbon can be efficiently etched from multilayer mirrors in a low-temperature low-pressure plasma. Earlier experiments had shown that the efficiency of removing amorphous carbon from the MLM surface using plasmas was found to be much higher than that of atomic hydrogen cleaning [17]. Moreover, in presence of EUV radiation plasma cleaning is even more effective. A new reactive-ion process is presented and physical mechanisms for EUV plasma cleaning are discussed.

Within the experiments from chapter 4, magnetron deposited carbon was used as a model for EUV-induced carbon growth. However, the characteristics of a carbon film depend on the film deposition conditions. In practice, this means that the optics positioned close to the radiation source could be coated with a significantly denser carbon film, due to the presence of a broad spectrum of high energy photons and ions. 
Chapter 5 reports the experimental study of carbon-based films, deposited on the collector mirror of an EUV source. X-ray photoelectron spectroscopy (XPS), Raman spectroscopy (RS), energydispersive X-ray (EDX) spectroscopic analysis, transmission electron microscopy (TEM), and other analysis of the film show that the contaminant has DLC characteristics.

Furthermore, a plasma assisted cleaning experiment was carried out. It was found that hydrogen ions are less effective for such DLC contamination removal, compared to amorphous carbon. Hence, it is necessary to know how the phase of carbon may vary, depending on the growth conditions, to choose the optimal MLM maintenance strategy.

In the sixth chapter, an analysis of the main mechanisms for oxide reduction is presented. The potential for balancing oxidation and reduction through the use of EUV-generated ions and radicals is evaluated. Specifically, the possibility for in-line cleaning, via lowpressure hydrogen plasma is considered.

As one can see, the practical problem of maintaining the performance of EUV optics raises a number of physics questions that can be more adequately addressed using model systems. 


\section{References}

1. Banine V Y, Koshelev K N, Swinkels G H P M 2011 J. Phys. D: Appl. Phys. 44253001.

2. Hansson B 2003 Laser-Plasma Sources for Extreme-Ultraviolet Lithography Doctoral Thesis Stockholm Sweden

3. Graef W 2009 Time dependent collisional radiative model of an extreme ultraviolet driven plasma Doctoral Thesis Eindhoven University of Technology Netherlands

4. Lin B J 2006 J. Microlith., Microfab., Microsyst. 533005.

5. Louis E, Yakshin A E, Tsarfati T, Bijkerk F 2011 Progress in Surf. Science $86255-294$.

6. Bosgra J 2013 Interlayer thermodynamics in nanoscale layered structures for reflection of EUV radiation, Doctoral Thesis University of Twente Netherlands

7. Louis E 2012 Physics and technology development of multilayer EUV reflective optics Doctoral Thesis University of Twente Netherlands

8. Juequan C 2011 Characterization of EUV induced contamination on multilayer optics Doctoral Thesis University of Twente Netherlands

9. Oizumi H, Izumi A, Motai K, Nishiyama I, and Namiki A, 2007 Jap. J App. Phys Part 2-Letters \& Express Letters 46 L633-L635.

10. Silverman PJ 2005 J. Microlith., Microfab., Microsyst. 4(1) 011006.

11. Seisyan R P 2005 Techn. Phys. 50-5 535-545.

12. Koster N, Mertens B, Jansen R, van de Runstraat A, Stietz F, Wedowski M, Meiling H, Klein R, Gottwald A, Scholze F, Visser M, Kurt R, Zalm P, Louis E, and Yakshin A 2002 Microelectron. Eng. 61-2 65-76.

13. Bienert M, Göhnemeier A, Natt O, Lowisch M 2009 J. Micro/Nanolith. 8-4 041509.

14. Banine V, Benschop J 2003 Proc. of SPIE 54017861.

15. Hill S B, Faradzhev N S, Tarrio C, Lucatorto $T$ B, Madey $T$ E, Yakshinskiy B V, Loginova E, Yulin S 2008 Proc. of SPIE $6921692117-$ 1.

16. Hollenshead J, Klebanoff L 2006 J. Vac. Sci. Technol. B 24, 118. 
17. Braginsky O V, Kovalev A S, Lopaev D V, Malykhin E M, Rakhimova T V, Rakhimov A T, Vasilieva A N, Zyryanov S M, Koshelev K N, Krivtsun V M, M van Kampen and Glushkov D 2012 J App. Phys. 111093304.

18. Zyryanov S M, Kovalev A S, Lopaev D V, Malykhin E M,Rakhimov A T, Rakhimova T V, Koshelev K N, and Krivtsun V M 2011 Plasma Phys. Rep. 37-10 881-889.

19. Motai K, Oizumi H, Miyagaki S, Nishiyama I, Izumi A, Ueno T, and Namiki A, 2008 Thin Solid Films 516 839-843.

20. Graham S, Charles A S, Clift W M, Leonard E K, and Sasa B 2003 SPIE, 5037 460-469.

21. Tsarfati T, Zoethout E, R W E van de Kruijs, and Bijkerk F 2009 Surf. Sci 603 2594-2599.

22. Ugur D, Storm A J, Verberk R, Brouwer J C, Sloof W G 2013 Microelectron. Eng. 110 60-65.

23. Astakhov D I, Goedheer W J, Lee C J, Ivanov V V, Krivtsun V M, Koshelev K N, Lopaev D V, van der Horst R M, Beckers J, Osorio E A and Bijkerk F 2016 J. Phys. D: Appl. Phys. 4929.

24. Astakhov D 2016 Numerical study of extreme-ultra-violet generated plasmas in hydrogen Doctoral Thesis University of Twente Netherlands 


\title{
2. Extreme ultraviolet source and ultra high vacuum chamber for studying EUV-induced processes
}

\begin{abstract}
An experimental setup that directly reproduces Extreme UVlithography relevant conditions for detailed component exposure tests is described. The EUV setup includes a pulsed plasma radiation source, operating at $13.5 \mathrm{~nm}$; a debris mitigation system; collection and filtering optics; and an UHV experimental chamber, equipped with optical and plasma diagnostics. The first results, identifying the physical parameters and evolution of EUV-induced plasmas, are presented. Finally, the applicability and accuracy of the in situ diagnostics is briefly discussed.
\end{abstract}

\section{Introduction}

The next generation of photolithography, extreme ultraviolet (EUV) lithography, is expected to be required at the $13.5 \mathrm{~nm}$ node $[1,2]$. The ionizing photon flux, and vacuum requirements create a challenging operating environment. One of the most important requirements is optics purity with most of the optical elements expected to last for the lifetime of the photolithography tool. The basic optical element in EUV lithography (EUVL) is the multilayer mirror (MLM). MLMs consist of approximately 50 bi-layers of $\mathrm{Mo} / \mathrm{Si}$ that are $6.7 \mathrm{~nm}$ thick, with the uppermost layer being a protective layer that is for instance $2 \mathrm{~nm}$ thick. A thorough review of these mirrors is given in [3]. Multilayer mirrors in EUVL are expensive, hightechnology items, making it desirable to extend their useful lifetime as much as possible. 
Previous research has demonstrated that MLMs may lose their reflectivity due to their surfaces becoming contaminated with amorphous carbon or surface oxidation, induced by intense EUV radiation [4,5]. One solution that is currently under investigation is using EUV-induced plasma for in-line cleaning [6]. Experiments have shown that carbon etching can be achieved under certain conditions, but the physics and chemistry of the etching process is still not fully understood. One of the reasons for this lack of understanding is that the characteristics of the EUV radiation-induced plasma are poorly known.

In this article we present an experimental setup that allows EUVinduced processes to be studied. These processes include carbon contamination, surface oxidation, and plasma cleaning, as well as plasma-assisted processes like ion sputtering and blistering [7, 8, 9]. Carbon contamination was previously studied using an electron beam as a proxy for EUV [10]. However, electron beam irradiation is not fully analogous to the situation found in an EUV tool, where high energy photons play a significant role in plasma chemistry. This problem can be solved by using synchrotron radiation [11,12], but such experiments are impossible in ordinary laboratories. Currently there are a number of laboratory EUV sources, based on vacuum discharge in xenon or tin. The former sources are more common, but the latter have much greater conversion efficiency (up to $2 \%$ in tin compared to $0.5-0.7 \%$ in xenon) [1].

For the study of elementary processes in the EUV-induced plasma, the apparatus must be equipped with the appropriate diagnostics. Recent direct studies of the EUV-induced plasma were successfully carried out by using a method called microwave cavity resonance spectroscopy [13], but in this case, surface processes, such as secondary electron emission and surface plasma formation cannot 
be studied. However, EUV induced secondary electrons, which escape from the surface of the topmost or capping layer, are able to trigger surface reactions, possibly with an even higher efficiency than direct EUV photoionization. These secondary electrons produce additional ionization in the gas above the optic's surface, leading to a plasma sheath with an increased degree of ionization. Such a nearsurface layer with enhanced electron density may strongly influence the surface photo- and plasma-chemistry, including practical cleaning schemes.

As we can see, a lot of physical phenomena (photoionization, secondary electron emission, ion plasma production etc.) contribute to the processes above the surface under EUV radiation. At the moment it is not clear which of them are more efficient at cleaning surfaces, despite the fact that the influence of EUV light on cleaning has been shown experimentally in ref [6]. However, near-surface elementary processes, occurring in the EUV-induced plasma, are poorly understood. Depending on the type of a gas and pressure in the volume above the surface, different types of ions and radicals (cleaning agents) predominate at different times. The formation of different cleaning agents strongly depends on the speed of plasma transport and the decay time of the plasma. Therefore, an experimental setup for the study of time evolution of the plasma parameters is required to understand EUV-induced surface plasma processing.

An experimental setup, that is able to characterize low-density EUVinduced plasmas using a time-resolved Langmuir probe system is presented in this work. Below, the radiation source, with intensity and pulse duration relevant to EUVL, and diagnostic tools for experimental measurements, are described in detail. 
The EUV source, based on a discharge-produced plasma in tin vapor, irradiates samples in a clean vacuum chamber. The latter is designed for the investigation of surface and volume physical and chemical processes, induced or assisted by EUV. The benefits of this setup are high EUV power density, technical simplicity, and good control over the background gas and electric fields in the clean cylindrically symmetrical chamber. The addition of surface biasing and cylindrically symmetric fields makes the setup amenable to numerical calculations.

We also describe the diagnostic equipment for studying the EUVinduced plasma. Such plasma is difficult to study because of the low density (the electron density hardly exceeds $10^{7}-10^{10} \mathrm{~cm}^{-3}$ ). In such cases optical diagnostics are inefficient, while previous work suggested that Langmuir probes would be infeasible [14]. Here, however, we show that Langmuir probes for measuring the plasma density and temperature are effective. We succeeded in obtaining the temporal dynamics of the electron temperature and density in a low-density EUV-induced plasma. These measurements are presented at the end of the chapter.

\subsection{Setup}

The installation shown in figure 1 (PROTO 2 setup) consists of several main parts. The first part is the discharge vacuum chamber, equipped with spouts for the entry of laser radiation and the installation of diagnostic sensors. In addition, the chamber has four flanges available for optical observations of the discharge. The chamber pumping system consists of two pumps, capable of 1000 $\mathrm{l} / \mathrm{s}$, resulting in an operating pressure of $10^{-3} \mathrm{~Pa}$. 
The lower part of this chamber contains the source of EUV radiation - highly ionized tin plasma - initiated by a laser pulse in the discharge gap (3-4 $\mathrm{mm}$ ) between the anode and cathode. The anode and liquid tin-coated cathode rotate synchronously at a frequency of up to $30 \mathrm{~Hz}$ and operate at a potential difference up to $5 \mathrm{kV}$. One load of tin is sufficient to maintain continuous operation for 17 hours, which is approximately 100 MShots.

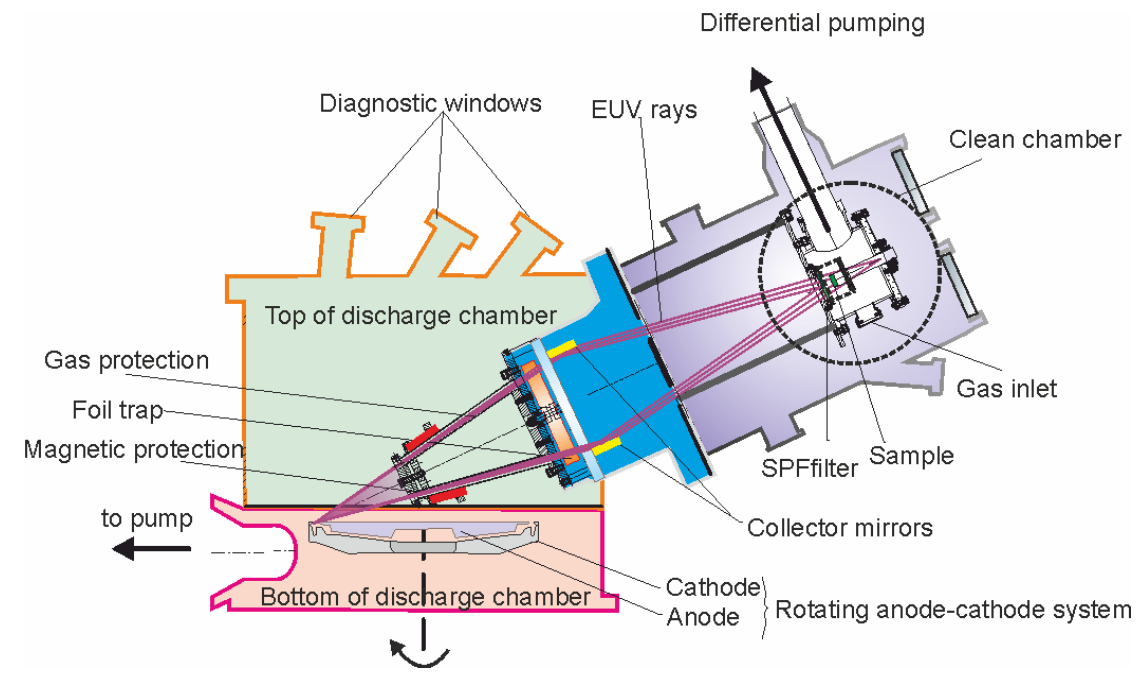

Figure. 1. EUV source, based on discharge-produced plasma in tin vapor, with a UHV vacuum chamber for the investigation of surface and volume physical and chemical processes, induced or assisted by EUV.

The tin is evaporated from the cathode using an Nd:YAG laser that generates pulses with a duration of $50 \mathrm{~ns}$, and energies up to $37 \mathrm{~mJ}$, at a pulse repetition rate of $1.6 \mathrm{kHz}$. The average laser radiation power incident on the cathode is approximately 56 watts. 
The upper part of the discharge chamber contains collecting optics and the debris mitigation system. The collection optics and debris mitigation system are mounted in an independent housing, that is attached to the side-wall of the upper discharge chamber at an angle of $30^{\circ}$ relative to the plane of the electrodes. The radiation is introduced into the chamber using a metal conical light guide with $10 \mathrm{~mm}$ gap,

To prevent neutral tin atoms from entering the guide, hydrogen flows in the opposite direction with a flow rate of $0.001 \mathrm{l} / \mathrm{s}$. Along the guide, there are six SmCo magnets, that create a field of $0.5 \mathrm{~T}$ in the guiding structure. The magnetic field extends over a length of $10 \mathrm{~cm}$, and protects the collector mirrors from tin ions with energies up to $100 \mathrm{KeV}$. Finally, a rotating foil trap is used to protect the collection optics from droplets of tin created by the discharge [15]. The foil trap consists of 150 molybdenum foil blades, $20 \mathrm{~mm}$ long, on a disk with a diameter of $150 \mathrm{~mm}$. The trap rotates with the frequency of $100 \mathrm{~Hz}$ and is located directly in front of the light collector. To pass through the foil trap, particles are required to have a speed of $500 \mathrm{~m} / \mathrm{s}$. Combined with the magnetic field trap, only Z-pinch ions above $150 \mathrm{keV}$ are incident on the collector optics.

The light collector consists of grazing incidence cylindrical mirrors that are coaxially mounted. The mirror substrates are made from sital, and shaped by deep grinding and polishing. The radius of the mirrors is $75 \mathrm{~mm}$, and the height is $45 \mathrm{~mm}$. The focal distance of the collector is $480 \mathrm{~mm}$. The mirror coating consists of a $100 \mathrm{~nm}$ thick layer of Mo, deposited by magnetron sputtering and has an EUV reflectivity of $83 \%$ with surface roughness $\sim 7 \mathrm{~nm}$.

To prevent carbon from accumulating on the mirror surface [4], the mirrors are biased at $150 \mathrm{~V}$ [6]. This bias voltage provides a flux of 
low energy hydrogen ions to the surface, which act as a cleaning agent.

\subsubsection{UHV chamber}

EUV radiation from the tin plasma is refocused by the collector optics at the sample location in the clean chamber. The UHV chamber is separated from the main volume by a $200 \mathrm{~nm}$ thick $\mathrm{Zr}$ filter, mounted on a nickel grid [16]. The spectral purity filter (SPF) is opaque from the deep UV to visible range and transmits EUV radiation at a wavelength of the $13.5 \mathrm{~nm}$. Radiation spectra from Zpinch discharge with and without SPF in range $10-25 \mathrm{~nm}$ is presented in figure 2.

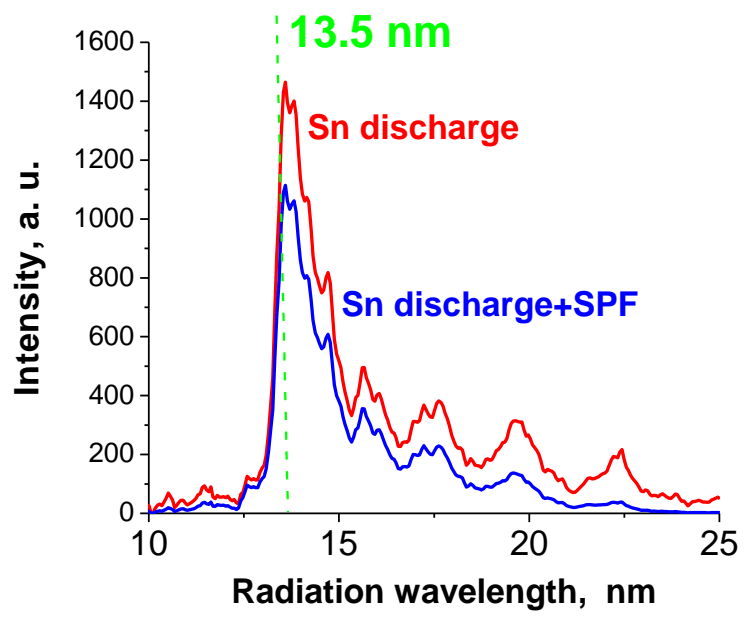

Figure 2. Radiation spectra from Z-pinch discharge in tin vapor with and without spectral purity filter (SPF) in the range of 10-25 $\mathrm{nm}$.

The filter is mounted to the chamber using indium seals, and provides sufficient physical strength to allow isolation even in the 
presence of large differential pressures. This allows the pressure in the clean chamber to be varied from $10^{-6} \mathrm{~Pa}$ to $100 \mathrm{~Pa}$. The pressure difference between the clean chamber and the discharge chamber is limited by the tensile strength of the SPF as well as its sealing quality. The maximum difference is determined by the gas leaking from the clean chamber, which should not create a pressure greater than 0.06 $\mathrm{Pa}$ in the discharge. Pressures higher than that have an adverse effect on the discharge and significantly lower the EUV power.

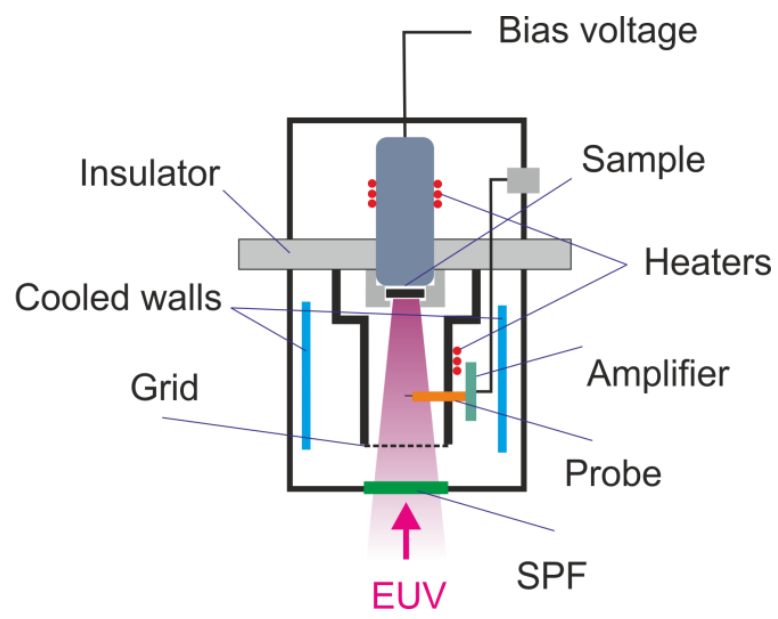

Figure 3. Schematic view of the 'clean' chamber. Clean chamber is equipped with heating, cooling-including a cryogenic dosing system-differential pumping, and sample mounts with the provision for biasing samples. The bias potential of 200 to -200 volts can be applied to the sample. A copper cylinder serves as an anode and is mounted above the sample.

The UHV chamber (see figure 3 ) is equipped with heating, coolingincluding a cryogenic dosing system-differential pumping, and sample mounts with the provision for biasing samples. 
Table 1. Basic parameters of the PROTO2 tin EUV source with exposure chamber.

\begin{tabular}{|c|c|}
\hline \multicolumn{2}{|c|}{ Main parameters of PROTO 2 experimental setup } \\
\hline Maximum source power consumption & $3 \mathrm{~kW}$ \\
\hline Mean electric energy of discharge output & $1.85 \mathrm{~J} /$ pulse. \\
\hline $\begin{array}{l}\text { In-band discharge to optical conversion ( } 2 \pi \text { solid } \\
\qquad \text { angle) }\end{array}$ & $1.5 \%$ \\
\hline Pulse repetition rate & $1.6 \mathrm{kHz}$ \\
\hline Pulse duration of EUV radiation & $100 \mathrm{~ns}$ \\
\hline EUV intensity in focus spot $D=6 \mathrm{~mm}$ without SPF & $\begin{array}{c}0,75 \mathrm{~W} / \mathrm{cm}^{2}\left(4.910^{-4}\right. \\
\left.\mathrm{J} / \mathrm{cm}^{2} / \text { pulse }\right)\end{array}$ \\
\hline EUV intensity in focus spot $D=6 \mathrm{~mm}$ with SPF & $\begin{array}{c}0,13 \mathrm{~W} / \mathrm{cm}^{2}\left(0.8510^{-4}\right. \\
\left.\mathrm{J} / \mathrm{cm}^{2} / \text { pulse }\right)\end{array}$ \\
\hline \multicolumn{2}{|c|}{ Exposure clean chamber and probe system parameters } \\
\hline Chamber temperature range & from $-100^{\circ} \mathrm{C}$ to $+150^{\circ} \mathrm{C}$ \\
\hline Exposure chamber pressure range & from $10^{-6} \mathrm{~Pa}$ to $100 \mathrm{~Pa}$ \\
\hline Sample bias voltage range & from $-200 V$ to $200 V$ \\
\hline Langmuir probe bias voltage range & from $-190 \mathrm{~V}$ to $190 \mathrm{~V}$ \\
\hline
\end{tabular}


The energy characteristics of EUV radiation of discharge plasma were measured using the pin diode (AXUV 100, IRD Inc.), shielded by an SPF filter. The power of EUV radiation, introduced to the 'clean' chamber, was measured using a specially designed and calibrated calorimeter, based on an AD590 temperature sensor.

The basic parameters of the EUV source and sample chamber are provided in the table 1.

\subsection{Study of the EUV-induced plasma.}

To characterise the low-density EUV-induced plasma that forms in the clean chamber, a system of Langmuir probes and low noise electronics was installed.

\subsubsection{Probe measurement scheme.}

Figure 4 provides a full scheme of probe characteristics measurement of the EUV-induced plasma. The plasma is formed between the two electrodes. The first electrode, which is irradiated with EUV, is a copper disc with a mount for 1 " (25 mm) wafers. This electrode can be electrically biased in the range of -200 to +200 volt. In our experiments, only negative bias voltages were applied, so this electrode served as a cathode. The other electrode, used as an anode, is a hollow copper cylinder with a height of $40 \mathrm{~mm}$ and an inner diameter of $32 \mathrm{~mm}$. The two electrodes are mounted coaxially with the EUV beam, as shown in figure 4. From the SPF side, the cathode is covered with a metal grid with a $1.5 \mathrm{~mm}$ spacing, which prevents the electrons emitted from SPF from entering the interelectrode gap. The cathode and the anode are connected in an electric circuit, allowing the current flowing in the gap to be measured. There are three mounting holes for Langmuir probes 
(figure shows only one probe) along the axis of the cylinder of the anode. The distances from the probe locations to the sample surface are 12,20 , and $28 \mathrm{~mm}$. Since each probe, in principle, disturbs the plasma, all measurements are performed with a single probe installed at a chosen location to minimize the probe influence while still obtaining an accurate measurement of the spatial dependence of the plasma. The probe consists of a steel wire of $0.5 \mathrm{~mm}$ diameter in a ceramic shell that leaves a $5 \mathrm{~mm}$ length of wire exposed to the plasma.

Before entering the chamber, gases are passes through a liquid nitrogen trap to get rid of impurities. The temperature of the sample and the anode is measured with a thermocouple and are kept at a constant temperature through active cooling and heating. Heating is provided by cement resistor heaters. Cooling is achieved using liquid nitrogen. The electrical connections pass through a sealed iron housing that provides screening from external crosstalk. In case of experiments, that described in this chapter the clean chamber was filled with hydrogen.

A low-noise amplifier circuit was used to acquire the probe signals. It consists of two amplifier cascades. The current signal of the probe is converted to a voltage signal by a transimpedance amplifier, based on an OPA656 operational amplifier. The non-inverted input of the amplifier, as well as the power supply terminals, have the same potential as the output of the high-voltage amplifier that generates the bias voltage. Due to the feedback of the transimpedance amplifier, the inverting input, to which the probe is connected, has the same voltage. To minimize crosstalk, this amplifier is mounted in the clean chamber next to the probe. It must be mentioned that, under typical experimental high vacuum conditions, no fabricreinforced laminate may be used because it releases gases. Thus, all 
the boards placed in the clean chamber are ceramic-based. The signal from the transimpedance amplifier is transferred to a differential amplifier that removes the common-mode interference from the signal and amplifies the signal by a factor of five.

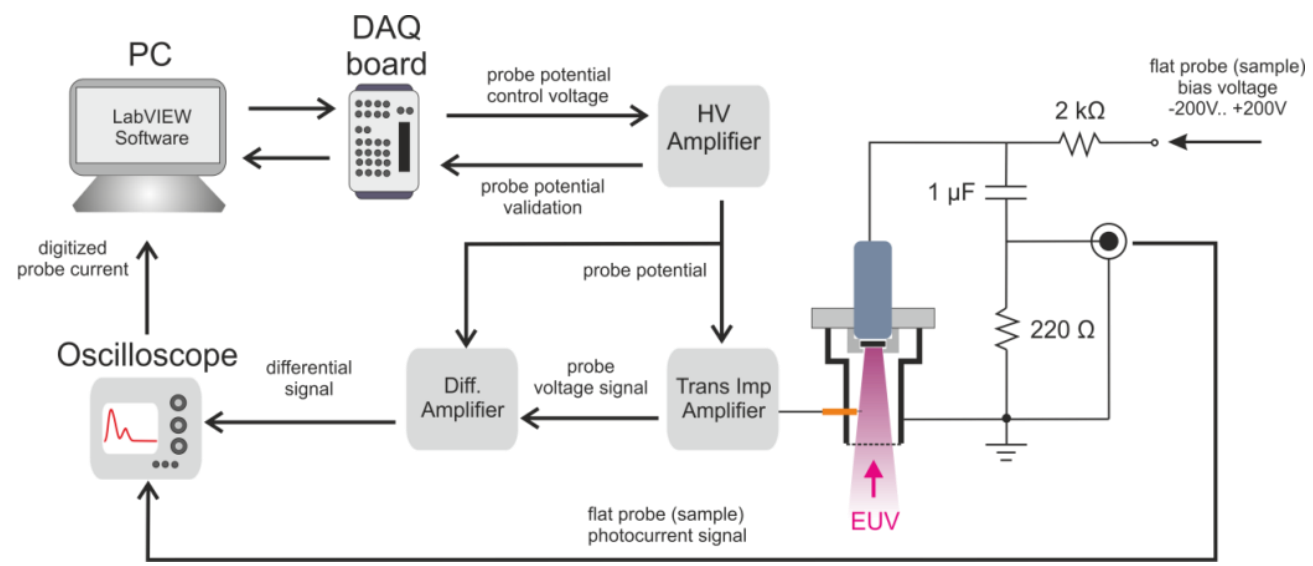

Figure 4. Block diagram of hardware and software that were used for EUV-induced plasma dynamics studying.

The resulting signal is recorded by an oscilloscope (Tektronix DPO 3014), which, to reduce noise, operates in averaging mode. When averaging 512 oscilloscope records, a noise amplitude of approximately $10 \mathrm{nA}$ is reached, which is low enough to measure the currents that correspond to an ion density on the order of $10^{6} \mathrm{~cm}^{-3}$.

Figure 4 also provides a scheme for measuring the current through the sample, which behaves as if it is a flat probe. The focus of the collector contains a sample holder (a large copper electrode with an attachment for inch-size silicon substrates). A bias potential of 200 to -200 volts can be applied to the sample, using an external power source. A copper cylinder, which serves as an anode, is mounted 
above the holder. The holder and the anode are included in the circuit provided in the figure 4. This circuit allows real-time monitoring of the current leaving the sample, when exposed to EUV radiation.

\subsection{Experimental results}

A straightforward way to characterize the EUV-induced plasma is to measure the current in the inter-electrode gap. We refer to this as a "photocurrent" since a significant contribution to it is made by the photoelectric effect from the sample.

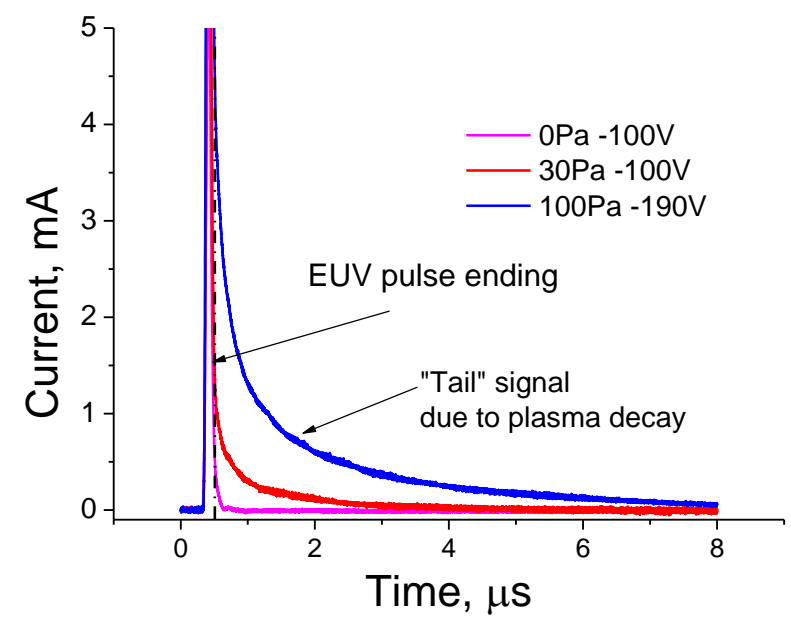

Figure 5. The photocurrent, measured at different gas pressures and bias voltages. The tail is due to the ion current and illustrates the lifetime of the plasma formed during photo-ionization, and the ionization of the gas by photoelectrons close to the sample.

The photocurrent measurements of the sample (copper disk) were carried out at different hydrogen gas pressures in the clean chamber. The pressure was fixed for each of the individual 
measurements. When a chosen bias value was reached, the time dependence of the current in the circuit between the sample and the cylindrical anode was recorded. In a vacuum this current is characterized by a duration comparable to the duration of EUV pulse radiation, i.e., $\sim 100$ ns.

In the presence of a gas, the signal has a long plasma tail (see figure 5), fading in the 4-6 $\mu$ s after the start of acquisition (and long after the end of the EUV pulse). The slow tail is due to the ion currentillustrating the lifetime of the plasma formed during photoionization-as well as due to the ionization of the gas by photoelectrons above the sample.

The integration of the slowly decaying tail provides a charge that can be used to determine the ion dose incident on the sample. Moreover, assuming that plasma is localized in the volume of the cylindrical anode, it is possible to provide an approximate estimate of ion density. For this purpose, the pulse is integrated from the end of the EUV radiation pulse (moment $t_{0}$ ):

$$
n_{e}=n_{i} \approx \frac{2}{e V_{a}} \int_{t_{0}}^{t_{\max }} I_{P}(t) d t
$$

where $V_{a}$ is the volume of the cylinder of the anode, $I_{p}$ is the current of the sample at gas pressure $P$, e is the electron charge, $t_{0}$ is the time of the end of the EUV pulse. The multiplier 2 takes into account the fact that the charge is collected on the sample from the nearelectrode layer and not from the entire volume.

Figure 6 shows the charge-bias characteristics for a sample exposed to an EUV-induced hydrogen plasma. In this case, based on the curve 
in figure 6 , the plasma ion density is estimated to be $n_{i} \sim 10^{7} \mathrm{~cm}^{-3}$. Thus, as expected, the density of the plasma is low.

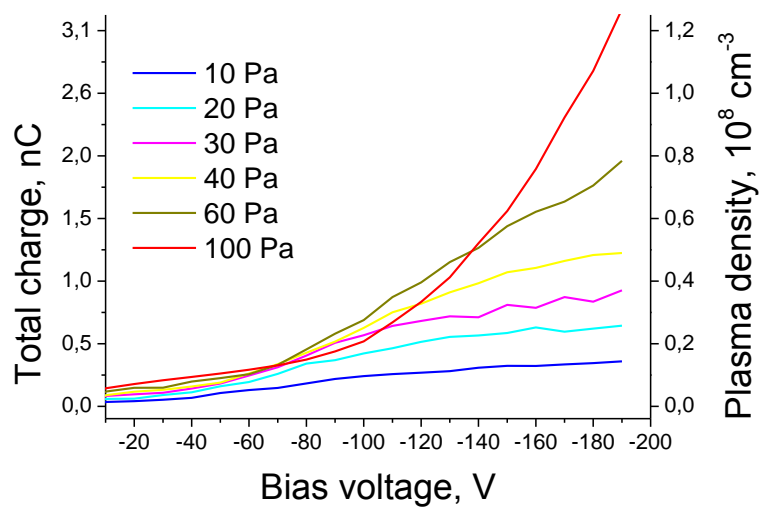

Figure 6. The charge-bias characteristics for a sample exposed to an EUV-induced hydrogen plasma at different gas pressures.

\subsection{Probe measurements}

In each measurement, the pressure, and the offset voltage supplied to the sample, over which the probe is placed, remain unchanged. Measurements were taken at relatively high pressure $(20 \mathrm{~Pa})$, and high sample potential ( $20 \mathrm{~V}$ ) to limit the effect of the Langmuir probe on the plasma. The potential of the probe is controllably varied, and the current pulse from the probe was acquired for each probe potential. Cutting the current data at a constant time results in a I-V characteristics. Figure 7 shows the $I-V$ curve for a number of different times.

The Volt-Ampere characteristics of the EUV induced plasma, shown in figure 7, are quite typical for a low-density, low-temperature plasma. The floating potential of the probe, $V_{f}$, varied within a range 
of $\pm 2 \mathrm{~V}$. The electron contribution to the current appears abruptly, which indicates that the plasma is, as expected, relatively cold $\left(T_{e} \sim 1\right.$ $2 \mathrm{eV})$.

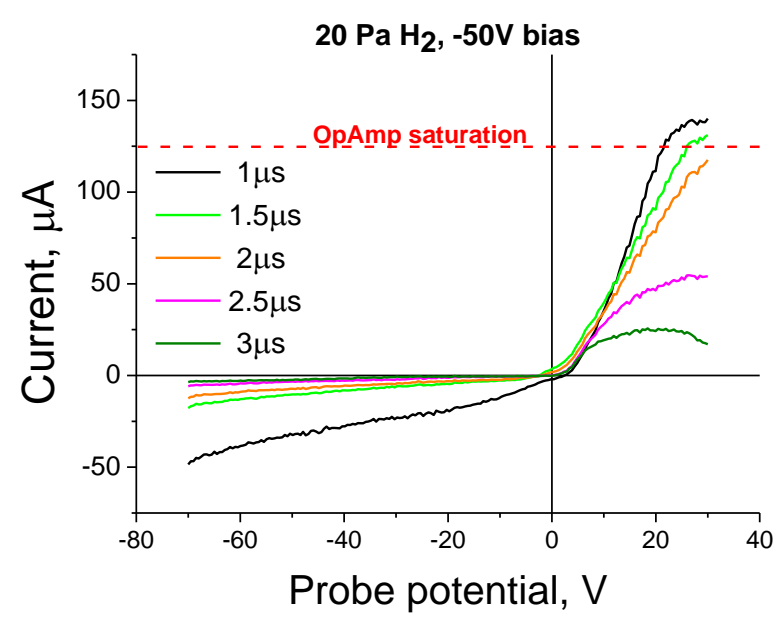

Figure 7. Volt-Ampere characteristics of the EUV induced plasma measured at the different times for a gas pressure of $20 \mathrm{~Pa}$, and a sample bias of $50 \mathrm{~V}$. $\mathrm{T}_{e}$ can be estimated from the slope of the near-exponentially decreasing portion of the curve. The electron contribution to the current appears abruptly, which indicates that the plasma is, as expected, relatively cold.

To estimate the density of electrons, Laframboise's theory of charge collection $[17,18]$ was applied (see figure 8). The estimated electron density was $3-4 \cdot 10^{-7} \mathrm{~cm}^{-3}$, which is in good agreement with the estimate based on the charge-bias characteristic made above. Taking into account the quasineutrality of the plasma, we know the density of ions at the same time, which is the density of the active cleaning species after EUV pulse. As we can see in figure 8 immediately after the radiation pulse, the plasma temperature is relatively high due to hot photoelectrons. After first two microseconds, electrons cool to a temperature of about 1-1.5 eV, which is typical for low-temperature 
plasmas. This temperature is maintained in the plasma for up to 4 microseconds, or during the entire plasma lifetime. To study plasma dynamics during the EUV pulse, it is necessary to take into account the emission from the surface of the probe, which we have not yet studied.

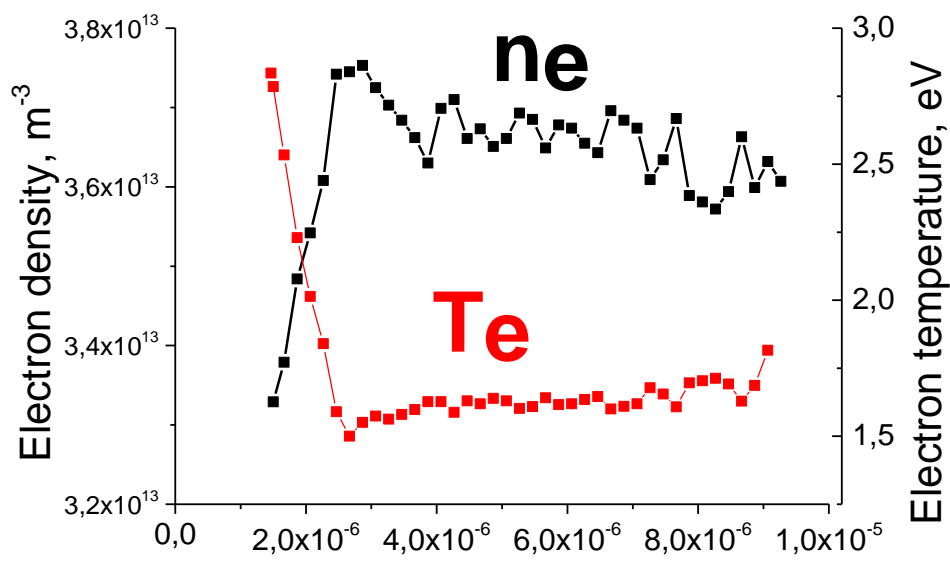

Time,s

Figure 8. The plasma density $n_{e}$ and electron temperature $T_{e}$ of EUV-induced plasma at $20 \mathrm{~Pa}$ of hydrogen and a sample bias of $50 \mathrm{~V}$ determined using the methods of the semi-logarithmic electron current plot and Laframboise's theory of charge accumulation. The estimated electron density $3-4 \cdot 10^{7} \mathrm{~cm}^{-3}$ is in good agreement with the estimate based on the charge-bias characteristic.

\subsection{Conclusion}

An experimental setup for the study of EUV-induced plasma directly interacting with surfaces at Extreme UV-lithography relevant conditions has been described. The installation is equipped with a 
system of Langmuir probes that are able of measuring the temporal and spatial development of very low density plasmas. Since the surface chemistry of MLMs is often driven by plasma-induced process, knowledge of the plasma properties is critical to understanding surface chemistry. The experimental setup described here is expected to allow further fundamental studies of surface physics and chemistry, supported by accurate plasma characterization.

The determined plasma characteristics include: the temperature of electrons, and the density of plasma. Measurements can be performed over a wide range of pressures and cathode biases. The pressure in the clean chamber can be varied from $10^{-6} \mathrm{~Pa}$ to $100 \mathrm{~Pa}$. The cathode can be electrically biased in the range of -200 to +200 volt. It is also possible to study the temporal dynamics of plasmas after the EUV pulse.

Our initial results show that the characteristic development of a lowtemperature plasma, excited by pulsed EUV radiation, are in good agreement with preliminary estimates of the plasma parameters. The experiments show that the electron density for EUV-induced plasma increases from the moment of the beginning of the pulse and lasts a few microseconds. In these measurements the density of all gas pressures studied never exceeds $5 \cdot 10^{8} \mathrm{~cm}^{-3}$ and drops after 3 microseconds. 


\section{References}

1. Bakshi V 2006 EUV sources for lithography. (SPIE press).

2. Silverman P J. 2005 Journal of Micro/Nanolithography, MEMS, and MOEMS 4.1 011006-011006.

3. Louis E, Yakshin A E, Tsarfati T, Bijkerk F 2011 Progress in Surf. Science 86255 -294.

4. Chen J Q, Louis E, Lee C J, Wormeester H, Kunze R, Schmidt H, Schneider D, Moors R, van Schaik W, Lubomska M, and Bijkerk F 2009 Optics Express 17 16969-16979.

5. Hiroaki O et al. 2007 Jap. J App. Phys 46 L633-L635.

6. Dolgov A., et al. 2014 J. Phys. D: Appl. Phys 47065205.

7. Allain J P, Hassanein A, Allain M M C, Heuser B J, Nieto M et al 2006 Nuclear Instruments and Methods in Physics Research Section B: Beam Interactions with Materials and Atoms, 242 520-522.

8. Kuznetsov A S, Gleeson M A, and Bijkerk F, 2012 J. Phys. Condens. Matter 24052203.

9. Kuznetsov A S, Gleeson M A, van de Kruijs R W E and Bijkerk F 2011 Proc. SPIE 8077807713.

10. Kurt R, van Beek M, Crombeen C, Zalm P, Tamminga Y 2002 Proc. SPIE, 46880277.

11. Socol Y, Kulipanov G N, Matveenko A N, Shevchenko O A, and Vinokurov N A 2011 Phys. Rev. ST Accel. Beams 14040702.

12. Al-Ajlony A, Kanjilal A, Sivanandan S H and Hassanein A 2012 J. Vac. Sci. Technol. B 30041603.

13. van der Horst R M, Beckers J, Nijdam S, Kroesen G M W 014 J. Phys. D: Appl. Phys. 47302001.

14. van der Velden H L 2008 Radiation generated plasmas Doctoral Thesis Eindhoven University of Technology Netherlands.

15. Shmaenok L A, de Bruijn C C, Fledderus H F, Stuik R, Schmidt A A et al 1998 Proc. SPIE 333190.

16. Chkhalo N I at el.2012 J. Micro/Nanolith. MEMS MOEMS 11021115.

17. Chen F F.2003 IEEE-ICOPS Meeting, Jeju, Korea.

18. Laframboise J G 1966 Univ. Toronto Aerospace Studies Report 11. 


\section{Numerical simulations based on probe measurements in EUV-induced hydrogen plasma}

\section{Abstract}

We use a two-dimensional Particle-in-Cell model with Monte Carlo collisions to study the plasma induced in hydrogen by short pulses of extreme ultraviolet (EUV) radiation at wavelengths in the range 10-20 nm with a pulse duration of about $40 \mathrm{~ns}$ (FWHM). This plasma is formed via both photoionization by high-energy EUV photons and secondary photoelectrons emitted from the hydrogen molecules and the irradiated surface. The latter process can be enhanced by an external electric field that accelerates the electrons. In order to establish a base for our model so as to obtain accurate results, we record a temporally-resolved series of current-voltage characteristics for a small probing electrode, inserted into EUV-induced hydrogen plasma. We then resort to simulating this plasma in the same geometry, including the probe, and validate the model by matching its predictions to the experimentally measured dynamics of the probe current-voltage curves. Having validated the model this way, we use the model as an independent instrument, capable of obtaining the spatio-temporal picture of EUV-induced plasma evolution. We use this instrument to study plasma formation during the EUV pulse and point out the processes that take part in forming the plasma, such as impact ionization and direct ionization by the EUV photons.

\section{Introduction}

Here we use the term "EUV-induced plasma" to denote a plasma that is formed in a low pressure (a few ten of pascal) background gas by the ionizing action of extreme ultraviolet (EUV) radiation. EUV 
radiation is usually classed as radiation with wavelengths in the 5-40 $\mathrm{nm}$ range, which corresponds to photon energies from about 30 to $250 \mathrm{eV}$ [1]. Due to such high energies, EUV photons allow direct photoionization of the background gas, generating high energy electrons, which are also capable of ionization. At the same time, EUV photons can generate energetic photoelectrons from any irradiated surface due to the photoelectric effect. Thus, a radiationgenerated plasma can be formed both by direct photoionization and by impact ionization from secondary electrons. External electric fields can enhance impact ionization, as the, initially slow, secondary electrons can gain sufficient energy to ionize atoms and molecules.

Studies of EUV-induced plasmas are of direct relevance for understanding and controlling the operation conditions of multilayer mirrors serving as EUV optics [2]. An important example of the application of EUV optics is extreme ultraviolet lithography (EUVL). EUVL makes use of $13.5 \mathrm{~nm}$ radiation, emitted by a pulsed hot highdensity tin plasma [3]. The optical column of an EUVL machine operates in a vacuum chamber with a low pressure hydrogen atmosphere. Because of this, an EUV-induced hydrogen plasma is formed within the vacuum chamber. This plasma usually has a low density $\left(10^{7}-10^{9} \mathrm{~cm}^{-3}\right)$ and a short lifetime (down to a few $\left.\mu \mathrm{s}\right)[4,5,6]$.

Being in contact with the EUV optic's surfaces, EUV-induced plasma invokes various physical and chemical processes that might be crucial for the EUV optic's lifetime. For that reason it is important to understand the behavior of EUV-induced plasmas in detail. This requires a study of the spatio-temporal evolution of EUV-induced plasma and a development of suitable diagnostic methods.

There is an extensive range of papers covering the studies of pulsed radiation-generated plasmas. The first experimental work on EUV- 
induced plasma was the PhD thesis of Van der Velden [4], which followed his earlier publication on Particle-In-Cell with Monte Carlo collisions (PIC MC) simulation of such plasmas [7]. The PIC MC simulations were discussed in the $\mathrm{PhD}$ thesis as well and gave an insight into interaction of EUV-induced plasma with the optical surface. In the experimental part, an attempt to characterize an argon EUV-induced plasma with Langmuir probes was described. However, the author claimed those to be infeasible for studying EUVinduced plasma in a reliable manner.

A spectral study of a neon EUV-induced plasma in the focal spot of an EUV source, as well as under the irradiation by free-electron laser radiation, was conducted by Bartnik et al. $[8,9]$. Although they dealt

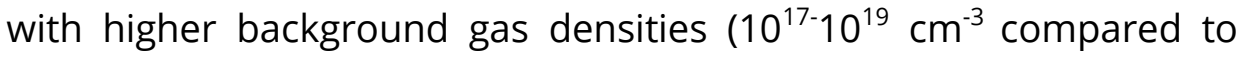
$10^{15}-10^{16} \mathrm{~cm}^{-3}$ in the lithography machine), they pointed out the ionizing role of hot secondary electrons emitted from neon atoms, which increased the plasma density in addition to direct photoionization. Recently, van der Horst et al. published a number of papers reporting the successful application of microwave cavity resonance spectroscopy (MCRS) for measuring $n_{\mathrm{e}}$ dynamics in an EUV-induced plasma $[5,10,11]$. One important result of their study was showing that the peak plasma density is proportional to the square of background gas pressure, i.e. $n_{\mathrm{e}}{ }^{\max } \sim p^{2}$. They explained this quadratic dependence by ionization due to secondary electrons, resulting from photoionization and, thus, emphasized the role of these secondary electrons in the formation of EUV-induced plasmas. The MCRS technique, despite its simplicity and non-invasive character, only deals with space-averaged values of $n_{\mathrm{e}}$ and is limited to a specific experimental geometry (microwave resonator cavity).

An experimental study of EUV-induced plasmas in a more spatiallyresolved and less geometry-specific way is still a challenge, since 
most methods of plasma diagnostics stumble upon short timescales and low signal levels (see [4]). However, due to modern advances in numerical simulations, one might build a rather strict model of the object and then use a wide set of experimental data to adjust the model input to match this data. This approach would make such a model an accurate instrument that can be used as an independent (and informative) study method. In this chapter, we present a study of the evolution of EUV-induced plasma in hydrogen, using the aforementioned approach. As an experimental diagnostic, we employ a small auxiliary electrode which we insert into plasma and then record the dynamics of current voltage characteristics for that electrode. This is similar to the probe technique introduced by Langmuir, [12] which has since been used for measuring plasma potentials, electron densities, and temperatures. Unfortunately, it is well known that, in some cases, such a probe can be a source of perturbation and the introduction there of can cause significant disturbance to the plasma. In the case of a low-density transient plasma, localized in a small volume (a few $\mathrm{cm}^{3}$ ), such as a typical EUV-induced plasma, the perturbation, even from a very small probe (a few tens of microns in dimensions), is comparable in scale with the whole plasma bulk size. This means, that the probe alters the plasma considerably, and the probe readings from the perturbed plasma might differ dramatically from the actual values from the plasma without a probe.

The disadvantages of the probe technique arising from the plasma perturbation can, however, be turned into an advantage when one resorts to accurate numerical modeling. In this chapter, we do it in the following way. For a range of probe potentials we simulate the evolution of EUV-induced plasma with the probe, using a twodimensional Particle-In-Cell Monte Carlo (2D PIC MC) model. The character of perturbations due to the probe varies with the probe 
potential, and, in our case, so does the evolution of the perturbed plasma. We validate our model against a wide range of probe potentials and obtain good agreement between the experimental probe curves and those calculated in simulations. Such thorough validation ensures that the model handles even such complicated cases as the perturbed plasma correctly and allows us to use the model as an independent method of studying the plasma. We then use our validated model to simulate the EUV-induced plasma in the same chamber without the probe. From these simulations, we deduce the dynamics of the plasma formation and draw conclusions on what processes take place therein. This chapter is organized in the following structure: section 2 describes the experimental setup. Subsections 3.1 and 3.2 of Results and discussion are dedicated to the experimental results that constitute the base for the further modeling. Finally, subsection 3.3 discusses the numerical modeling.

Subsection 3.3 is divided into three parts. The 1st part deals with validating the model against the results of the probe measurements: the essential step of making the model an accurate instrument. In the second part, the simulation of EUV-induced plasma formation with the validated model is discussed; we consider the spatiotemporal profiles of the plasma density and deduce the mechanisms responsible for the plasma formation. The third part of the numerical modeling subsection contains the discussion of the perturbations that the probe introduces into plasma and why our modeling approach is more fruitful than the direct extraction of any figures from the probe curves.

\subsection{Experimental setup}

All the experiments were conducted at the PROTO-2 experimental setup that is described in detail in [6]. It is a pulsed EUV source, 
based on a Z-pinch discharge in tin vapor operating at a repetition rate of $1.6 \mathrm{kHz}$. The pinch spectrum in the $10-20 \mathrm{~nm}$ range has a wellknown profile, such as described in [3]. The light from the pinch is collected by a grazing incidence collector and reflected into the clean chamber. This chamber is sealed tightly from the discharge chamber so it can be pressurized with a background gas to a pressure up to about $100 \mathrm{~Pa}$ while the pressure in the discharge chamber will be no higher than $5 \cdot 10^{-2} \mathrm{~Pa}$.

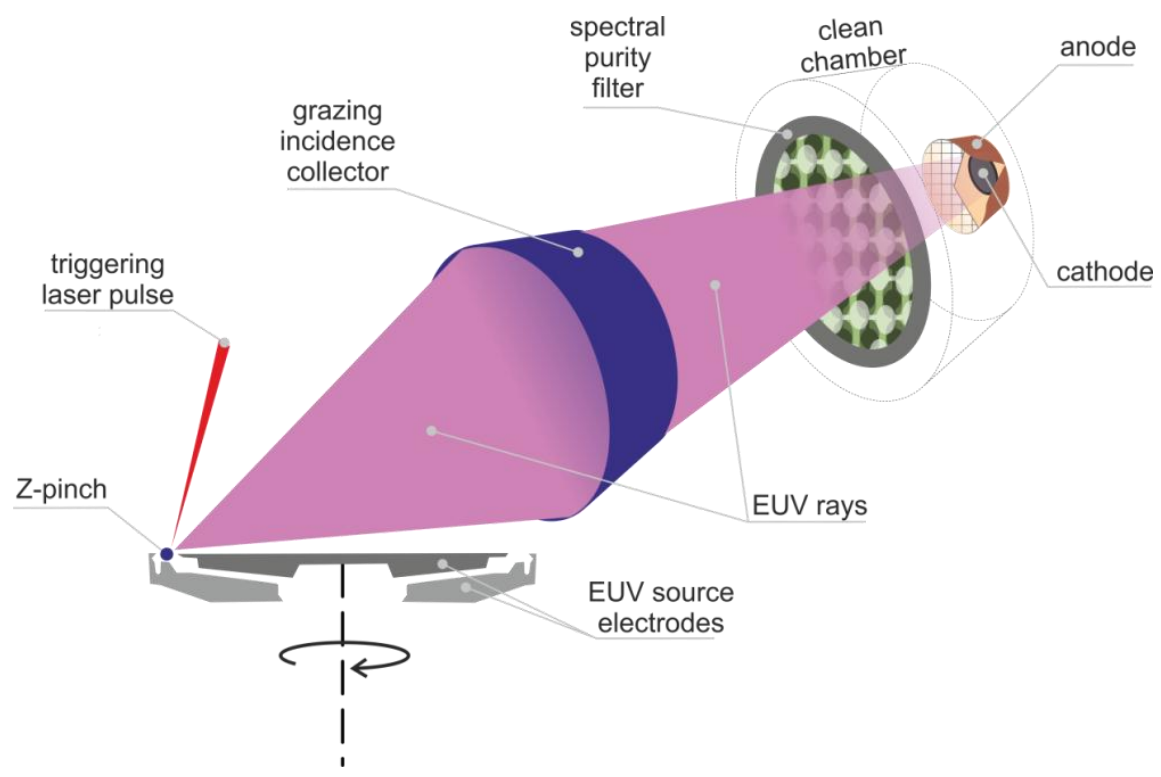

Figure 1: Schematic view of Proto-2 setup.

The EUV light enters the clean chamber through a spectral purity filter (SPF) window, which is a $200 \mathrm{~nm}$ thick multilayer Zr/Si foil [13]. This filter suppresses the IR, visible, and optical UV light from the pinch so it is mainly EUV and partly VUV light that enters the clean chamber. In the clean chamber, the setup for studying EUV-induced plasma in the vicinity of optical surfaces is assembled (See Figure 2). 
The main part of it is the sample holder. It is a round copper disc, $25.4 \mathrm{~mm}$ (1 inch) in diameter, placed at the focus of the EUV collector.

Note that the results described in subsection 3.1 were obtained with the probe removed from the chamber. A bias voltage, $V_{0}$, in the range of 0 to $-200 \mathrm{~V}$ can be applied to this disc with an external voltage supply so that it serves as a photocathode. The EUV light gathered by the collector is concentrated into a spot roughly $5-6 \mathrm{~mm}$ in diameter in the plane of the holder. The sample holder is equipped with a mount for 1 inch wafers, which allows placing various samples of surfaces. For instance, one can place a Mo/Si multilayer mirror to mimic the optical system of an actual EUV lithography machine. Throughout this chapter, however, only the measurements on a bare copper disc are discussed. Between the SPF and the sample holder, a grounded copper hollow cylindrical anode is placed. The anode has a diameter of $32 \mathrm{~mm}$ and a height of $40 \mathrm{~mm}$. On the filter side, the opening of the cylinder is covered with a nickel grid with a period of $1.5 \mathrm{~mm}$, which prevents the secondary plasma, created by secondary electron emission from the SPF, from entering the anode space. This was done so that the SPF and the $20 \mathrm{~mm}$ gap between the SPF and the grid could be omitted from the computational area in the numerical simulations. The axial symmetry and cylindrical shape are essential for modeling purposes since the model assumes cylindrical symmetry.

\subsection{Results and discussion}

Throughout our experiments we used hydrogen as a background gas. During each measurement, the gas pressure $\mathrm{P}_{\mathrm{H} 2}$ and the sample bias voltage, $\mathrm{V}_{0}$, were maintained at fixed values. 


\subsubsection{Photocurrent}

An important first stage of the experiment is measuring the current of photoelectrons from the sample due to the EUV irradiation. This is done in order to determine the starting value of the secondary electron yield, $Y_{\text {se, }}$ that is used as an input parameter in the model. The photocurrent was measured at a 220 Ohm resistor, connected between the sample holder and the anode through the DC blocking capacitor (see the Figure 2).

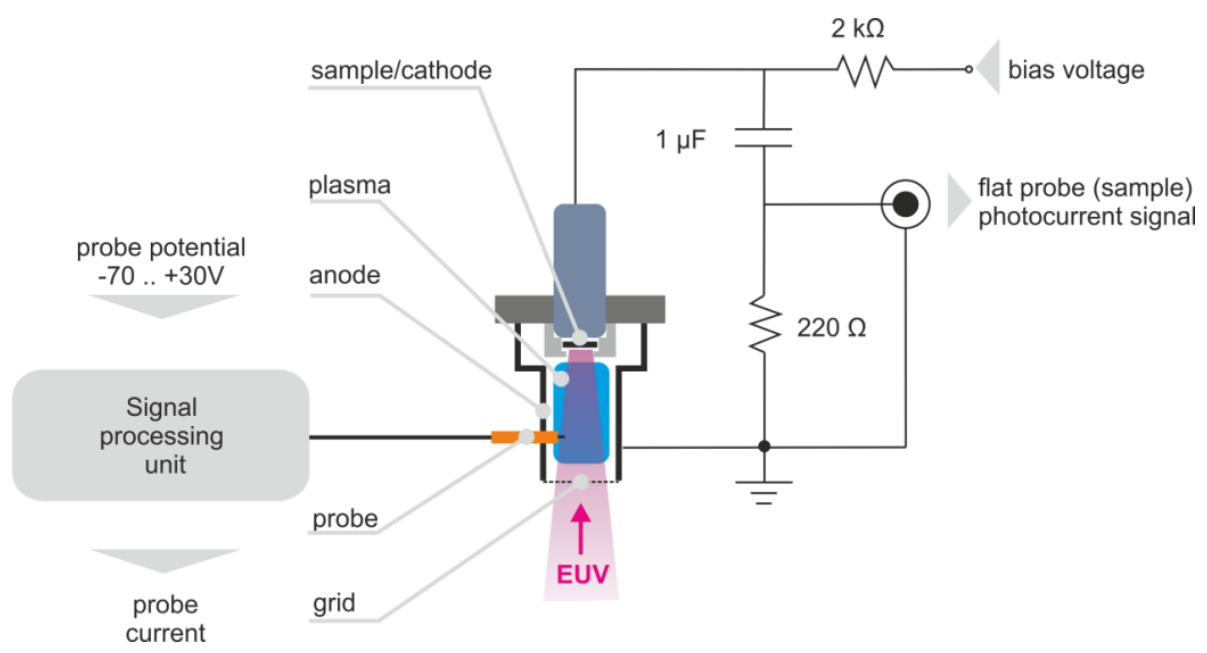

Figure 2: Schematic view of the clean chamber EUV-induced plasma diagnostic setup.

Because of space charge effects, a fraction of the secondary electrons are repelled back towards the sample, which leads to the underestimation of the photocurrent magnitude. To lessen the space charge effect, a negative voltage, $V_{0}$, is applied to the sample. The resulting pulse for $V_{0}=-190 \mathrm{~V}$ is shown in the figure 3a. The secondary electron current has a peaked shape with the maximum value at $t=80 \mathrm{~ns}$ and the width $\tau_{\mathrm{EUV}}=100 \mathrm{~ns}$ at half-maximum. 
By integrating the pulse, we obtain the charge, $\mathrm{Q}_{\mathrm{se}}$, emitted from the sample. The dependence of $\mathrm{Q}_{\text {se }}$, recorded as a function of $\mathrm{V}_{0}$, is shown in the figure $3 \mathrm{~b}$. It can be seen that the charge gradually increases with bias voltage, with the maximum, $\mathrm{Q}_{\mathrm{se}}{ }^{\max }=0.6 \mathrm{nC}$, obtained for the most negative voltage we could apply, which was $190 \mathrm{~V}$.

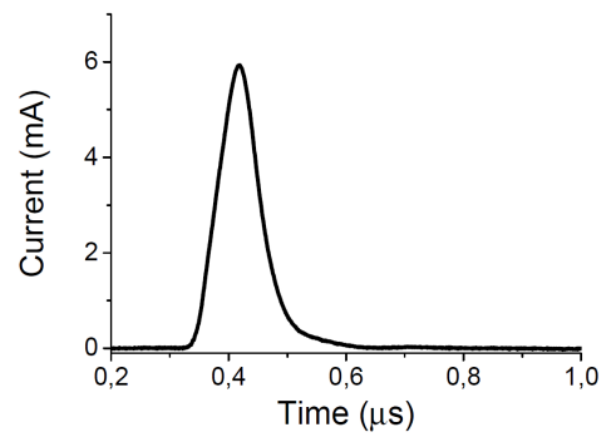

a)

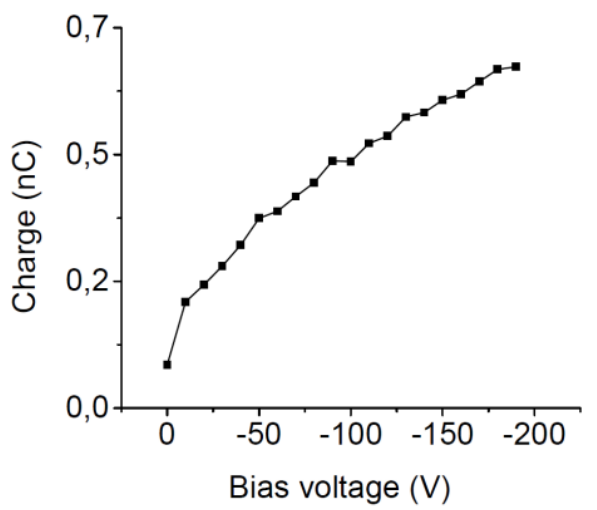

b)

Figure 3: a) The shape of the EUV pulse in vacuum at the sample with $\left.V_{0}=-100 \mathrm{~V} ; b\right)$ The pulse integral against $V_{0}$.

We use this value to determine the secondary electron yield:

$$
Y_{s e}=\frac{Q_{s e}^{\max }}{e} \cdot \frac{h v_{E U V}}{E_{E U V}}
$$

with $E_{E U V}$ the EUV dose per pulse (which we measured to be about $5 \mu \mathrm{J})$ and $h v_{\mathrm{EUV}}=92 \mathrm{eV}$, the EUV photon energy. This gives $\mathrm{Y}_{\mathrm{se}}=0.008$, which is a typical value for copper under EUV light [14]. Since the secondary electron yield varies significantly (orders of magnitude) with surface material and anything adsorbed or deposited on it, knowing $\mathrm{Y}_{\mathrm{se}}$ is important for computational purposes. 


\subsubsection{Probe measurements}

For the probe measurements, we insert the auxiliary electrode perpendicular to the assembly axis at a $28 \mathrm{~mm}$ distance from the sample holder. The probe tip was made of steel and had a diameter of $0.5 \mathrm{~mm}$ and a length of $5 \mathrm{~mm}$. The probe tip was centered so that its middle is centered on the assembly axis (see Figure 2). The probe was connected to a signal processing unit capable of measuring currents with $\mathrm{RC} \approx 100$ ns and a current noise level $i_{n} \approx 10 \mathrm{nA}$ (RMS). In order to obtain the current-voltage characteristics, a series of probe current pulses was recorded at different probe potentials. This series was then sliced at different time moments in order to obtain the IV curves. Examples of curve dynamics for $\mathrm{P}_{\mathrm{H} 2}=20 \mathrm{~Pa}$ and $\mathrm{V}_{0}=-50 \mathrm{~V}$ are shown in Figure 4.

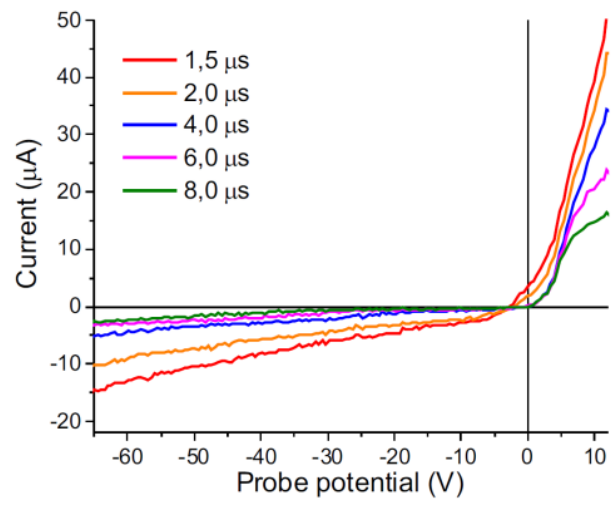

a)

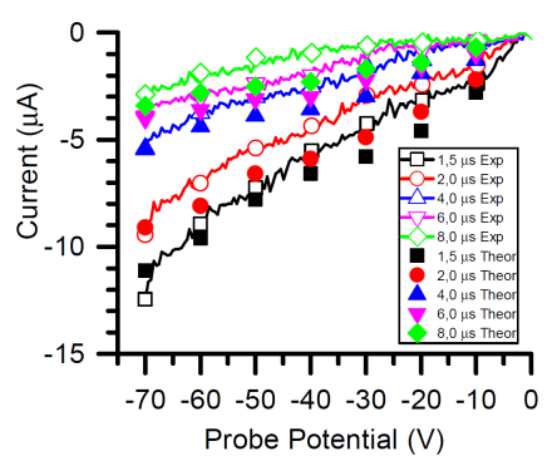

b)

Figure 4: a) The probe current-voltage curves for $20 \mathrm{~Pa} \mathrm{H}_{2}$ and $-50 \mathrm{~V}$ bias voltage, b) The comparison between the experimental results and the numerical calculations.

As can be seen, these curves resemble classical probe characteristics with the ion part at $\mathrm{V}<0$ and the electron part between $\mathrm{V}=0$ and $\mathrm{V}=$ $V_{p}$, the plasma potential. Having obtained the dynamics of the probe 
curves, we can use them for validating our model. The process of model validation is described in the following subsections.

\subsection{Numerical modeling}

\subsubsection{Validating the model input}

The idea of validating the numerical model against the probe curve dynamics arises from the following circumstances. If the size of the sheath is the same order as the plasma bulk dimensions, the perturbation becomes a significant factor in the plasma behavior. This might hinder the direct extraction of the plasma parameters from the probe curves. However, such a perturbation might come as an advantage when it comes to numerical modeling. As the probe perturbation creates an intricate distribution in particle density and electrostatic potential, handling such a configuration would be a challenge to the numerical simulations. Because of that, making sure that the numerical experiment handles the perturbation correctly and gives accurate values of probe current for a wide range of probe potentials is a good task for model validation.

Every value of the probe potential in this case serves as a separate case for determining the plasma dynamics. The model used for simulation is a two-dimensional Particle-in-Cell electrostatic plasma model with a Monte-Carlo collisions scheme, described in detail in [15]. The model includes the differential cross-sections for both elastic and inelastic scattering of electrons off $\mathrm{H}_{2}$ molecules. The model also includes the kinetic description, not only for electrons, but for all relevant ions species, which is essential for the description of the plasma sheath dynamics. This model was used for simulations in [16] and proved to yield good agreement with the experimental 
results. Hence, we use the model for the self-consistent simulation of the plasma with the probe included as an electrode in the simulation domain.

Since the model code is two-dimensional $(R, z)$, we couldn't simulate a horizontal probe, so, instead, we simulated it as a vertical probe with the same dimensions $\left(2 R_{p}=0,5 \mathrm{~mm}, L_{p}=5 \mathrm{~mm}\right)$, with the middle of the probe tip at the same position $(z=28 \mathrm{~mm})$ as in the experiment (see section 2).

This can be done if the sheath geometry is close to spherical and its dimensions are larger than or of the order of the probe size. This can take place in the case of a negatively biased probe in a low-density plasma and holds for our case, as we will show later in the chapter. However, if the probe potential is positive, one can still expect a thin cylindrically-shaped sheath, in which case a horizontal probe would differ significantly from a vertical one. Therefore, we limited our simulations to the range of probe potentials lower than zero.

In order to obtain the current-voltage characteristics, a separate calculation was run for each of the values of the probe potential between -70 and $-10 \mathrm{~V}$ with a $10 \mathrm{~V}$ step. The obtained I-V curves are shown in Figure 4b. As can be seen, the simulated dynamics of the probe curves are similar to those of the experiments. There is also good qualitative agreement between the simulation results and the experimental curves at short times, $t<4 \mu \mathrm{s}$.

However, at longer timescales, a difference of about 1- $2 \mu \mathrm{A}$ between the simulation and the experiment results in a significant error in the current. The reason for this discrepancy is the difference between the radial probe geometry used in the experiment and the axial probe used in the simulation. Beside the orientation of the probe 
itself, the potential distributions in the sheaths are also differ in their orientation. The potential gradients around the probe, thus, add to the deviation from the axial symmetry. As the sheath around the probe expands, along with the decay of the plasma, the gradients decrease.

This makes the difference between the probe orientations more significant and, therefore, contributes to the error in the current. Therefore, in this case, we paid more attention to the agreement at early times, $t \leq 4 \mu \mathrm{s}$, which is less affected by the non-axially symmetric experimental geometry.

An electrode in the middle of the chamber, such as our probe, makes the system rather complicated, with intricate potential distributions, and density gradients, as well as the transient processes at microsecond timescales. Having successfully simulated such complicated system with our model, we can rely on it as an independent accurate instrument that provides us with the actual parameters of our plasma. For that we turn back to the simulations in the clean chamber without the probe.

\subsubsection{EUV-induced plasma formation under different conditions}

In this section we present a series of simulations of EUV-induced plasma formation during the EUV pulse for different pressures and bias voltages. In Figure $5 a$, the electron density fields and potential distributions for the $\mathrm{P}_{\mathrm{H} 2}=20 \mathrm{~Pa}$ and $\mathrm{P}_{\mathrm{H} 2}=100 \mathrm{~Pa}$ at $\mathrm{V}_{0}=-190 \mathrm{~V}$ are shown. Both cases exhibit a region of high electron density $\left(n_{e}>3 \cdot 10^{9} \mathrm{~cm}^{-3}\right)$ propagating from the cathode at the bottom of the chamber. However, the propagation characters differ for the two pressures. It should be noted that, in both cases, a plasma 
sheath near the cathode is formed at the times as early as $40 \mathrm{~ns}$, so the potential drop of $190 \mathrm{~V}$ occurs over a small distance (a few $\mathrm{mm}$ ) from the biased surface.

Therefore, the secondary electrons are accelerated to high energy over a short path without any collisions. The main loss mechanism for electrons at this energy is electron impact ionization. The ionization cross-section for this energy is $\sigma_{i z}(190 \mathrm{eV})=0.7 \cdot 10^{-16} \mathrm{~cm}^{2}$ [17]. The corresponding mean free paths are shown in Table 1.

Table 1. Pressure, gas concentration and corresponding mean free paths into the chamber.

\begin{tabular}{|c|c|c|}
\hline Pressure, $\mathrm{Pa}$ & $n_{a}, 10^{15} \mathrm{~cm}^{-3}$ & $\lambda_{\mathrm{iz}} \mathrm{cm}$ \\
\hline 20 & 4.9 & 2.5 \\
\hline 100 & 24 & 0.5 \\
\hline
\end{tabular}

As can be seen from Table 1, the mean free path for impact ionization at $20 \mathrm{~Pa}$ is the order of the chamber size, which is $32 \mathrm{~mm}$ in diameter. Therefore, the accelerated electrons move through the chamber in a collisionless-like way. Because of that, the region of high electron density grows mostly in the upward direction. Conversely, the mean free path for $100 \mathrm{~Pa}$ is less than the chamber size, so the secondary electrons experience multiple collisions with neutral atoms and molecules, which results in the "blurring" of the upward-directed electron momentum, and the plasma column expands noticeably in the radial direction in the bottom part of the chamber. 


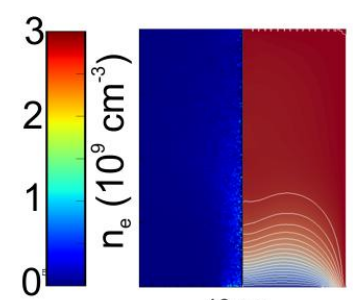

40 ns

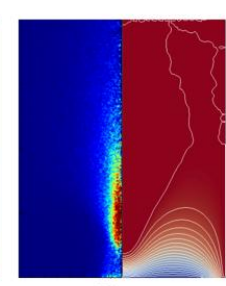

$60 \mathrm{~ns}$

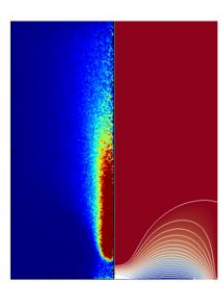

80 ns

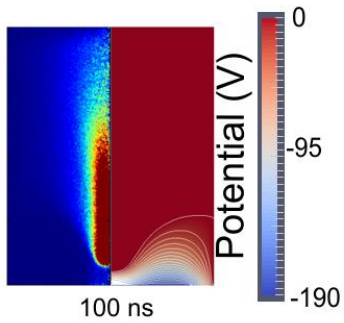

a)

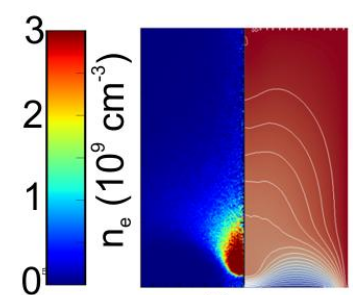

$40 \mathrm{~ns}$

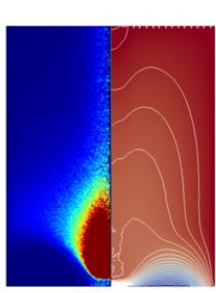

$60 \mathrm{~ns}$

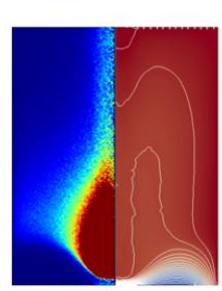

80 ns

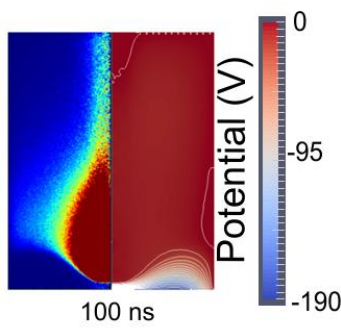

100 ns

b)

Figure 5: Simulated electron densities at $-190 \mathrm{~V}$ bias voltage in $20 \mathrm{~Pa}$ (a)and 100 $\mathrm{Pa}$ (b) The field dimensions in all simulation results are $2 \mathrm{R} \cdot \mathrm{h}=32 \cdot 40 \mathrm{~mm}^{2}$.

The situation differs for the case of weak fields. Figure 6 depicts the plasma formation for $\mathrm{V}_{0}=-50 \mathrm{~V}$. In these cases, before the sheath is formed, a comparatively small potential drop occurs at long paths. This makes electrons lose their energy over this path in elastic collisions. In the case of $\mathrm{P}_{\mathrm{H} 2}=20 \mathrm{~Pa}$, however, a sheath is formed during the EUV pulse, which allows a small number of electrons to gain the energy sufficient for ionization over the thin sheath. Because of this, a small region of impact ionization is formed at the bottom of the chamber at $t>80 \mathrm{~ns}$.

Contrary to the low pressure case, at $\mathrm{P}_{\mathrm{H} 2}=100 \mathrm{~Pa}$, the sheath is only formed at $t>160 \mathrm{~ns}$, when secondary electron emission is low, and the accelerated secondary electron contribution is insignificant 
compared to photoionization. On the other hand, at such pressure, photoionization plays a considerable role in the plasma formation, as can be seen from the uniform plasma column forming along the chamber axis during the whole EUV pulse.

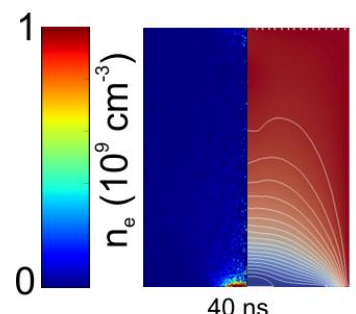

40 ns

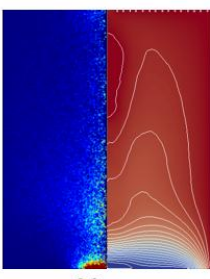

$80 \mathrm{~ns}$

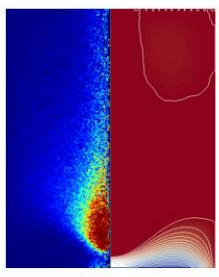

$120 \mathrm{~ns}$

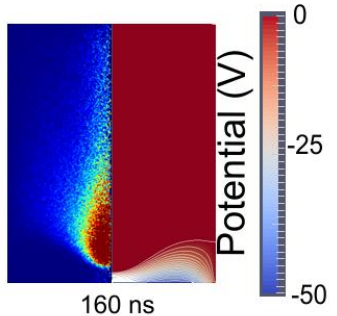

$160 \mathrm{~ns}$

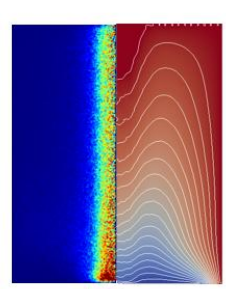

$120 \mathrm{~ns}$

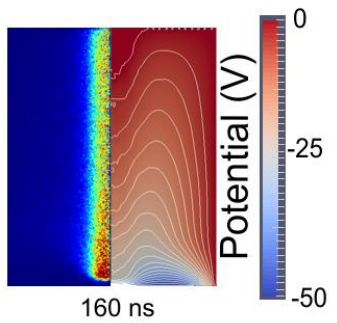

b)

Figure 6: Simulated electron densities at -50 Volt bias voltage in $20 \mathrm{~Pa}$ (a) and100 $\mathrm{Pa}$ (b)

The probe influence on the plasma can be easily seen in comparison with the plasma behavior without a probe, as in Figure 7. As can be seen, a probe creates a large sheath, which expands with time as the plasma decays. Here we should make two remarks about the sheath configuration:

1. The sheath has a spherical shape, which justifies our using the vertical probe geometry instead of horizontal in the 
simulations. However, the radial effects in the potential distribution might become an issue at long timescales. This has been discussed in Subsection 3.3.1.

2. The sheath dimensions are of the order of the plasma bulk. These are also comparable with the scales of the density gradients.

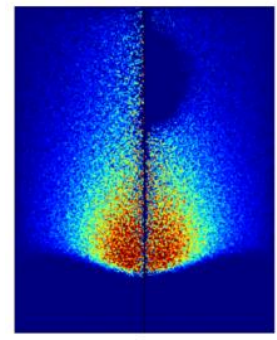

$\mathrm{t}=1 \mu \mathrm{s}$

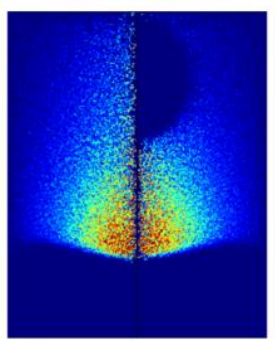

$t=2 \mu s$

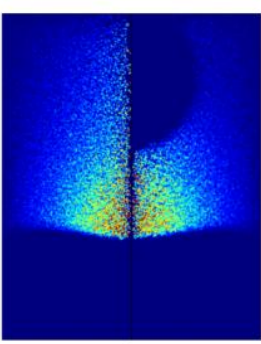

$\mathrm{t}=4 \mu \mathrm{s}$

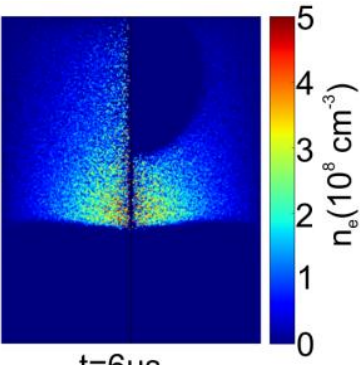

$t=6 \mu \mathrm{s}$

Figure 7: Simulated electron density fields for the EUV-induced plasma in the empty chamber (left halves) and in the presence of a probe (right halves) biased to $-30 \mathrm{~V}$.

Because the probe current-voltage curve is actually the currentvoltage characteristic of the sheath, all quantities extracted from the current-voltage curves are, in fact, the spatial average values, averaged over the sheath volume. When the sheath dimensions are the order of the density gradients in the unperturbed plasma or worse, the whole plasma bulk dimensions, the probe readings turnout to be uninformative and of questionable relation to the actual plasma parameters. 
Using the probe curves for validating the strict numerical model, as we did in this chapter, does not have this disadvantage and yields an adequate spatio-temporal picture of the plasma evolution.

\subsection{Conclusion}

Throughout this chapter we described the combined experimental and computational method of studying EUV-induced plasmas: a transient low density plasma, which is a challenge for plasma diagnostics. The main diagnostic tool we used here was a 2D ParticleIn-Cell model with Monte Carlo collisions. However, we needed to verify the accuracy of that model as a diagnostic tool. For that, we conducted a series of experimental measurements to validate the model against the obtained data. Hence, our research consisted of two parts. In the experimental part we performed the time-resolved electrostatic probe measurements, thus obtaining a dynamic of current-voltage curves. The computational part was based on using these curves as the base for model validation. The goal there was to make sure that the model handled the computation of a complicated system, such as a plasma perturbed by a probe, with satisfactory agreement with experimental results. Having confidence in the model, we then ran a number of simulations of EUV-induced plasma in the chamber without the probe. This we did for a set of pressures and bias voltages so as to obtain the spatio-temporal picture of plasma formation during the EUV pulse.

The results of the simulations revealed a variety of regimes for the plasma formation in the vicinity of an electrically biased surface. It was demonstrated, that, beside photoionization by EUV photons, the secondary electrons can also contribute to the plasma formation when the electric field is present in the chamber. However, the role 
of the impact ionization depends strongly on the plasma conditions, i.e. gas pressure and the bias voltage at the irradiated surface. The variation of the plasma behavior in relation to the parameters that can be controlled manually gives a possibility to control the plasma in a predictable way, which can be useful in managing the EUVinduced plasma interaction with optical surfaces.

Another thing that should also be pointed out is the universal character of the approach that we employed in this work. The availability of large amounts of computing power, in conjunction with a large accumulated set of cross-sections and level structures for a variety of atoms and molecules, gives the possibility to build strict models and then validate these based on the data obtained from simple experiments. This approach is beneficial in two ways. First, it allows the range of the experimental techniques to be extended beyond the direct interpretation of the experimental results. Second, it can provide the researcher with valuable knowledge of plasma parameters for which no reliable diagnostic method is developed.

Our compound experimental and computational method, thus, allows one to view both the experimental and computational works in a different and novel way. 


\section{References}

1. Attwood D 2007 Soft x-rays and extreme ultraviolet radiation: principles and applications. (Cambridge university press.)

2. Louis E, Yakshin A E, Tsarfati T, Bijkerk F 2011 Progress in Surf. Science $86255-294$.

3. Banine V Y, Koshelev K N, Swinkels G H P M 2011 J. Phys. D: Appl. Phys. 44253001.

4. van der Velden H L 2008 Radiation generated plasmas Doctoral Thesis Eindhoven University of Technology Netherlands.

5. van der Horst R M et al. 2014 J. Phys. D: Appl. Phys. 47302001.

6. A Dolgov et al 2015 Plasma Sources Sci. Technol. 24035003.

7. van der Velden M H L, Brok W J M, van der Mullen J J A M, Goedheer W J, and Banine V 2006 Phys. Rev. E 73.3036406.

8. Bartnik A et al. 2013 Laser and Particle Beams 31 195-201.

9. Bartnik A, Fiedorowicz H and Wachulak P 2014 Physics of Plasmas (1994-present) 21073303.

10. van der Horst R M, Beckers J, Osorio E A and Banine V Y 2015 J. Phys. D: Appl. Phys. 48285203.

11. van der Horst R M et al. 2015 J. Phys. D: Appl. Phys. 48432001.

12. Mott-Smith H M and Irving Langmuir 1926 Physical Review 28 727763.

13. Bibishkin M S, Chkhalo N I, Gusev S A, et al. 2008 Proceedings of SPIE 7025702502.

14. Day R H et al. 1981 Journal of Applied Physics 52.11 6965-6973.

15. Astakhov D I et al. 2015 Plasma Sources Sci Technol. 24055018.

16. Astakhov D I et al. 2016 J. Phys. D: Appl. Phys. 49295204.

17. Astakhov D 2016 Numerical study of extreme-ultra-violet generated plasmas in hydrogen Doctoral Thesis University of Twente Netherlands. 


\title{
4. Comparison of $\mathrm{H}_{2}$ and $\mathrm{He}$ carbon cleaning mechanisms in extreme ultraviolet induced and surface wave discharge plasmas
}

\begin{abstract}
.
Cleaning of contamination of optical surfaces by amorphous carbon $(a-C)$ is highly relevant for extreme ultraviolet (EUV) lithography. We have studied the mechanisms for a-C removal from a Si surface. By comparing a-C removal in a surface wave discharge (SWD) plasma and an EUV-induced plasma, the cleaning mechanisms for hydrogen and helium gas environments were determined. The C-atom removal per incident ion was estimated for different sample bias voltages and ion fluxes. It was found that $\mathrm{H}_{2}$ plasmas generally had higher cleaning rates than He plasmas: up to seven times higher for more negatively biased samples in EUV induced plasma. Moreover, for $\mathrm{H}_{2}$, EUV induced plasma was found to be 2-3 times more efficient at removing carbon than the SWD plasma. It was observed carbon removal during exposure to $\mathrm{He}$ is due to physical sputtering by $\mathrm{He}^{+}$ ions. In $\mathrm{H}_{2}$, on the other hand, the increase in carbon removal rates is due to chemical sputtering. This is a new $C$ cleaning mechanism for EUV-induced plasma, which we call "EUV-reactive ion sputtering".
\end{abstract}

\section{Introduction}

According to the International Technology Roadmap for Semiconductors (ITRS), extreme ultraviolet lithography (EUVL) is currently the most advanced technology for the fabrication of integrated circuits with characteristic half-pitch, hp $\leq 22 \mathrm{~nm}$ [1]. Multilayer mirrors (MLM) are the basic optical element in EUV 
lithography. At an operating wavelength of $13.5 \mathrm{~nm}$, MLMs consist of approximately 50-60 bi-layers of Mo:Si that are $6.7 \mathrm{~nm}$ thick. The mirrors are often covered with a protective layer that is $1.5-2 \mathrm{~nm}$ thick. To obtain the desired optical resolution, the mirrors must have a surface roughness much less than the wavelength, with reported values being approximately 0.2-0.3 nm. Thus, multilayer mirrors in EUV lithography are expensive, high-technology items, making it desirable to extend their useful lifetime as much as possible.

Previous research has demonstrated that MLMs lose their reflectivity due to the pollution of their surfaces with amorphous carbon, and/or surface oxidation, induced by intense EUV radiation [2-5]. In order to ensure optimal image resolution and uniform exposure, the EUVL optical system consists of 6-10 multilayer mirrors. In these circumstances, even a small loss of reflectivity for each mirror simultaneously ( 1-2\%) leads to a significant deterioration in optical throughput. For example [6,7], a carbon film just a few nm thick would already cause such loss of MLM reflectivity. In UHV conditions, where the rate of accumulation of amorphous carbon is limited by the partial pressure of hydrocarbons, the growth rate of the carbon layer can be quite high, ranging from $0.001 \mathrm{~nm} / \mathrm{hr}$ to $0.01 \mathrm{~nm} / \mathrm{hr}$, depending on the precise EUV illumination and partial pressures $[8,9]$.

Given that EUVL requires a long MLM lifetime (i.e. 30000 hr) [10,11], it is obvious that cleaning mechanisms for MLMs are required. Moreover, such cleaning systems should be realized in such a way that interruptions to operation are minimized, i.e. in situ, without affecting the rest of the equipment or the lithographic process. In terms of quantities, such in situ cleaning should remove carbon with sufficient efficiency (at least faster than it deposits) and, at the same 
time, the capping layer of the mirror should not suffer damage over the entire period of the MLM's life.

At present, atomic hydrogen is used to clean MLMs [12-18], because the majority of basic hydrogen compounds are volatile. However, the efficiency of atomic hydrogen cleaning of amorphous carbon is extremely low [19] particularly in comparison with the surface recombination probabilities [20]. Moreover, atomic hydrogen generates heat (e.g., radiative heating from a hot filament), creating a high radiation and thermal load on the MLM surface that affects the speed and selectivity of cleaning. The efficiency of removing amorphous carbon from the MLM surface using plasmas is much higher than atomic hydrogen cleaning [19]. A low-temperature lowpressure plasma, even at low densities, provides fast, efficient and accurate carbon cleaning. This is why the generation and management of such plasmas, near the MLM surface during EUVL operation, is of special importance for the development of continuous in situ mirror cleaning technology. The EUV-photon energy of $91.8 \mathrm{eV}$ is sufficient to generate a cascade of photons and secondary electrons. The EUV-induced secondary electrons collide with the background gas (mostly molecular hydrogen) to form a plasma above the surface of the EUV optical element. Furthermore, intensive EUV radiation, along with the flux of charged particles from the plasma, can induce processes on the surface with the participation of gas molecules.

This chapter investigates the mechanism of amorphous carbon cleaning in EUV-induced plasma. To exclude surface sputtering and separate the influence of physical and chemical processes, the background gases were limited to molecular hydrogen and helium. Moreover, to determine the role of photons, analogous experiments were conducted in a low-temperature discharge plasma with similar 
ion energies and plasma densities. By comparing cleaning rates between these different regimes, the roles of ion energy, EUV radiation, and chemical activity were clarified.

\subsection{Samples and diagnostics of amorphous carbon}

Previous research has shown that magnetron sputtered carbon films are structurally and chemically similar to carbon films that grow during EUV illumination [21]. Hence, in this work, magnetron deposited amorphous carbon films are used as model contamination layers. Films of carbon were deposited on silicon wafers and model MLMs (40 bi-layers of Mo/Si, each $\sim 6.9 \mathrm{~nm}$ thick). The as-deposited carbon layers had thicknesses of $\sim 20$ and $\sim 10 \mathrm{~nm}$ for the silicon wafer and MLM samples, respectively. Amorphous carbon layer thicknesses were measured using Raman spectroscopy (RS) and the Energy Dispersive Spectroscopy (EDS), i.e. X-ray fluorescence analysis (XRF).

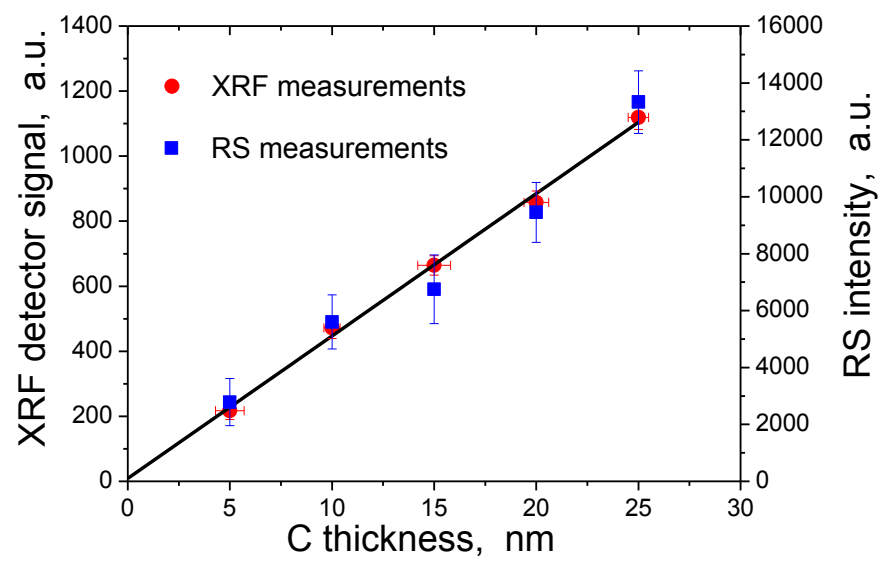

Figure 1. The magnetron-deposited carbon peak intensities for XRF (EDS) and RS versus the carbon thickness, measured by ellipsometry and quartz mass balance the deposition). 
Figure 1 shows a typical calibration curve for RS and XRF. The symbols show intensity of the EDS and RS signals (carbon peak intensity for the EDS method and integral of the D- and G-peak region in RS) versus carbon film thickness. The deposited carbon mass was measured during deposition by a quartz mass balance. The layer thickness was measured using spectroscopic ellipsometry after deposition. A linear regression of the data results in a measurement accuracy of $\pm 0.5 \mathrm{~nm}$ (whatever a monolayer is) for XRF and $\pm 2 \mathrm{~nm}$ for RS. Since XRF provides higher precision, it was used for the data presented.

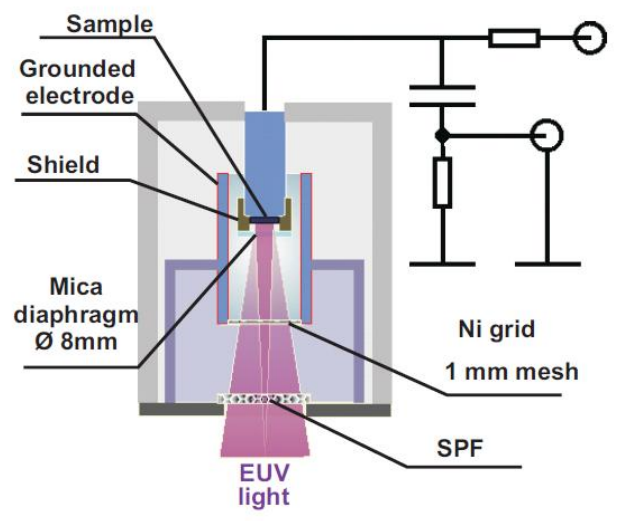

Figure 2. Set up of the experiment for carbon cleaning in EUV-induced plasma.

\subsection{EUV-induced plasma}

A schematic of the EUV-induced plasma interaction chamber and sample surface is presented in figure 2 . The interaction chamber is capable of operating at background pressures of $\sim(3-5) \cdot 10^{-8} \mathrm{Torr}$, which is maintained by separating the interaction chamber from the EUV source with a Zr/Si spectral purity filter ( 40\% transmission at 
$13.5 \mathrm{~nm})$. The interaction chamber can be supplied with either $\mathrm{H}_{2}$ or $\mathrm{He}$, which are passed though a cold-trap to remove contaminants before the gas is introduced to the chamber.

The plasma is initiated by a short impulse of EUV $13.5 \mathrm{~nm}$ radiation, incident on the sample surface. The EUV source is a Sn-based, discharge Z-pinch, which operation principle has been described in detail elsewhere [22]. The source emits 100 ns pulses of broadband EUV radiation at a repetition rate of $1.5 \mathrm{kHz}$. After focusing and filtering, the spectrum is centered on $13.5 \mathrm{~nm}$.

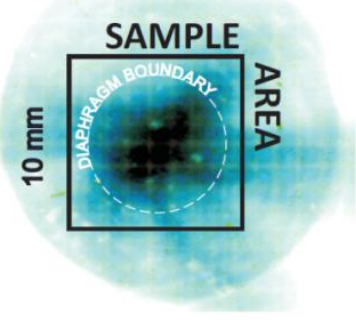

(a)

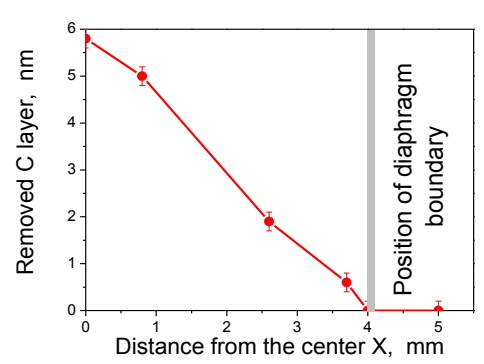

(b)

Figure 3. (a) EUV exposure pattern measured by sensitive foil and (b) C cleaning profile. To show (b) cleaning rate profile for obtaining the cleaning rate averaged on the profile to recalculate it then into the $C$ yield atoms/ion since the total number of ions (charge of ions) came to the sample surface (on $1 \mathrm{~cm}^{2}$ per a EUV pulse) is measured as averaged on the sample area (inside the mica diaphragm, the position and size of which is indicated in yellow). 
An image of the intensity profile of the EUV radiation at the focus is presented in figure 3a. The collector mirror's focal spot and a halo of scattered EUV radiation of 1-2 mm, due mainly to the roughness of the mirror surface are clearly visible. To eliminate most of the scattered EUV radiation, a diaphragm of synthetic mica $(\varnothing=8 \mathrm{~mm})$ was placed on top of the samples (see figure $3 a$ ). Figure $3 \mathrm{~b}$ presents a radial profile of the EUV radiation intensity inside the diagraph. It can be seen that the intensity of the EUV radiation reduces significantly towards the edge of the exposure zone, while the central area of $3 \mathrm{~mm}$ diameter has a relatively uniform distribution. The intensity profile of the EUV radiation incident on the sample was taken into account in the analysis presented below. The pulse energy was limited by the diaphragm to $0.042 \mathrm{~mJ}$, whereas the average power was estimated to be $0.13 \mathrm{~W} / \mathrm{cm}^{2}$.

As a consequence of the fixed EUV source geometry, a cylindrical biasing electrode system was used. The sample plays the role of the cathode, being held at negative biases, down to -200 V. A metal cylinder, $30 \mathrm{~mm}$ in diameter and $\sim 60 \mathrm{~mm}$ long, served as a grounded anode, with the sample centered on the axis of the cylinder, as shown in figure 2. In background-only tests, the photoelectron current has a duration that is nearly identical to that of the EUV pulse duration, while the peak current depends on the applied bias voltage (see figure $4 a$ ). In the presence of higher pressures (5-45 Pa), a similar photoelectron current pulse was observed. However, the tail

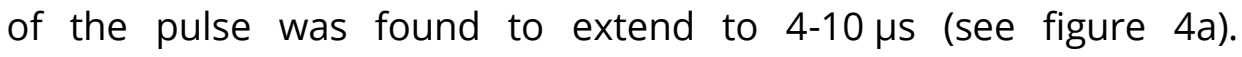
Moreover, the current in the tail was found to increase for more negative bias voltages. The tail is a product of ionization by accelerated 'hot' secondary photoelectrons, as well as photoionization of gas molecules and atoms. However, it should be noted that the first process is, apparently, the main one, based on the known $\mathrm{H}_{2}$ and He photoionization cross-sections, and the low 
pressures used in our experiments. The total charge developed by the EUV pulse (i.e. the number of electrons freed by ionization as well as photoelectrons) is estimated by integrating the current.

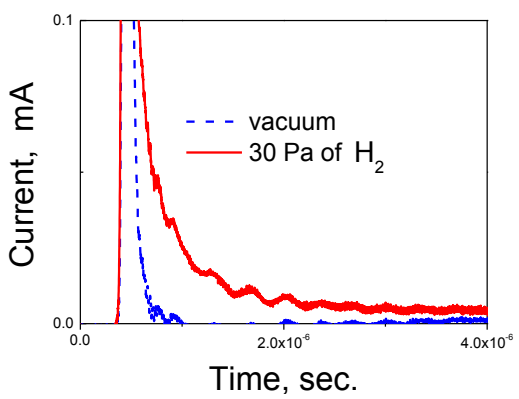

(a)

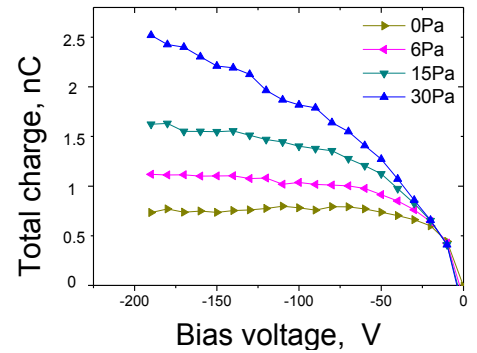

(b)

Figure 4. (a) Time evolution of sample current in vacuum and gas (used to characterize the plasma formation). The appearance a slow tail in the sample current is clearly visible for an EUV pulse in the presence of a gas. The slow tail corresponds to the plasma decay current. The decay time is determined by slow ion motion in the plasma over the sample surface, where ions are accelerated by the plasma sheath in front of the sample surface, before they are incident on the sample surface. (b) Integrated CV characteristics for EUV diode. The total charge (the integral of the current curve, see figure 6) incident on a sample covered by carbon film vs the applied bias voltage at the different $\mathrm{H}_{2}$ pressures.

Figure $4 \mathrm{~b}$ shows the total charge collected following the EUV pulse versus the applied bias voltage (volt-coulomb characteristics) in vacuum and at different values of $\mathrm{H}_{2}$ pressure. The difference between sample currents in a gas and vacuum corresponds to the charge generated in the gas, i.e. plasma charge, and is related to the total ion charge collected by the sample surface. Assuming that the plasma is quasi-neutral, the total ion charge was used to estimate 
the number of ions formed in the volume of the EUV-induced plasma above the sample. It was also used to estimate the per-pulse ion flux, incident on the surface, for each applied bias voltage.

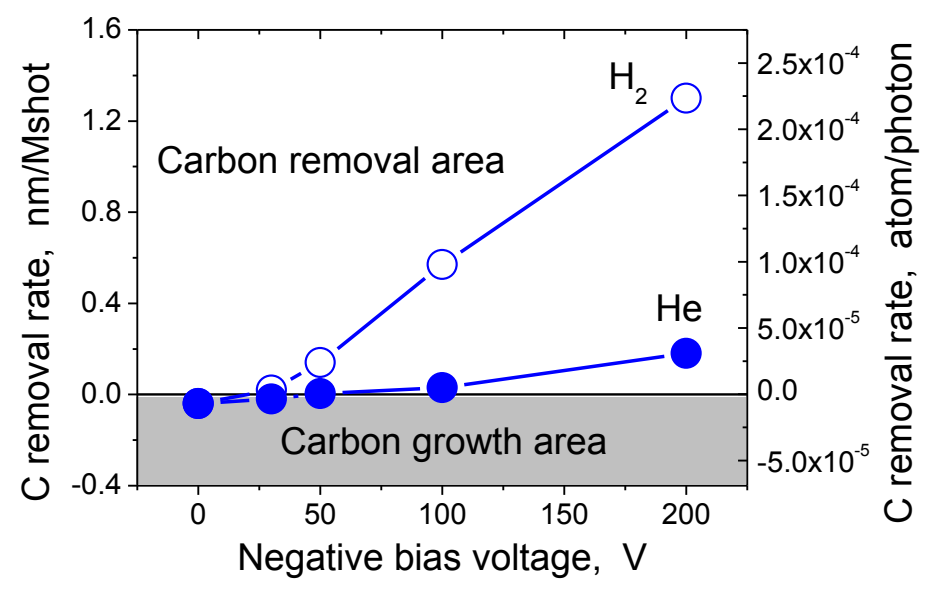

Figure 5. The averaged thickness of removed carbon in EUVinduced $\mathrm{H}_{2}$ and He plasma vs the applied negative bias. The chamber pressure was $3 \mathrm{~Pa}$, the EUV pulse energy was $85 \mu \mathrm{J}$ $\left(\sim 5.10^{12} \mathrm{ph} / \mathrm{cm}^{2}\right.$ pulse), and EUV dose was $10^{7}$ pulses. Negative values in the removed carbon thickness correspond to the growth of the carbon layer under the EUV exposure.

The radial profile of removed carbon approximately matches the profile of EUV radiation (see figure $3 b$ ). Figure 5 presents the rate of carbon removal at the center of the EUV spot in $3 \mathrm{~Pa} \mathrm{H}_{2}$ and $\mathrm{He}$ plasmas for various bias voltages. It can be seen that the rate of carbon removal increases with increasing bias voltage. Moreover, this rate is significantly higher for $\mathrm{H}_{2}$ plasmas, compared to that for He plasmas. The increase in bias voltage leads to an increase in 
current and the energy of ions incident on the sample surface, which, should, naturally, lead to an increase in the rate of carbon removal. Note that the differences between the volt-coulomb characteristics for $\mathrm{H}_{2}$ and $\mathrm{He}$ EUV-induced plasma are insignificant, i.e. the difference between ionic flux for $\mathrm{H}_{2}$ and $\mathrm{He}$ is insignificant. This means that the number and kinetic energy of the particles incident on the sample surface for the two different plasmas are the same. Similarly, $\mathrm{He}^{+}$and $\mathrm{H}_{3}{ }^{+}$particles have nearly identical momentum, so they should be equally effective at physical sputtering. Given these considerations, it is only possible to explain the great difference in the rate of carbon removal between hydrogen and helium by chemical processes, induced by hydrogen ions on the carbon surface. Such processes, as well as the role of EUV photons, are examined in detail in Section 5.

\subsection{Low-pressure SWD discharge plasma}

Even the simplest assessments, based on comparison of the basic discharge characteristics, demonstrate that the parameters of the plasma induced by EUV radiation are close to the parameters of a standard low-pressure discharge plasma, e.g. those used in microelectronics for the purpose of surface etching and cleaning. Thus, the removal of carbon due to EUV-induced plasma was compared to that of a low temperature discharge plasma to clarify the mechanisms of amorphous carbon removal.

The experimental setup is shown in figure 6 . The plasma is formed in a long quartz tube ( $100 \mathrm{~cm}$ long, $5.6 \mathrm{~cm}$ inner diameter) by means of a surface-wave discharge (SWD) at $81 \mathrm{MHz}$. RF excitation was achieved by two circular electrodes, placed close to one end of the tube. He or $\mathrm{H}_{2}$ was supplied to the tube end at a pressure 2.7 Pa. The 
power supplied to the discharge was between 20 to $50 \mathrm{~W}$, chosen to ensure that the plasma column extended from the electrode to the gas inlet, which served as a counter electrode for diagnostic measurements. The samples, which also served as an electrode to probe the plasma, were $<10 \times 10 \mathrm{~mm}^{2}$ in size, less than $10^{-3}$ of the counter electrode area.

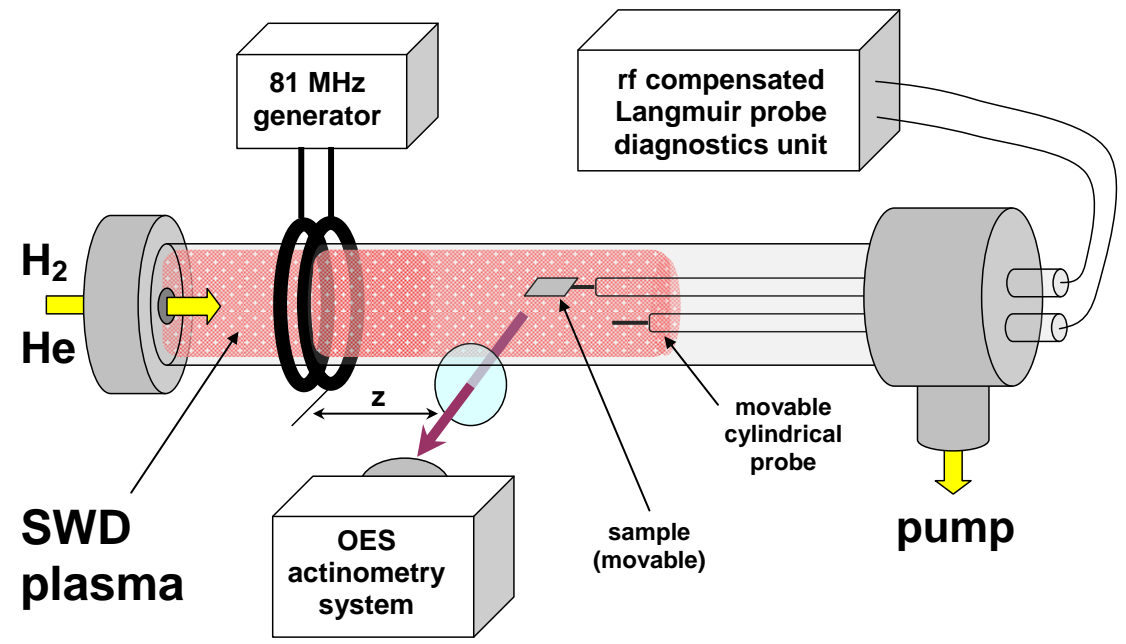

Figure 6. Set up for the experiments on C cleaning in SWD plasma, which has conditions similar to the conditions of EUV-induced plasma.

As is well known [23], the characteristic feature of the plasma column in surface-wave discharge is that the electron temperature and, accordingly, plasma potential and ion energy are nearly constant along the column. On the other hand, the plasma density and, accordingly, the ion flux incident on a surface, placed in the plasma, reduces with distance from the RF antenna. Therefore, the sample was placed on a travelling flat probe, able to move along the plasma column, thus, allowing the incident ion flux to be varied independently of the ion energy. To control the energy of the incident ions, the sample was biased with respect to the plasma 
potential. The sample was connected to a low-pass filter to remove the current induced by the plasma power supply. The parameters of the plasma above the sample surface were determined, based on measured sample voltage-current characteristics. Figure 7 shows typical VI characteristics of the sample in $\mathrm{He}$ and $\mathrm{H}_{2}$ at different distances from the RF antenna.

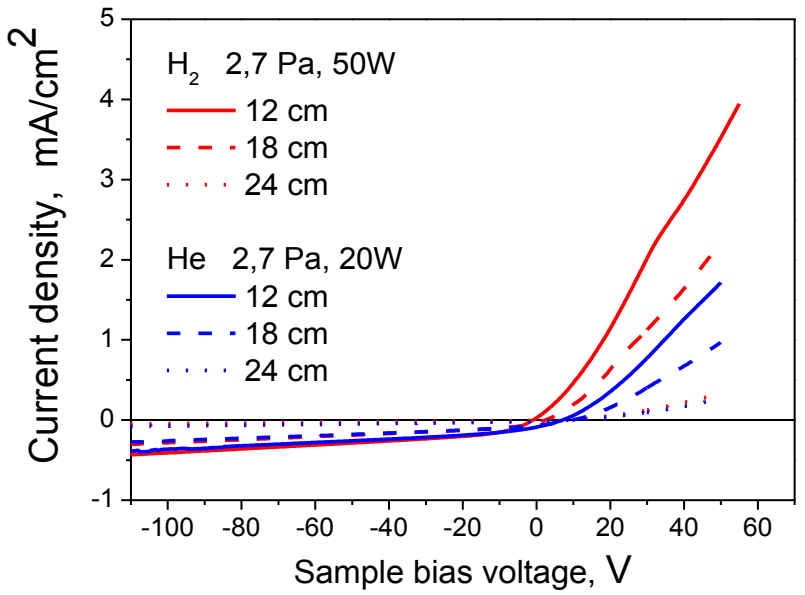

Figure 7. VI characteristics for the flat sample in SWD plasma column in $\mathrm{H}_{2}$ (solid lines) and $\mathrm{He}$ (dashed lines) at the different distances, $z$, from the RF antenna.

To verify the consistency of the measurements of the plasma parameters using the sample as a flat probe (and, as a result, the energy of ions on the sample surface), the experiment also used an rf-compensated standard cylindrical probe $(\sim 10 \mathrm{~mm}$ long, $\sim 0.1 \mathrm{~mm}$ in diameter). It should be noted that, compared with the "small" cylindrical probe, some distortion is observed in the electron part of the VI characteristics of the sample - the well-known electron depletion effect - that is quite expected for large-area probes. 
Therefore, the sample measurements are expected to underestimate the plasma density, which is extracted from the electron part of $\mathrm{VI}$ characteristics, while accurately estimating the electron temperature, $T_{e}$. At the same time, the plasma densities extracted from the ion part of VI characteristics of the cylindrical and the sample appear to be in a good agreement, although the definition of a "flat probe" for the sample cannot be applied exactly in this case because of the low plasma density. As was observed, the plasma sheath over the sample (probe) is rather big and not purely flat. Taking into account that this fact can lead only to small discrepancies, a "flat-probe" approach was used for calculating the energy and ion flux at the sample surface.

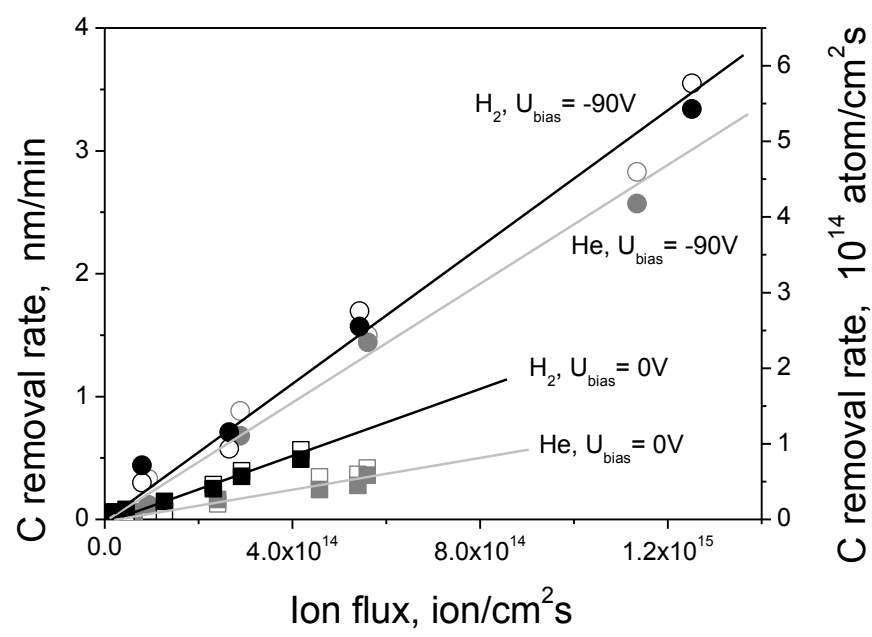

Figure 8. Carbon cleaning rate vs the ion flux (i.e. $\mathrm{H}_{3}^{+}$and $\mathrm{He}^{+}$for hydrogen and helium plasmas, respectively) at two (-90 $\mathrm{V}$ and $\mathrm{O} \mathrm{V}$ ) biases $\left(U_{\text {bias }}\right)$, applied to the sample. Data for hydrogen and helium are shown by black and grey colors, respectively. The lines are linear fits to the data. Open and filled symbols correspond, respectively, to XRF (EDS) and RS measurements of the carbon film thickness. 
As an example, figure 8 demonstrates the rate of carbon removal in hydrogen plasma (black symbols and lines) and helium plasma (grey symbols and lines) versus ion flux for different bias voltages $\left(U_{\text {bias }}=0\right.$ $\mathrm{V}$ and $\mathrm{U}_{\text {bias }}=-90 \mathrm{~V}$ ). With an estimated density of amorphous carbon film of $\sim 1.9 \mathrm{~g} / \mathrm{cm}^{3}$ [24], the rate of carbon removal was calculated in atom $/\left(\mathrm{cm}^{2} \mathrm{~s}\right)$ on the right scale. Open and filled symbols in figure 8 correspond to XRF (EDS) and RS data, respectively. The trend lines are the linear fits to the data. The slope of each line is interpreted as carbon removal probability or $\boldsymbol{C}$ yield per ion for the respective conditions (bias voltage and gas species).

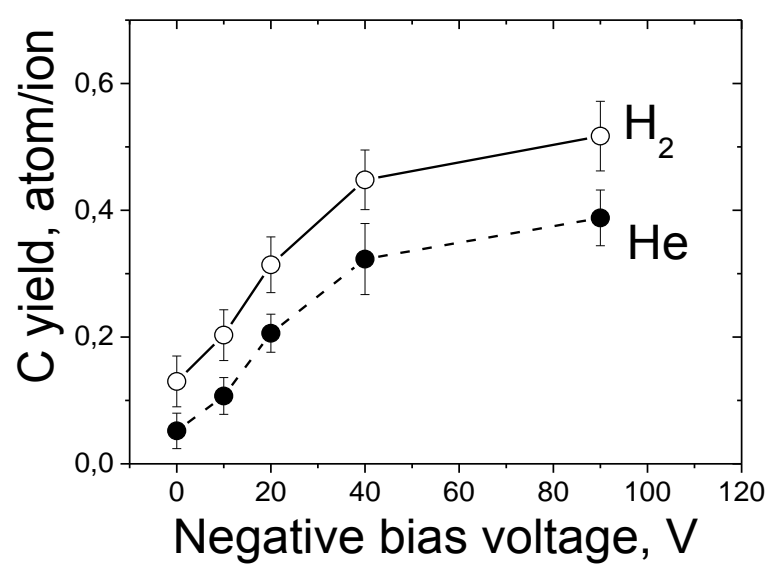

Figure 9. The $\mathrm{C}$ yield (atom/ion) versus the negative bias applied to the sample in $\mathrm{H}_{2}$ (open circles) and $\mathrm{He}$ (filled circles) SWD plasmas.

The $\mathrm{C}$ yield for both $\mathrm{H}_{2}$ and He plasma is shown in figure 9 as function of the negative bias applied to the sample. The error bars on vertical axis correspond, mainly, to the uncertainties in the measurements and calculations of the $C$ cleaning rate. As can be seen in figure 9, the $\mathrm{C}$ yield is higher for $\mathrm{H}_{2}$ plasma than for $\mathrm{He}$ plasma, and this difference increases with increasing bias. It should be noted that this plot also differs from the similar plot for EUV- 
induced plasma (compare with figure 5). It shows that, while the average plasma conditions are similar, the characteristics of EUVinduced plasma are dominated by the time-varying flux and intensity of ionizing radiation, incident on the carbon surface. A comparative analysis of the carbon removal mechanism in discharge plasma and EUV-induced plasma allows the role of the EUV radiation to be understood.

\subsection{Discussion}

To understand the detailed mechanism of C cleaning in an EUVinduced plasma and to characterize the role of EUV photons in this process, the EUV-induced and discharge plasmas should be compared in a physically clear manner. The role of EUV radiation in C-cleaning can be understood by comparing C-cleaning measurements with and without EUV radiation, but with similar fluxes and energy of the incident ions. For this purpose, we will try to find a relation between the ion energy, the applied bias, and plasma parameters in both types of plasmas.

The energy spectrum of the ions incident on the sample surface from the almost collision-less plasma sheath should have a notable peak. The peak of the ion energy spectrum and the peak width can be estimated as $\sim U_{\text {bias }}+U_{p}$ and $\sim 2 U_{f}$, respectively, where $U_{p}$ and $U_{f}$ are plasma and float potentials. In reality, the peak of the ion energy spectrum is somewhat lower, and the ion energy distribution is wider than given by this simple estimation. However, the validity of such a treatment has been confirmed by Monte Carlo calculations of the ion energy spectrum above sample surface [25]. Model calculations and estimates show that, because of the fast reaction: $\mathrm{H}_{2}^{+}+\mathrm{H}_{2} \rightarrow \mathrm{H}+\mathrm{H}_{3}{ }^{+}$ $\left(\mathrm{k}>10^{-9} \mathrm{~cm}^{3} / \mathrm{s}\right.$ for $\mathrm{T}_{\mathrm{i}}<1 \mathrm{eV}$ in the plasma volume), and the given 
experimental conditions, $\left(\mathrm{H}_{2}\right.$ pressure $2.7 \mathrm{~Pa}$, plasma density $10^{9}$ $\left.\mathrm{cm}^{-3}\right) \mathrm{H}_{3}{ }^{+}$is the dominant ionized species. [26].

In contrast to the discharge plasma, the type of ion and ion energy spectrum cannot be easily predicted using only qualitative considerations for the EUV-induced plasma. According to model calculations [25,26], $\mathrm{H}_{3}{ }^{+}$is still the dominant ion, but the energy distribution of both ions $\left(\mathrm{H}_{3}{ }^{+}\right.$and $\left.\mathrm{H}_{2}{ }^{+}\right)$is noticeably wider than the energy distribution of the ions in the continuous SWD plasma. In addition, the peak of ion energy distribution is a factor of two lower compared to that of the SWD. case energy equal to 0,5 U bias.

In experiments with the discharge plasma, the carbon removal yield per ion is assessed directly from the data (see above). For the pulsed EUV-induced plasma, the yield is calculatedas the average number of removed carbon atoms per EUV pulse divided by number of ions incident on the sample surface per pulse:

$$
R_{C}=\frac{N_{C}}{N_{i}}=\frac{\rho \cdot S \cdot\langle h\rangle \cdot e}{m_{C} \cdot Q_{i}}
$$

Where $\rho \approx 1.9 \mathrm{~g} / \mathrm{cm}^{3}$, is the carbon density, $S$ is the area of the focus, $\langle h\rangle$ is a spatial average of the thickness of the removed carbon layer, estimated from the measured $C$ layer thickness profile inside diaphragm. The charge is of the ion is $e_{,} m_{c}$ is the mass of the carbon atom, and $Q_{i}$ is the total number of ions incident on the sample surface. $Q_{i}$ was determined by integrating the ion current.

C cleaning yields, calculated for both discharge and EUV-induced for hydrogen (a) and helium (b) plasmas, versus the peak ion energy, calculated as described above, are shown in figures 10a and 10b, respectively. As was mentioned above, the error bars shown on the 
horizontal axis represent the approximate width of the ion energy distributions for both plasmas, while the error bars on vertical scale correspond to the uncertainties associated with measurements of the $C$ yield. For helium, the carbon yields for EUV-induced and SWD plasmas are rather similar, while for hydrogen, the two are quite significantly different. This shows that the mechanism of carbon removal in helium plasmas is the samefor both SWD and EUVinduced plasmas, and the role of EUV radiation is insignificant. For the hydrogen plasma, the differences show that the EUV radiation is inducing additional processes that increase the cleaning rate beyond a simple combination of physical and chemical sputtering.

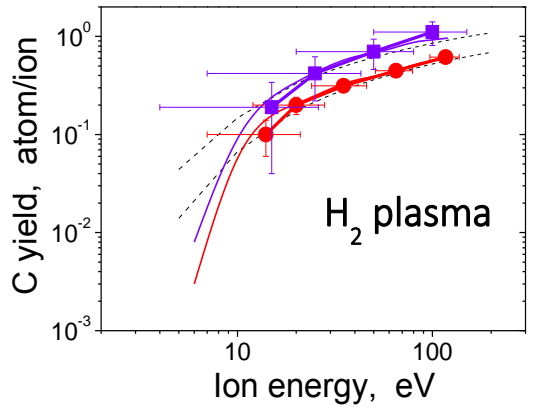

(a)

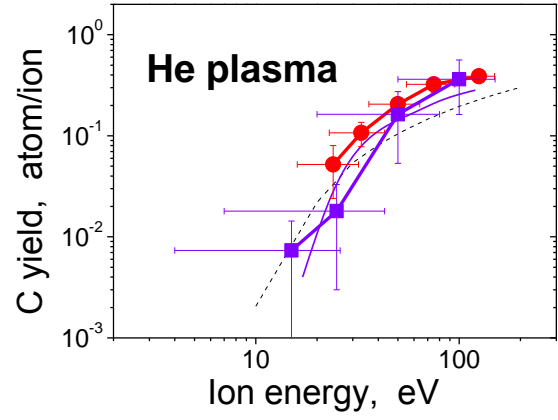

(b)

Figure 10. C atom yield (atom/ion) vs the mean energy of ions incident on the amorphous carbon surface in $\mathrm{H}_{2}$ (a) and $\mathrm{He}$ (b) plasmas. Big dark circles with thick lines between them are experimental data for SWD plasma while big dark squares with thick lines between them are experimental data for EUV-induced plasma. Dotted black lines are fits according to expression (1) with parameters from table 1 and 2. Solid thin lines are estimates made for $\mathrm{H}_{3}{ }^{+}$and $\mathrm{He}^{+}$ions by using TRIM calculations with parameters presented in table 3. 
Given the inert nature of helium ions, the removal of carbon from the surface is most likely due to physical sputtering only. Physical sputtering has been studied in the literature for virtually all elements of the periodic table, in both neutral and ionized states. Sputtering (sputtering yield) depends on the mass, charge, and energy, $E_{i}$, of the incident ions, as well as the surface binding energy, $E_{s}$, of the target atoms, etc. An accurate model of physical sputtering and resulting sputtering yield calculations can be found in [27]. In this review, a semi-empirical expression for the sputtering yield for almost all combinations of incident ion and target material was obtained, which gives a limited set of free parameters to fit in order to achieve reasonable agreement between observed and calculated sputtering yields Bohdansky et al. [28] Yanamura et al. [29]. The sputtering yield for light ions is given by:

$$
Y\left(E_{i}\right) \approx \frac{A}{E_{s}} \cdot \frac{S_{n}(\varepsilon)}{1+B \varepsilon^{0.3}} \cdot\left(1-\left(\frac{E_{t h}}{E_{i}}\right)^{1 / 2}\right)^{2.5}
$$

where $A, B$ are empirical coefficients, for which we used values obtained by Yamamura and Tawara [29]. $E_{\text {th }}$ is the threshold energy, which is taken as a free parameter. $S_{n}(\varepsilon)$ is the nuclear stopping cross-section, which can be expressed in terms of the elastic crosssection, $s_{n}(\varepsilon)$, and can be found by substituting a reduced ion energy, $\varepsilon_{\text {, }}$ into the analytical expression:

$$
s_{n}(\varepsilon) \approx \frac{3.441 \sqrt{\varepsilon} \ln (\varepsilon+2.718)}{1+6.355 \sqrt{\varepsilon}+\varepsilon(-1.708+6.882 \sqrt{\varepsilon})}
$$


The values of the parameters in equation (1), obtained from ref [29] for carbon surface bombarded by $\mathrm{He}^{+}$and $\mathrm{H}_{3}{ }^{+}$ions, are presented in table 1.

Table 1. The parameter values for equation $\mathbf{1}$ for
\begin{tabular}{ccc}
$\mathbf{H e}^{+}-\mathbf{C}$ and $\mathbf{H}_{\mathbf{3}}+\mathbf{-} \mathbf{C}$ sputtering according to[29]. \\
\hline & $\begin{array}{c}\text { Physical ion } \\
\text { sputtering }\left(\mathrm{He}^{+}\right)\end{array}$ & $\begin{array}{c}\text { Physical ion } \\
\text { sputtering }\left(\mathrm{H}_{3}^{+}\right)\end{array}$ \\
\hline$A$ & 0.0342 & 0.0407 \\
$B$ & 0.335 & 0.357 \\
$S_{n}(\varepsilon)$ & $115.03 \cdot \mathrm{S}_{\mathrm{n}}(\varepsilon)$ & $49.05 \cdot \mathrm{S}_{\mathrm{n}}(\varepsilon)$ \\
$\varepsilon$ & $9.2 \cdot 10^{-5} \cdot \mathrm{E}_{\mathrm{i}}$ & $2.09 \cdot 10^{-4} \cdot \mathrm{E}_{\mathrm{i}}$ \\
$E_{t h}$ & $3.87 \cdot \mathrm{E}_{\mathrm{s}}$ & $3.79 \cdot \mathrm{E}_{\mathrm{s}}$
\end{tabular}

As follows from expression (1), the most important parameter for the mechanism of physical sputtering is the binding energy of the target atoms: amorphous carbon in our case. The binding energy also determines the threshold energy, $E_{t h}$. As an estimate, $E_{s,}$ is usually assumed to be equal to the sublimation energy (enthalpy of vaporization $\underline{\Delta H_{C}}$ ) of the target material, which, for graphite-like surfaces, is $\Delta H_{C} \approx 7.43 \mathrm{eV}$. However, as shown [30,31] in the analysis of amorphous carbon sputtering by light ions, experimental data are well described using a smaller value of $E_{s} \approx 4.5 \mathrm{eV}$.

Therefore the expression (1) was fitted (dashed lines in figures 10a and $10 \mathrm{~b}$ ) to the experimental data for both SWD and EUV-induced plasmas, using the parameters from table 1 , and taking $E_{s}$ and $E_{\text {th }}$ as 
free parameters. The values of $E_{s}$ and $E_{t h}$ for $\mathrm{He}^{+}$(see table 2) were found to be even lower than those found in ref [30,31], which can be clearly seen in figure $10 \mathrm{a}$ and $10 \mathrm{bwhen}$ considering the $\mathrm{C}$ sputtering yield at low ion energies.

Rigorous methods, that directly model the interaction of ion beams with a surface, such as TRIM (TRansport of Ions in Matter) were also used to understand the carbon cleaning mechanism. TRIM is a Monte Carlo computer program that calculates the interactions of energetic ions with amorphous targets. The threshold energy, $E_{t h}$, for sputtering amorphous materials is usually found to be close to the energy required to displace a target atom $E_{\text {displ }}=25 \mathrm{eV}$ (see table 3). Although TRIM is not optimum for non-monochromatic ion-energy spectra, by approximating the ion-energy spectrum by its average value, the rate of ion sputtering of carbon films by $\mathrm{He}^{+}$and $\mathrm{H}_{3}{ }^{+}$ions was estimated, and used to gain insight into the mechanism of the carbon removal. $\mathrm{H}_{3}{ }^{+}$ions were approximated by two different methods: $\mathrm{H}_{3}{ }^{+}$ions were assumed to be a hydrogen ion with a mass of $3 \mathrm{amu}$, and, in the second case, as a helium ion with a mass of $3 \mathrm{amu}$. The results for these two cases were found to differ by less than $1 \%$. As above, the density of the carbon target is taken to be $1.9 \mathrm{~g} / \mathrm{cm}^{3}$. Three parameters: $E_{\text {displ, }} E_{\text {bin.lat. }}$ and $E_{\text {bin.surt }}$ were varied to obtain a best fit (solid lines) to the data in figures 10a and 10b. $E_{\text {bin.lat. }}$ is the binding energy of non-surface carbon atoms in the layer, while $E_{\text {bin.surt }}$ is the surface binding energy of carbon atoms. The values of the TRIM calculation parameters are presented in table 3. As can be seen from the disagreement between the best-fit values obtained from TRIM and those reported in the literature (see table 3), the removal of the amorphous carbon in an EUV-induced plasma cannot be described by kinetic sputtering. For the case of $\mathrm{He}$, this is not surprising, since physical sputtering is the only available mechanism and the EUV pulse simply generates the plasma to initiate sputtering. 
Table 2. Parameters in fitting (1) used for the three data sets in figures

$10 \mathrm{a}$ and $10 \mathrm{~b}$.

\begin{tabular}{cccc}
\hline & $\begin{array}{c}\text { Physical ion } \\
\text { sputtering } \\
\left(\mathrm{He}^{+}\right)\end{array}$ & $\begin{array}{c}\text { Chemical ion } \\
\text { sputtering } \\
\left(\mathrm{H}_{3}^{+}\right)\end{array}$ & $\begin{array}{c}\text { RIE (EUV) + chemical ion } \\
\text { sputtering }\left(\mathrm{H}_{3}^{+}\right)\end{array}$ \\
\hline $\mathrm{E}_{\mathrm{th}}(\mathrm{eV})$ & 7.35 & 2.27 & 1.52 \\
$\mathrm{E}_{\mathrm{s}}(\mathrm{eV})$ & 1.9 & 0.6 & 0.4 \\
\hline
\end{tabular}

Table 3. TRIMmodel parameters used for the three data sets in figures $10 \mathrm{a}$ and $10 \mathrm{~b}$.

\begin{tabular}{ccccc}
\hline & $\begin{array}{c}\text { Physical ion } \\
\text { sputtering }\left(\mathrm{He}^{+}\right)\end{array}$ & $\begin{array}{c}\text { Chemical ion } \\
\text { sputtering }\left(\mathrm{H}_{3}{ }^{+}\right)\end{array}$ & $\begin{array}{c}\text { Recommended by } \\
\text { TRIM (graphite) }\end{array}$ & $\begin{array}{c}\text { RIE (EUV) }+ \\
\text { chemical ion } \\
\text { sputtering }\left(\mathrm{H}_{3}{ }^{+}\right)\end{array}$ \\
\hline$E_{\text {displ. }}$ & 15 & 4.5 & 25 & 4.5 \\
$E_{\text {bin.lat. }}(\mathrm{eV})$ & 2 & 2 & 3 & 2 \\
$E_{\text {bin.surf. }}(\mathrm{eV})$ & 4.5 & 3 & 7.37 & 1.4 \\
\hline
\end{tabular}

As follows from (1) and TRIM calculations, the removal of carbon in the discharge $\mathrm{H}_{2}$ plasmahas a similar character to the removal of $\mathrm{C}$ by $\mathrm{He}^{+}$ions, but the $\mathrm{C}$ yield is higher. However, compared to $\mathrm{He}^{+}$ions, agreement with experiment data can only be achieved with a substantial reduction of the threshold energy (see tables 2and 3).For example, the TRIM calculation shows that the displacement energy is almost equal the surface binding energy. This is usually interpreted as the possibility of a director ion-stimulated reaction between implanted ions (atoms) with the displaced ("free") target atoms 
[32].This mechanism of removal of target atoms by low-energy ions, commonly called chemical sputtering, is well known for $\mathrm{H}$ sputtering of carbon [30-32].

The penetration depth of low-energy hydrogen ions (with energies of tens of $\mathrm{eV}$ ) in carbon is small enough ( $\leq 3 \mathrm{~nm}$ ) to allow the resulting volatile species to diffuse to the surface and escape (mainly $\mathrm{H}_{2}$ and $\left.\mathrm{CH}_{4}\right)$ [33].

Atomic hydrogen, formed in plasma due to gas dissociation, may promote carbon removal. However, the flux of atomic hydrogen, incident on the sample surface, should be small in both the EUVinduced and SWD plasmas. For example, the estimates of the density of $\mathrm{H}$ atoms in the discharge tube, from actinometry on argon atoms [20], showed that the relative concentration of atomic hydrogen was $\sim 0.5-1 \%$. According to the results of [30], and the measured ratio of hydrogen ionic and atomic fluxes, incident on the sample is such that the rate of the reactive ion etching (RIE) involving hydrogen atoms should not exceed the rate of chemical sputtering for SWD hydrogen plasmas.

Indeed, in the EUV-induced hydrogen plasma, the C atoms yield per ion is higher than in the SWD plasma. To obtain agreement between experimental results and calculations using only physical sputtering, the energy threshold has to be made very small (much smaller than any physical arguments would allow). Additionally, it should be expected that the energy threshold is independent of the plasma generation mechanism. The difference between the EUV-induced and the discharge plasmas in $\mathrm{H}_{2}$ is relatively constant for all ion energies, which offers indirect evidence that this difference is due to the EUV pulse, since the EUV photon fluence is the same for all data plotted in figures 10a and 10b. This increase in the yield of carbon 
for EUV-induced plasma can be explained only by an additional chemical mechanism, i.e. removal by the additionally formed atomic hydrogen.

This is also indirectly confirmed by TRIM calculations, in which, to describe the carbon yield in the EUV-induced hydrogen plasma, $E_{\text {bin.surf }}$ has to be reduced practically to an unrealistically low value (see table 2). According to [30,31] this can only be due to ionstimulated reactions between hydrogen and carbon on the surface. This process is analogous to the process of RIE [30] with the difference that the atomic hydrogen is formed directly on the surface layer of carbon, due to the dissociation and ionization of adsorbed molecular hydrogen, followed by the dissociated species being "dissolved in the surface layer." Furthermore, the EUV photons that are absorbed in the carbon generate excited species (carbon radicals and ions). At the carbon layer surface, we estimate from reflection loss data and secondary electron yield data [34] that up to $4 \%$ of the surface carbon atoms will be either ionized or form a radical. We will refer to this combination of processes as reactive ion sputtering (RIS).

In a sense, the process of the formation of atomic hydrogen on the surface is similar to the process of surface contamination and oxidation of surfaces. As is known, these processes are caused by dissociation of hydrocarbons and water during exposure to EUV radiation [2-7]. Moreover, the oxidation of carbon contamination under EUV radiation is even observed in ultra-high vacuum.

To estimate if surface dissociation is significant, the surface coverage of molecular hydrogen must be estimated. The energy of desorption and residence timefor hydrocarbon molecules and hydrogen molecules are very different. For polyatomic hydrocarbon molecules, 
the desorption energy is much higher than that of hydrogen. Therefore, even with large concentration differences in the volume above the sample,the surface concentrations of hydrogen and hydrocarbons may be comparable. These considerations allow the desorption energy of $\mathrm{H}_{2}$ from an amorphous carbon surface during EUV illumination to be estimated.

Assuming that the increase in the carbon yield in the EUV-induced plasma is due only to the EUV-dissociated hydrogen (i.e. almost half of the carbon is removed by RIS), the removal of one $C$ atom is estimated to require approximately $10^{3}$ EUV photons. If it is also assumed that RIS results in $\mathrm{CH}_{4}$, the minimum fraction of the surface occupied by dissociated $\mathrm{H}_{2}$ molecules can be estimated to be $\theta \approx$ $2 \cdot 10^{-3}$. Sorption equilibrium coverage is given by:

$$
H_{2}+\text { surface } \underset{v_{d}}{\stackrel{k}{\leftrightarrow}} H_{2}^{\text {surface }} \quad \theta=\frac{k\left[H_{2}\right]}{v_{0} e^{-\frac{E_{d}}{k T}}}
$$

(where $\left[\mathrm{H}_{2}\right] \sim 710^{14} \mathrm{~cm}^{-3}(3 \mathrm{~Pa}), \mathrm{k} \sim 10^{-10} \mathrm{~cm}^{3} / \mathrm{s}$ is the rate constant corresponding to the sticking coefficient $\sim 1$ and, in fact, is the rate constant of collisions in gas phase, $v_{0} \sim 10^{13} \mathrm{~s}^{-1}$ is the so-called "attempt frequency", that can be interpreted as the vibrational frequency of the adsorbed species in a potential well with a depth corresponding to that of the desorption energy, $E_{d}$, so that only the fraction of the particles having an energy higher than $E_{d}$. From eq. (3), $E_{d} \sim 380 \mathrm{~K}$ for surface adsorbed atomic hydrogen (ignoring atomic hydrogen "dissolved" in the surface layer). Nevertheless this estimate corresponds to the desorption energy of molecular hydrogen measured on different materials, both metals [20] and dielectrics [35]. It should be noted that the density of adsorption sites is correlated with surface defects, rather than simply the density of 
surface atoms. In this sense, the estimated value of $E_{d}$ characterizes not only the surface but also the depth of $\mathrm{H}_{2}$ diffusion [33]. Therefore, it reflects a certain characteristic binding energy of $\mathrm{H}_{2}$ molecules with the surface layer of carbon, part of which is activated by EUV radiation and the plasma. This "activated"(chemically active) carbon in principle is able to react with $\mathrm{H}_{2}$, while atomic hydrogen is able to react with carbon whether it is activated or not.

Thus, under EUV radiation, carbon removal is not only due to physical and chemical sputtering by ionized species, but is also due to the creation of volatile species from reactions between radicals and between radicals and molecular species. Since radicals are not influenced by bias voltages, there is very little atomic hydrogen incident on the surface. Instead, the radicals are created by the dissociation of both the carbon surface, and adsorbed molecular hydrogen.

\subsection{Conclusion}

In the given work, the removal of thin amorphous carbon films from Si substrates in plasmas in presence of two types of gas: helium and hydrogen, was studied. The experimental data clearly show that the measured carbon removal rates for SWD and EUV-induced He plasmas are similar. He removes carbon through physical sputtering. Hydrogen was found to work differently. It was shown that the hydrogen cleaning mechanism in the case of an EUV-induced plasma is about 5 times more efficient then in a SWD plasma. The qualitative analysis showed that the rate of cleaning in EUV-induced $\mathrm{H}_{2}$ plasma is greater than expected owing to reactive ion etching by atomic hydrogen, adsorbed on the surface of carbon film. Analytical and computational models were used to show that the data do not support physical sputtering in the case of hydrogen plasmas. Yet, a 
consistent carbon binding energy and threshold energy for both SWD and EUV induced plasmas could be found once chemical sputtering is taken into account. However, an EUV plasma was found to create a larger ratio of atomic versus ionic hydrogen on the sample surface than in the case of SWD plasma. 


\section{References.}

1. International Technology Roadmap for Semiconductors, http://www.itrs.net

2. Boller K J, Haelbich R P, Hogrefe H, Jark W, and Kunz C 1983 J Nucl. Instrum. Methods Phys. Res. B 208 273-279.

3. Meiling $H$, Mertens B, Stietz F, Wedowski M, Klein R, Kurt R, Louis E, and Yakshin A E 2001 Proc. of SPIE 4506 93-104.

4. Hollenshead J and Klebanoff L 2006 J. Vac. Sci. Technol. B 24 6482.

5. Matsunari S, Aoki T, Murakami K, Gomei Y, Terashima S, Takase $H$, Tanabe M, Watanabe $Y$, Kakutani $Y$, Niibe $M$, and Fukuda $Y$ 2007 Proc. of SPIE 6517 65172X-8.

6. Koster N, Mertens B, Jansen R, van de Runstraat A, Stietz $F$, Wedowski M, Meiling H, Klein R, Gottwald A, Scholze F, Visser M, Kurt R, Zalm P, Louis E, andYakshin A 2002 Microelectron Eng 612 65-76.

7. Chen J Q, Louis E, Lee C J, Wormeester H, Kunze R, Schmidt $H$, Schneider D, Moors R, van Schaik W, Lubomska M, and Bijkerk F 2009 Optics Express 17 16969-16979.

8. Bienert M, Göhnemeier A, Natt O, Lowisch M 2009 J. Micro/Nanolith. 8-4 041509.

9. Banine V, Benschop J 2004 Proc. of SPIE 54011.

10. Silverman P J 2005 J. Microlith., Microfab., Microsyst. 4(1) 011006.

11. Seisyan R P 2005 Techn. Phys. 50-5 535-545.

12. Motai K, Oizumi H, Miyagaki S, Nishiyama I, Izumi $A$, Ueno $T$, and Namiki A, 2008 Thin Solid Films 516 839-843.

13. Oizumi H, Izumi A, Motai K, Nishiyama I, and Namiki A, 2007 Jap. J App. Phys Part 2-Letters \& Express Letters 46 L633-L635.

14. Graham S, Charles A S, Clift W M, Leonard E K, and Sasa B 2003 SPIE, 5037 460-469.

15. Tsarfati T, Zoethout E, R W E van de Kruijs, and Bijkerk F 2009 Surf. Sci 603 2594-2599. 
16. Oizumi $H$, Yamanashi $H$, Nishiyama I, Hashimoto $K$, Ohsono $S$, Masuda A, Izumi A, and Matsumura H, 2005 SPIE 5751 11471154.

17. Nishiyama I, Oizumi $H$, Motai $K$, Izumi $A$, Ueno $T$, Akiyama $H$, and Namiki A 2005 AVS 23 3129-3131.

18. Mertens B, Wolschrijn $B$, Jansen R, Koster N, Weiss M, Wedowski M, Klein R, Bock T, and Thornagel R 2003 SPIE 5037 95-102.

19. Braginsky $O V$, Kovalev A S, Lopaev D V, Malykhin E M, Rakhimova T V, Rakhimov A T, Vasilieva A N, Zyryanov S M, Koshelev K N, Krivtsun V M, van Kaampen M and Glushkov D 2012 J App. Phys. 111093304.

20. Zyryanov S M, Kovalev A S, Lopaev D V, Malykhin E M, Rakhimov A T, Rakhimova T V, Koshelev K N, and Krivtsun V M 2011 Plasma Phys. Rep. 37-10 881-889.

21. Malykhin E M, Krivchenko V A, Lopaev D V, Rakhimova T V and Zyryanov S M 2011 Moscow University Physics Bulletin 66-1 54-58.

22. Koshelev $\mathrm{K} \mathrm{N}$ et al. 2006 in EUV Sources for Lithography (Ed. V Bakshi) (Bellingham, Wash.: SPIE Press,) p. 175.

23. Moisan M, Pelletier J 1992 Microwave Excited Plasmas, Plasma Technology (Amsterdam-London-New York-Tokyo: Elsevier).

24. Nakayama T, Miyake A, Takase H, Terashima S, Sudo T, Watanabe $Y$ and Fukuda Y2009 SPIE 727172713.

25. Malykhin E, Lopaev D, Rakhimov A, Rakhimova T, Braginskii O, Kovalev A, Vasil'eva A and Zyryanov S M 2011 Moscow University Physics Bulletin 66-2 184-189.

26. Astakhov D, Goedhee W J, Lopaev D, Ivanov V, Yakushev O, Krivtsun V M, Koshelev K. and Bijkerk F 2012. 2D PIC modeling of the EUV induced hydrogen plasma and comparison to the observed to the observed carbon etching rate. International Workshop on EUV and Soft X-ray Sources: Dublin, Ireland (2012, oktober 08 - 2012, oktober 11).

27. Sigmund P 1969 Phys.Rev. 184383.

28. Bohdansky A 1984 J Nucl. Instrum. Methods Phys. Res. B 2587.

29. Yamamura Y and Tawara H1996At.Data Nucl.Data Tables 62149. 
30. Hopf C, von Keudell A, and Jacob W 2003 J App. Phys. $942373-$ 2380.

31. Liu S, Sun J, Dai S, Stirner T, and Wang D 2010 J App. Phys 108 073302.

32. Bekrambekov L B 2008 Processes in solid under the ion and plasma irradiation (Moscow: M.MIPHI).

33. Moller W 1989 J Nucl. Materials 162-164 138-150.

34. Chen, J, E Louis, J Verhoeven, R Harmsen, C J Lee, M Lubomska, van Kampen M, van Schaik W, and Bijkerk F 2010 Applied Surface Science 257-2 354-361.

35. Kim Y C and Boudart M 1991 Langmuir 7 2999-3005. 


\title{
5. Characterization of carbon contamination under ion and hot atom bombardment in a tin-plasma extreme ultraviolet light source
}

\begin{abstract}
Molecular contamination of a grazing incidence collector for extreme ultraviolet (EUV) lithography was experimentally studied. A carbon film was found to have grown under irradiation from a pulsed tin plasma discharge. Our studies show that the film is chemically inert and has characteristics that are typical for a hydrogenated amorphous carbon film. It was experimentally observed that the film consists of carbon ( 70 at. \%), oxygen ( 20 at. \%) and hydrogen (bound to oxygen and carbon), along with a few at. \% of tin. Most of the oxygen and hydrogen are most likely present as $\mathrm{OH}$ groups, chemically bound to carbon, indicating an important role for adsorbed water during the film formation process. It was observed that the film is predominantly $\mathrm{sp}^{3}$ hybridized carbon, as is typical for diamond-like carbon. The Raman spectra of the film, under 514 and $264 \mathrm{~nm}$ excitation, are typical for hydrogenated diamond-like carbon. Additionally, the lower etch rate and higher energy threshold in chemical ion sputtering in $\mathrm{H}_{2}$ plasma, compared to magnetronsputtered carbon films, suggests that the film exhibits diamond-like carbon properties.
\end{abstract}

\section{Introduction}

The phenomenon of the contamination of optics under the action of EUV radiation is a known problem that is actively studied. Even in vacuum conditions, carbon atoms are deposited onto a surface when residual hydrocarbons dissociate during interaction with energetic photons [1]. The carbon atoms can then accumulate on 
surfaces, which is undesirable for reflective optics because the carbon layer absorbs radiation. For instance, EUV-induced carbon contamination of grazing incidence optics in synchrotron beam lines is one of the major reasons for the optics' reduced reflectivity. The study of this problem began a few decades ago [2], and several cleaning mechanisms have since been investigated [3-5].

Extreme ultraviolet lithography (EUVL) is currently the most advanced technology for the fabrication of integrated circuits with characteristic half-pitch $\leq 22 \mathrm{~nm}$. In modern $13.5 \mathrm{~nm}$ EUV lithography, the main contaminant on optics is carbon. Even at pressures as low as $10^{-5}-10^{-6} \mathrm{~Pa}$, the $\mathrm{C}$ film deposition rate can be as high as $0.01-1 \mathrm{~nm} / \mathrm{h}$ for an average EUV-radiation intensity of about 0.1-1 W/cm ${ }^{2}$. A carbon film will cause a reflectivity loss of about $1 \%$ /nm per mirror, which is especially critical for optical systems that have multiple reflecting surfaces.

Off-line removal of EUV-induced carbon contamination also reduces the duty cycle of EUVL, which is undesirable $[1,6,7]$, and ultimately, increases the operating cost of the EUVL process. However, with the use of fluxes of ions or neutral reactive species to restore reflectivity, the lifetime of a standard Mo/Si MLM system can be extended to several thousand hours [8]. A new cleaning strategy that uses the plasma, induced by EUV ionization of the low-pressure gas (usually $\mathrm{H}_{2}$ ) over the mirror surface, was recently proposed [9]. This inline cleaning process has the potential to increase the duty cycle of EUVL.

Until recently, magnetron deposited carbon was frequently used as a model for EUV-induced carbon growth. However, the characteristics of a carbon film depend on the deposition conditions. In practice, this means that the optics located close to the EUV light source (i.e., collector mirrors) could be coated with a significantly denser carbon 
film, due to the presence of a broad spectrum of high energy photons and ions. The structure of EUV-induced carbon films determines the reflectivity losses of the mirror. For instance, the denser structure of diamond-like carbon (DLC) film leads to more EUV absorption compared to a polymeric layer or "soft" amorphous carbon layer of the same thickness. Furthermore, because the carbon in DLC films is more tightly bound, the films are likely to be more difficult to clean. Hence, it is necessary to know how the phase of carbon may vary depending on the growth conditions.

In the presence of water and heavy hydrocarbons, a carbon film is likely to be hydrogenated. Typically, hydrogenated amorphous carbons are classified into four classes, illustrated by the phase diagram shown in figure 1 [10].

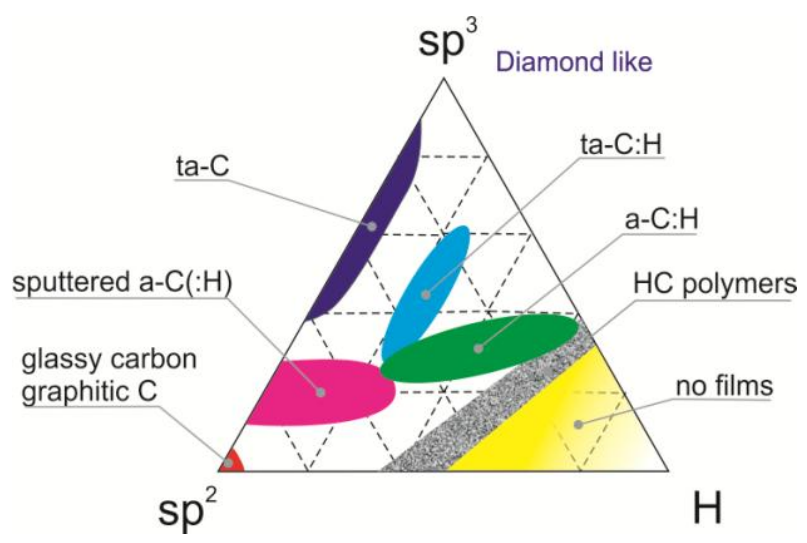

Figure 1. Phase diagram of hydrogenated amorphous carbon films.

All a-C:H films have a specific hydrogen content and properties that are revealed by applying a combination of different diagnostic techniques, such as X-ray photoelectron spectroscopy (XPS), Raman spectroscopy (RS), energy-dispersive X-ray (EDX) spectroscopic 
analysis, electron energy loss spectroscopy (EELS), transmission electron microscopy (TEM), and others.

In this paper, we report the experimental study of carbon-based films, deposited on the collector mirror of an EUV source. The condition under which the film was grown will be described, as well as analysis showing that the film has DLC characteristics.

\subsection{Experiment}

\subsubsection{Film deposition}

The experimental set up is schematically shown in figure 2. A Z-pinch discharge in Sn vapour is used as a source of pulsed EUV emission. The discharge is initiated using an Nd:YAG laser pulse to evaporate tin from the surface of a liquid tin cathode. The Sn vapour short circuits the $3 \mathrm{~mm}$ gap to the anode, which is biased at high voltage (3-4 kV). A few $\mathrm{nH}$ inductance of the discharge circuit allows a highly ionized $\left(\mathrm{Sn}^{8+-11+}\right)$ plasma to be produced, which with further pinching leads to a powerful shot of EUV emission being generated. The energy input into the discharge is 1-2 J, while CE (conversion efficiency) to $13.5 \mathrm{~nm}$ emission in a $2 \%$ spectral band is about $1 \%$. The shot repetition rate of the EUV source is $1.5 \mathrm{kHz}$, which allows a high photon fluence for a period of $\sim 3$ hours with 3-5\% stability.

The Z-pinch emission is collected by a set of grazing incidence cylindrical Mo mirrors, called the EUV collector. Our test bench collector consists of six mirrors, located at a distance of $48 \mathrm{~cm}$ from the source of radiation. Each mirror consists of a curved glassceramic substrate with an ultra low coefficient of thermal expansion (sital) that is $45 \mathrm{~mm} \times 60 \mathrm{~mm}$ square, and has a thickness of $6 \mathrm{~mm}$. 
High reflectivity EUV range is achieved by coating the substrate with $50 \mathrm{~nm}$ of Mo.

To protect the collector from the products of the Z-pinch discharge, a series of traps are located between the discharge and the mirrors. The debris mitigation system consists of a magnetic field to defect most ions with an energy less than $100 \mathrm{keV}$ and rotating foil trap, which effectively collects all micron-sized tin droplets moving at speeds of less than $500 \mathrm{~m} / \mathrm{s}$. Thus, the collector is exposed to radiation (both in-band EUV, and deep ultraviolet), high energy ions, and high velocity $\mathrm{Sn}$ debris during source operation.

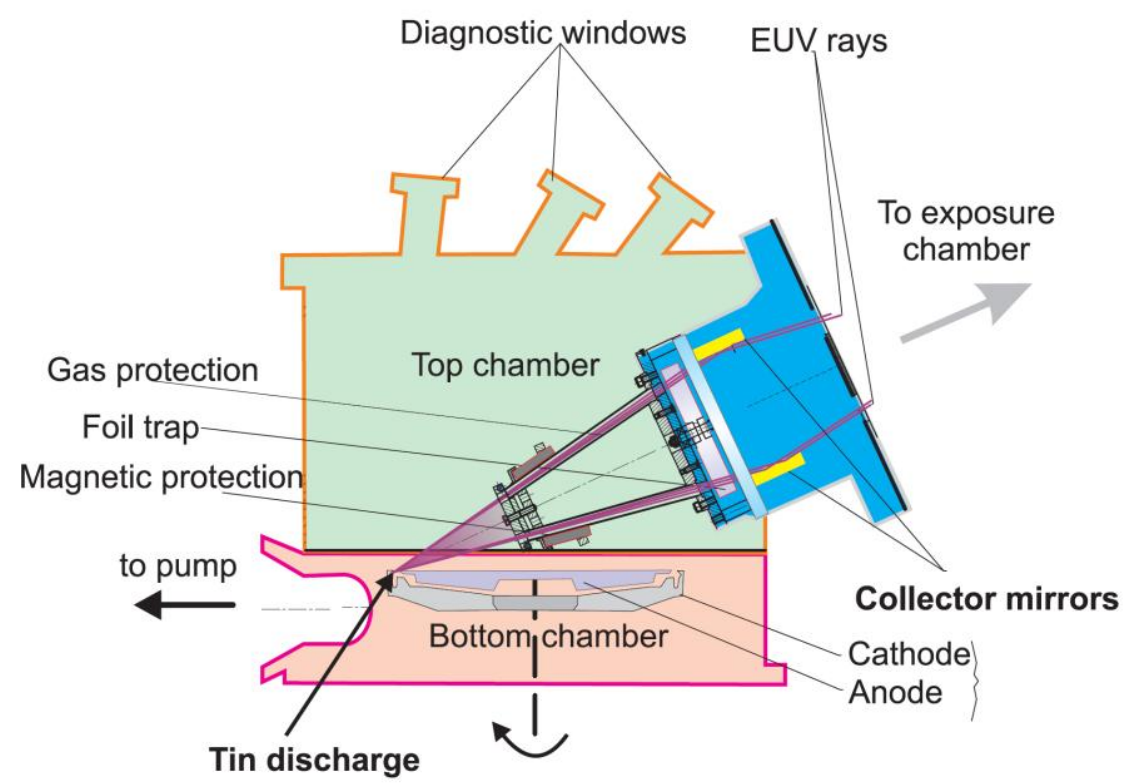

Figure 2. EUV tin plasma source for direct reproducing EUV-lithography tool conditions.

Before the experiments, the source chamber was evacuated to 3$5 \cdot 10^{-6}$ Torr. The partial pressures of the background gases were: 
water $\sim 10^{-7}$ Torr, nitrogen $\sim 10^{-7}$ Torr oxygen $\sim 10^{-9}$ Torr, and residual hydrocarbons $10^{-9}$ Torr. The experimental set up is schematically shown in figure 2. During operation, $\mathrm{H}_{2}$ is fed into the vacuum channel, where the collector mirrors are located. The hydrogen flows in the direction of the EUV source chamber, slowing down and scattering energetic ions and small Sn droplets in collisions with neutral and charged particles of $\mathrm{H}_{2}$ plasma formed in the collector optics channel. The presence of $\mathrm{H}_{2}$ over the collector mirrors notably decreases the contamination rate of the mirrors by carbon. The $\mathrm{H}_{2}$ pressure in the channel is $\sim 10^{-3}$ Torr and does not influence on EUV source operation [11].

\subsubsection{Analysis}

Our contamination film was gray-brown in color, and transparent in the visible region. The films, which were still attached to the collector mirror, were placed in $65 \% \mathrm{HNO}_{3}$ acid. It was noticed that, even after the underlying molybdenum was etched away ( 20 seconds) with nitric acid, the carbon film remained on the sital substrate. The undamaged carbon film flakes were then removed from the substrate and transferred to a copper support grid with a $1 \mathrm{X} 1 \mathrm{~mm}$ mesh, and then sandwiched in a slotted copper envelop grid to provide mechanical stability.

The total thickness of the film was found to be of the order of $150 \mathrm{~nm}$ after few hundreds millions EUV shots. The thickness was measured by EELS, in the energy-filtered transmission electron microscopy (EFTEM) mode. At this thickness, a film of magnetron sputtered carbon would be expected to be completely etched away within 10 seconds in $65 \% \mathrm{HNO}_{3}$ acid. 


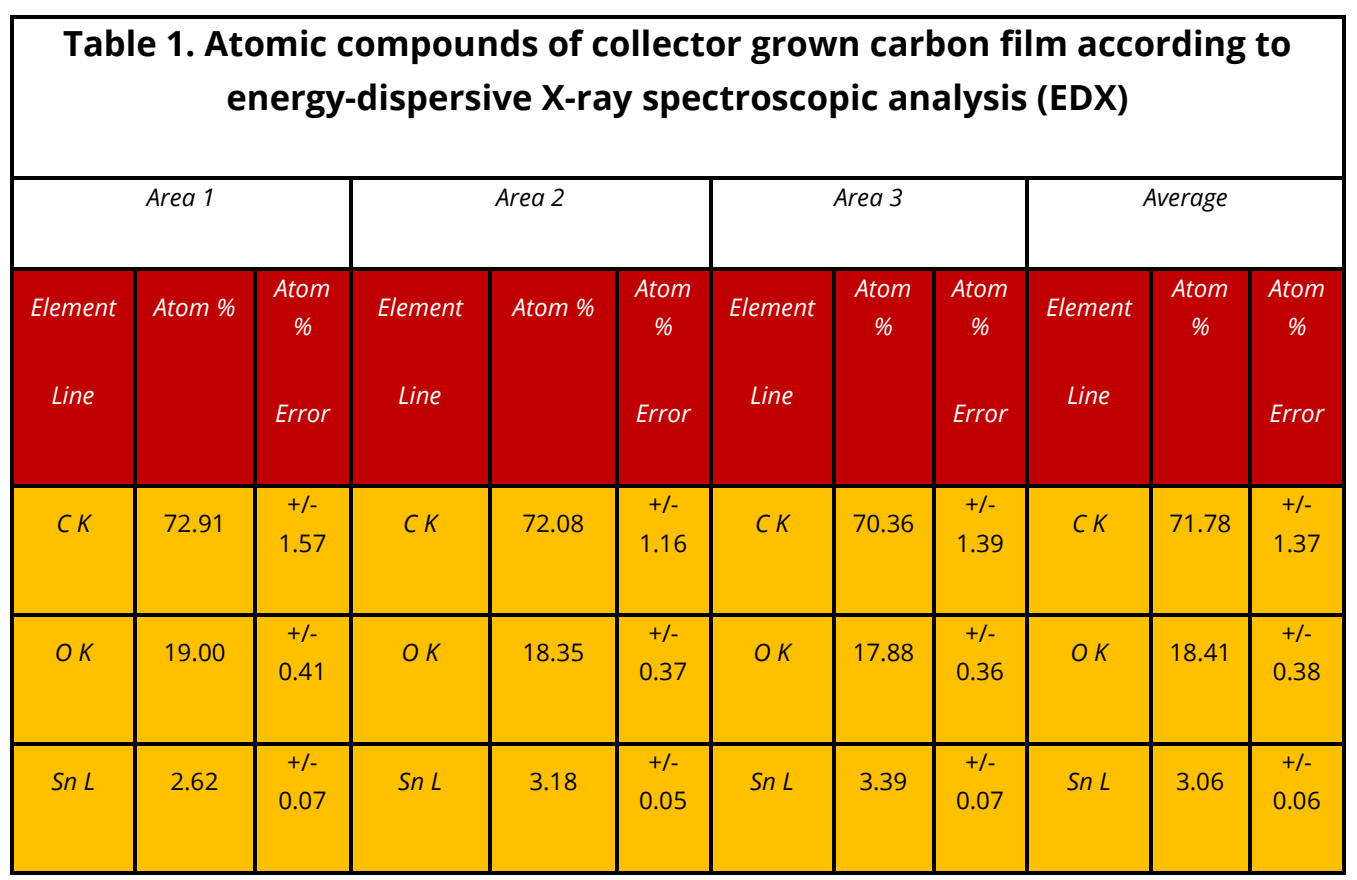

To understand the atomic composition of the film, EDX was used. According to EDX results (see table 1) the film is, on average, composed of the order of approx. 72 at. \% C, and approx. 18 at. \% O. In addition, trace elements from the EUV source fuel (Sn), electrodes (Fe, $\mathrm{Ni}$, and $\mathrm{Mg}$ ), and the collector mirror (Mo, and $\mathrm{Si}$ ) were also found. The sample was also slightly contaminated by the attempts to chemically etch it ( $\mathrm{Cr}, \mathrm{P}$, and others). Despite these low levels of various elements, the film is predominantly carbon, oxygen and tin, which is expected. We assume that the carbon and oxygen content of the film comes from background gases and adsorbed water.

Within the film, there are larger particles that are denser than the surrounding film. EELS spectra (data not shown) for the film are typical for amorphous carbon. Secondary electron microscopy (SEM), TEM, and high resolution TEM (HRTEM) images show the presence of submicron tin droplets. The large droplets are not randomly distributed. The droplet tracks are parallel to each other, indicating 
that they are ballistic tin droplets that have travelled directly through the rotating foil trap (part of mitigation protection system) and have a velocity more than $500 \mathrm{~m} / \mathrm{s}$ (the minimum speed to allow the particles to pass through the foil trap).

Interpretation of the HRTEM carbon lattice images gives fringes with d-spacings of 0.15 and $0.25 \mathrm{~nm}$ in the areas from 2 to $10 \mathrm{~nm}$. Such lattice structure are similar to ion-beam deposited carbon and carbon produced by shock synthesis designated as "0.25-i-carbon" and presented in [12]. Only in one case the lattice fringes yielding a $\mathrm{d}$-spacing of $0.205+/-0.001 \mathrm{~nm}$, associated with (111) cubic or $\mathrm{n}$ diamond lattice plane, were found. However, no evidence for the $0.178 \mathrm{~nm}$ and $0.126 \mathrm{~nm}$ d-spacings, associated with the (002) and (022) diamond lattice planes, were found.

Atomic force microscopy (AFM) measurements indicate that the surface is relatively rough, with an RMS roughness of a few tens of $\mathrm{nm}$.

More accurate information on the structural properties of carbon films was obtained with an XPS study (Thermo Theta Probe monochromated $\mathrm{Al} \mathrm{Ka}$ ). Figure 3 shows the characteristic $\mathrm{C}$ 1s peak from the carbon film. The atomic percent of carbon was determined to be $70 \pm 2 \%$, in agreement with the EDX data (tab 1). Since XPS is a surface sensitive technique, whereas EDX probes the complete $150 \mathrm{~nm}$ thick carbon film, similar composition indicates a homogeneous in-depth composition.

Deconvolution was carried out after subtracting the background by Shirley's method [13]. The peaks were deconvolved into GaussianLorenzian mix functions. The deconvolved high resolution $\mathrm{C}$ 1s spectrum of film yielded three peaks at $285.4 \mathrm{eV}, 286.9 \mathrm{eV}$ and 289,4 
$\mathrm{eV}$, which are attributed to the $\mathrm{sp}^{3}$ bulk bonded carbon C-C, C-O chemical bonds and $\mathrm{C}=\mathrm{O}$ bonds (figure 3 ). The first peak at around $285.4 \mathrm{eV}$ is associated with C-C sp ${ }^{3}$ hybridization $[14,15,16]$. This can be attributed to sp3 $\mathrm{C}-\mathrm{H}$ bonding, or a diamond like structure. Peak that observed at $286.9 \mathrm{eV}$ could be C-O-C or C-O-H. Another subpeak was observed at around $289.4 \mathrm{eV}$, and is assigned to the $\mathrm{C}(\mathrm{O}) \mathrm{OH}$ or $\mathrm{HO}-\mathrm{C}-\mathrm{OH}$ bonds $[17,18]$. It should be noted that we cannot fit the data with an $\mathrm{sp}^{2}$ peak, but the presence of $\mathrm{sp}^{3}$ by itself does not confirm the DLC nature of the film, since it is possible that most sp3 bonds are terminated by hydrogen, which cannot be directly detected with the techniques used here.

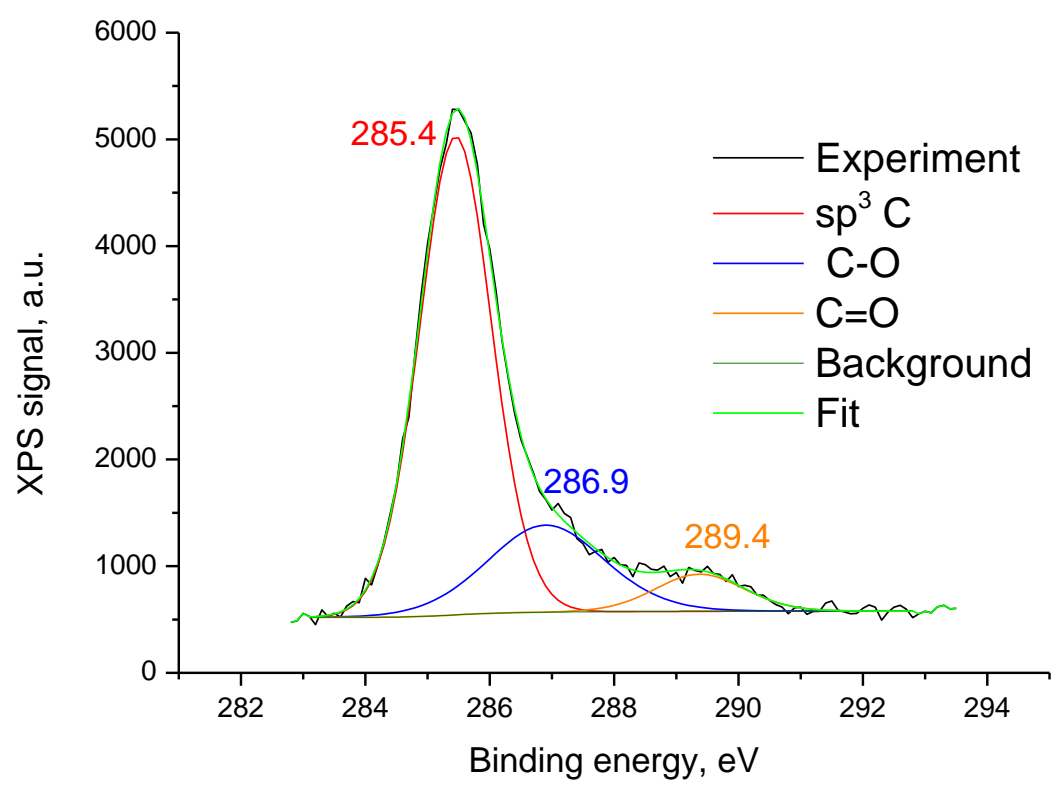

Figure 3. XPS results for carbon C1s peak. Three peaks at $285.4 \mathrm{eV}$, $286.9 \mathrm{eV}$ and $289,4 \mathrm{eV}$ are attributed to the $\mathrm{sp}^{3}$ bulk bonded carbon C-C, $\mathrm{C}-\mathrm{O}$ chemical bonds and $\mathrm{C}=\mathrm{O}$ bonds. 
Raman spectroscopy was used to characterize the molecular content of the film. Wavelengths of 264 and $514 \mathrm{~nm}$ were used to excite the Raman spectra. Additionally, Raman maps of the film were collected with a Jobin Yvon T64000 spectrometer at $514 \mathrm{~nm}$ and home-built UV Raman imaging system, based on a Ti-sapphire laser (the signal was measured on 4th harmonic) system at $264 \mathrm{~nm}$ with a resolution of 1 $\mathrm{cm}^{-1}$.

Measured Raman spectra of collector-grown carbon for ultraviolet $(264 \mathrm{~nm})$ and visible $(514 \mathrm{~nm})$ excitation wavelengths are presented in figure 4. All carbon films show similar features in their Raman spectra in the $700-2000 \mathrm{~cm}^{-1}$ region. The spectrum for $514 \mathrm{~nm}$ excitation was deconvolved into two Gaussians with maxima in the regions of the so-called D- (Disordered), and G- (Graphite) peaks, around $1360 \mathrm{~cm}^{-1}$, and $1580 \mathrm{~cm}^{-1}$, respectively. For UV excitation, a peak at $\sim 1060 \mathrm{~cm}^{-1}$ due to $\mathrm{C}-\mathrm{C} \mathrm{sp}^{3}$ vibrations, called the $\mathrm{T}$ peak, is usually observed [19-21]. The location, width, and intensity of G-, Dand T-peaks in multi-wavelength Raman spectrum provide a unique signature, which we use to characterize the carbon structure.

For UV excitation, the $G$ peak position was found to be centered at $1615 \mathrm{~cm}^{-1}$, which is blue shifted compared to typical a-C $\left(1580 \mathrm{~cm}^{-1}\right)$, or nanocrystalline graphite $\left(1600 \mathrm{~cm}^{-1}\right)$. The $\mathrm{G}$ peak position and its dispersion (see figure 4 b) is very close to that found for hydrogenated tetrahedral amorphous carbon (ta-C:H) [22], or an annealed ta-C:H film. The latter exhibits a strong D peak under 514 $\mathrm{nm}$ excitation, but shoulder-like $D$ peak under $264 \mathrm{~nm}$ excitation [23]. The carbon film grown on the collector mirror most likely consists of tetrahedral amorphous carbon (ta-C).

The UV Raman spectrum exhibited no explicit $T$ peak. The reason could be a presence of sufficient $\mathrm{sp}^{2}$ content of ta-C (oxygen bonds), 
which normally reduces the $T$ peak intensity. Instead, a D-like peak, centered at $1410 \mathrm{~cm}^{-1}$, is observed. The D-like peak is associated with the breathing mode of ring structures, however, the ring's symmetry requires a nearby defect to excite the breathing mode. Thus, the presence of the D-like peak indicates that the film has a substantial fraction of carbon atom rings, but with a significant amount of disorder [19-23]. This feature in the UV Raman spectrum, in the absence of a $\mathrm{T}$ peak, is attributed to the $\mathrm{C}-\mathrm{C}$ stretch of both hydrogenated and hydrogen free $\mathrm{sp}^{3}$ sites. From this combination of factors, we conclude that the film consists of ta-C:H film

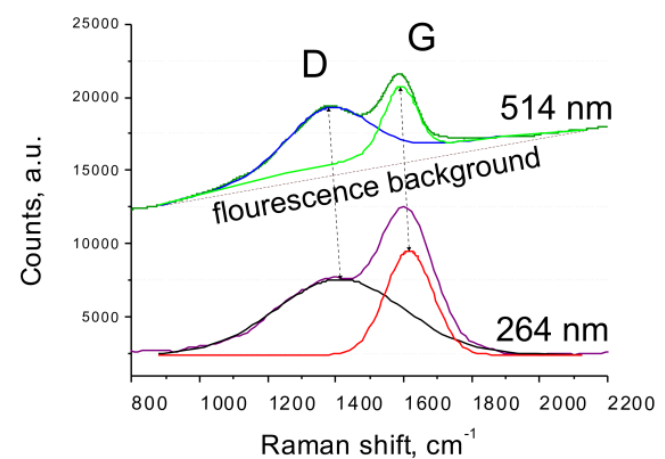

a)

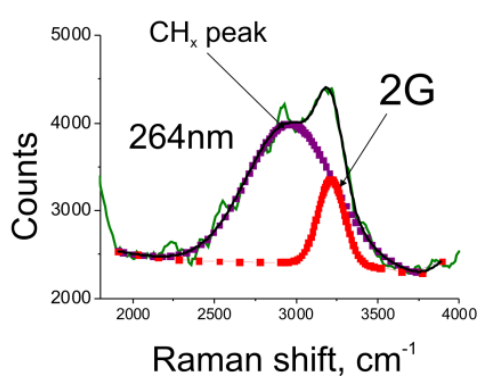

b)

Figure 4. Raman spectra of the carbon film at $514 \mathrm{~nm}$ and $264 \mathrm{~nm}$ in a range $800-2200 \mathrm{~cm}^{-1}$ (a) and in UV-Raman spectra in range 2000-4000 $\mathrm{cm}^{-1}$ (b). Peak caused by $\mathrm{sp3} \mathrm{CH}_{\mathrm{x}}$ stretching in UV-Raman (b) clearly visible.

The presence of hydrogen in the film is confirmed by Raman spectroscopy. A broad pair of peaks, shown in figure $4 \mathrm{~b}$, in the range $2500-3200 \mathrm{~cm}^{-1}$ cannot be attributed to the second order of the $D$ peak, known as the 2D' peak. Instead, we attribute this intensive 
peak to two sources: $\mathrm{sp}^{3} \mathrm{CH}_{\mathrm{x}}$ stretching vibrations at $2960 \mathrm{~cm}^{-1}$ and, the second, at $\sim 3210 \mathrm{~cm}^{-1}$, is due to the second order G- peak [24].

Since terminated $\mathrm{C}-\mathrm{H}$ bonds have a large dipole moment they can be observed directly using IR spectroscopic techniques. We used reflection absorption infrared spectroscopy (RAIRS) to observe $\mathrm{sp}^{3}$ $\mathrm{CH}_{\mathrm{x}}$ bonds. The RAIRS spectrum of the collector film in the range $2800-3100 \mathrm{~cm}^{-1}$ is shown in figure 5 . Although the absorbance of $\mathrm{sp}^{3}$ $\mathrm{CH}_{2}$ and $\mathrm{sp}^{3} \mathrm{CH}_{3}$ bonds in RAIRS is small, they are, nevertheless, clearly visible in figure 5 .

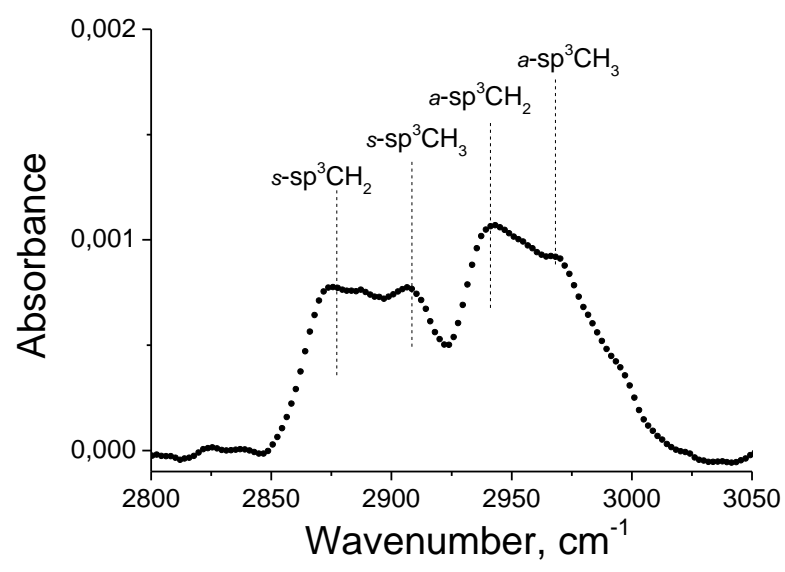

Figure 5. RAIRS absorption spectrum of the collector film in 2800 $3000 \mathrm{~cm}^{-1}$ range. Spectral resolution is $\sim 4 \mathrm{~cm}^{-1}$.

The amount of hydrogen (bound to carbon) in the film can be estimated from the ratio of the slope, $m$, of the fitted linear (fluorescent) background, and the intensity of the $G$ peak, $m / /(G)$ [24]. As shown in figure 4, the ratio $\mathrm{m} / /(\mathrm{G})$ was found to be $0.5 \mu \mathrm{m}$, which corresponds to $\sim 20$ at. \% of hydrogen. Taking into account that the XPS data does not distinguish between $\mathrm{C}-\mathrm{C}$ and $\mathrm{C}-\mathrm{H}$ bonds, we can 
conclude that the film has $\sim 20 \%$ alcohol and aldehyde groups, $\sim 20 \%$ $\mathrm{CH}_{\mathrm{x}}$ groups, while the rest of it is $\mathrm{Sp}^{3} \mathrm{C}-\mathrm{C}$ bonds.

Thus, summarizing, carbon film deposited on mirrors that are plasma-facing (e.g., the collector mirror) will not be polymer-like amorphous carbon. Instead, our results show that the film is more likely to have the properties of hydrogenated diamond-like (DLCH) carbon coatings. These coatings are known to be quite resistant to chemical and physical etching processes, especially compared to standard amorphous carbon. This result resonates well with studies of thin films (less than $20 \mathrm{~nm}$ ) reported by Nishiyama's group [25]. It was observed that the films were hydrogenated and deeper layers of EUV-induced carbon contamination demonstrate a diamond-like structure.

The plasma etching was performed to confirm that the carbon film deposited on the collector is hard and inert, like DLCH films. The collector mirrors were cleaned with $\mathrm{H}_{2}$ plasma in an inductively coupled plasma (ICP) reactor. The etch-rate of the collector-grown carbon was compared to the etch rate of magnetron sputtered carbon. The control samples consisted of a Si/Mo multilayer substrate with a $\sim 10 \mathrm{~nm}$ thick of amorphous carbon, and a silicon wafer with a $\sim 20 \mathrm{~nm}$ thick layer of amorphous carbon. Three samples were simultaneously etched in a plasma reactor, shown in figure 6.

Ion energy spectra and flux were measured using a DC\&RF floating retarded field energy analyzer (RFEA) [26-28]. The plasma density and temperature were measured using an RF compensated Langmuir probe. Through this combination of techniques, the composition, energy spectrum, and flux of ions, incident on the 
sample surface are accurately known and can be used to characterize of ion cleaning rates and mechanisms in detail.

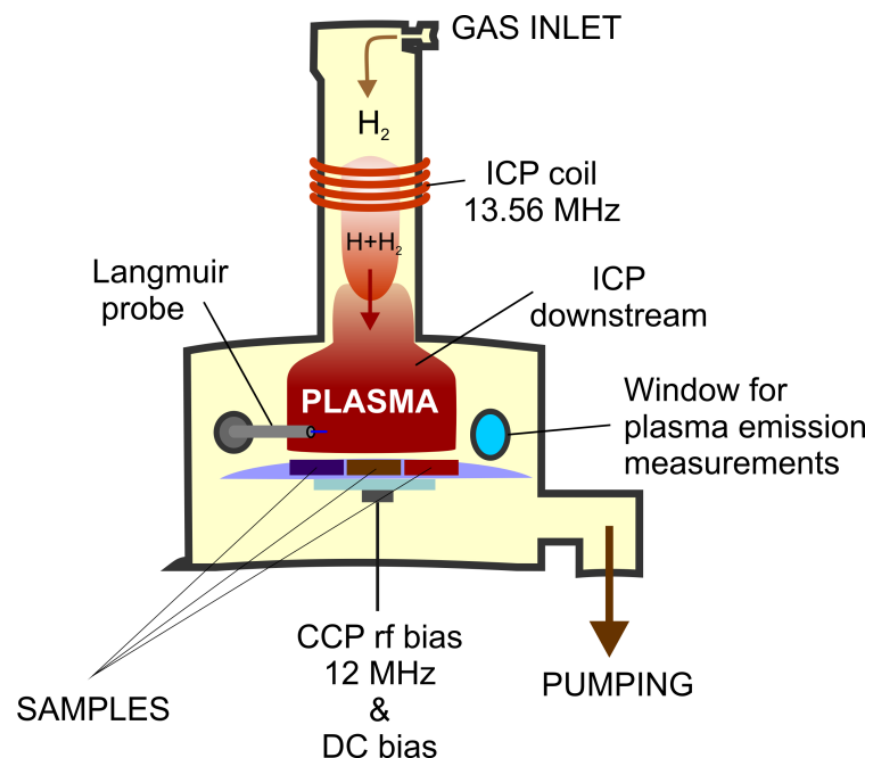

Figure 6. ICP downstream reactor. The plasma excited at $13.56 \mathrm{MHz}$ by 3-turn coil around the quartz tube with an inner diameter of $\mathbf{8 0}$ $\mathbf{m m}$. The gas pressure was maintained at $\mathbf{1 0 0}$ mTorr. Samples are placed in ICP discharge downstream at capacitively coupled plasma (CCP) rf-biased electrode. Due to a blocking capacitance and an asymmetric CCP configuration, the electrode can be DC biased and RF (12 MHz) biased.

Our plasma characterization (see Appendix 1 [29]) shows that the sample is exposed to an ion flux that is dominated by $\mathrm{H}_{3}{ }^{+}$. The composition of ions incident on the samples is presented in figure 7. The ions have a well peaked energy spectrum, which is established by an external bias field. The full width half maximum of the ion energy spectrum ranges from 4 to $45 \mathrm{eV}$, depending on the peak ion 
energy. The ion integral flux was in the range of $10^{13}-3 \cdot 10^{14}$ ions $/\left(\mathrm{cm}^{2} \cdot \mathrm{s}\right)$, depending on the applied bias.

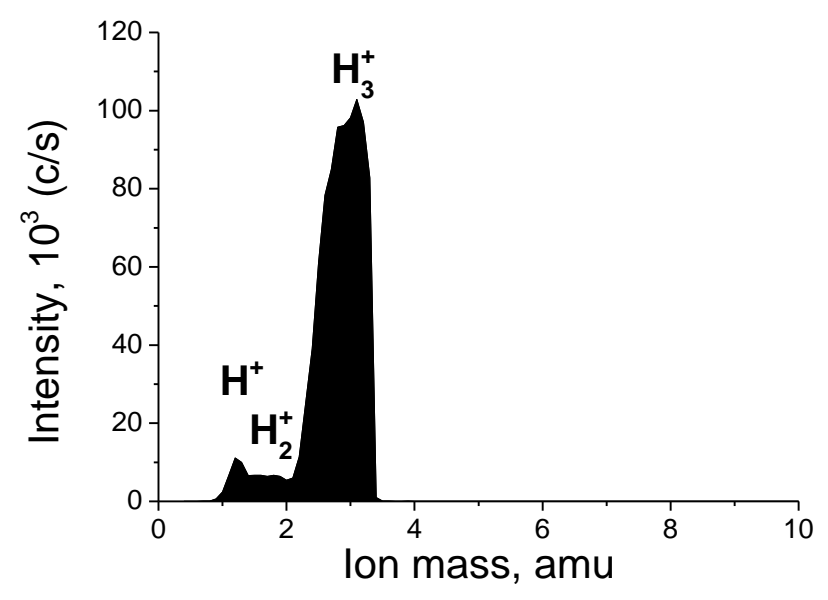

Figure 7. Measured mass-spectrum of ions incident on the sample surface from $\mathrm{H}_{2}$ plasma at an $\mathrm{H}_{2}$ pressure of $100 \mathrm{mTorr}$ and $200 \mathrm{~W}$ ICP $13.56 \mathrm{MHz}$ discharge.

The ion energy distribution functions (IEDFs) at the electrode under conditions identical to the conditions that the samples were exposed to are shown in figure 8 . In addition to ions, the surface is also exposed to atomic hydrogen. The flux of atomic hydrogen was found to be $f_{\mathrm{H}}=8 \cdot 10^{18} \mathrm{at} /\left(\mathrm{cm}^{2} \cdot \mathrm{s}\right)$.

The sample surface in the plasma is exposed to hydrogen atoms and ions. By measuring the ion dose and etch rate, we estimated the carbon atom removal probability or $\mathrm{C}$ yield per incident ion as a function of ion energy (see figure 9). In order to simplify the estimation, the density of all studied carbon films was assumed to be the same at $2 \mathrm{~g} / \mathrm{cm}^{3}$. The estimated $\mathrm{C}$ etch probability per $\mathrm{H}$ atom was about $\sim 10^{-6}$ for magnetron-deposited carbon, which is in good 
agreement with previous studies of hot filament $\mathrm{H}$ radical cleaning and plasma cleaning $[9,30]$. Therefore, it can be expected that chemical sputtering and reactive ion etching are predominant mechanisms, especially with increasing ion energy [9]. At the lowest energies, the etch rate is constant. This is most likely dominated by atomic hydrogen etching. $\mathrm{H}_{2}$ dissociation mostly occurs in the ICP 13.56 $\mathrm{MHz}$ plasma, which was kept constant during the experiment.

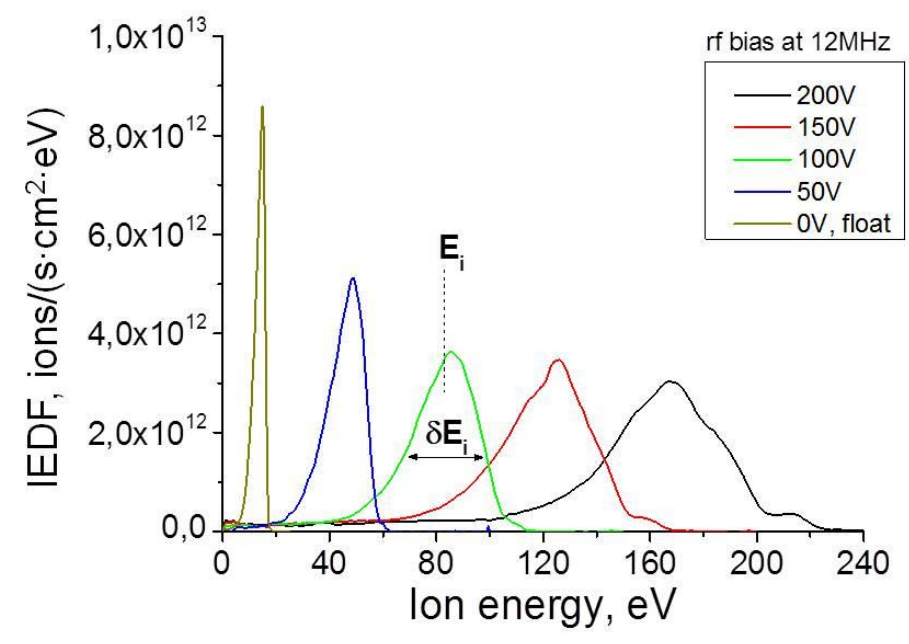

Figure 8. Ion energy distribution function at surface of $12 \mathrm{MHz}$ biased electrode (where samples were placed) at $\mathrm{H}_{2} 100 \mathrm{mTorr}$, $200 \mathrm{~W}$ ICP $13.56 \mathrm{MHz}$ discharge and different RF bias voltages.

As can be seen in figure 9, the etch probability of EUV collector grown carbon (EUC-C) is notably lower than the magnetrondeposited a-C etch probability. Furthermore, the slopes of the curves in figure 9 (etch probability vs ion energy) are different, indirectly indicating that the carbon in the two films have different binding energies. So the higher energy threshold of chemical sputtering for 
EUV-C can be explained by a harder carbon structure and, correspondingly, a higher binding energy compared to magnetron sputtered a-C films. For instance, TRIM (TRansport of Ions in Matter) software [31], used for calculating ion interaction with materials (mainly amorphous), assumes $25 \mathrm{eV}$ displacement energy for atoms in stable (hard) carbon material. As displacement energy we take the minimum kinetic energy that an atom needs to be permanently displaced from its place to a different position within a film. For amorphous or polymer-like carbons, this energy appears to be notably lower [9]. These experimental results show that the collector film is hard, and together with other data, it is most likely to be a hydrogenated DLC film.

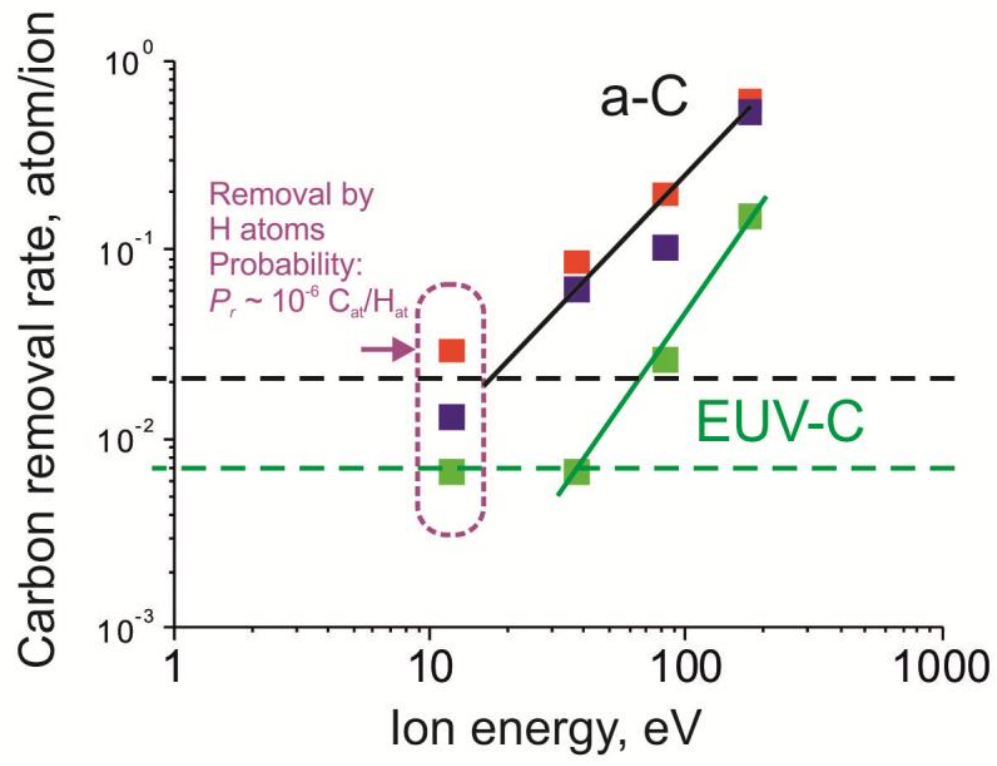

Figure 9. Carbon removal rate (atom/ion) for the different samples as function of ion energy. Dashed lines indicate approximate thresholds of chemical sputtering of amorphous magnetrondeposited and collector films. 
To a first approximation the process of collector film formation in an $\mathrm{H}_{2}$ environment can be considered to be similar to the well-known plasma enhanced chemical vapor deposition (PECVD) of hard carbon and DLC coatings. In this deposition process, plasma is used as a source of reactive species, and the substrate and film is subjected to a flux of energetic ions. The similarity between PECVD and EUVinduced deposition can be seen by considering the environmental conditions. The carbon layer is produced due to dissociation of absorbed hydrocarbons by EUV photons and secondary electrons. Dissociation occurs even in high-vacuum conditions $\left(<10^{-8}\right.$ Torr $)$ because the residence time of multi-atom hydrocarbon molecules on a surface is long. In our experiments with EUV exposure, $\mathrm{H}_{2}\left(\sim 10^{-3}\right.$ Torr) flows over the collector mirror, so the deposition proceeds in the presence of $\mathrm{H}_{2}$ and in the presence of a low density hydrogen plasma that is generated by the EUV pulse [9,32]. The plasma potential, on the other hand, is high: from a few tens of eV up to the photon energy $(91.8 \mathrm{eV})$. Therefore, the energy of incident ions will be close to the plasma potential.

Low-energy hydrogen ions preferentially etch $\mathrm{sp}^{2}$ bound species. This process has been observed in PECVD processing [10], where increasing the ion energy to a few tens of eV often leads to the formation of a hard carbon, enriched in $\mathrm{sp}^{3}$ phase compared to $\mathrm{sp}^{2}$. This occurs because $\mathrm{sp}$ and $\mathrm{sp}^{2}$ carbon reacts with hydrogen radicals and ions more readily than does $\mathrm{sp}^{3}$ bound carbon [33]. In addition to PECVD photo-dissociation of hydrogen molecules under pinch radiation should be taken into account. Simultaneous surface interaction of $\mathrm{H}$ atoms with $\mathrm{CH}_{3} \mathrm{sp}^{3}$ bonded radicals leads to increase of sticking coefficient for methyl radicals [34,35]. These processes lead to enrichment of the film structure by $\mathrm{sp}^{3}$ carbon. Moreover the space in between Z-pinch and the collector, because of the presence of EUV and high energetic Sn ions, acts as the source of 
hydrogen and hydrocarbon ions and radicals. The $92 \mathrm{eV}$ photon energy is much more than $\mathrm{C}-\mathrm{C}$ or $\mathrm{C}-\mathrm{H}$ bond energy. Radiation, hydrogen ions and heavy tin ions interaction can easily cause formation of carbon $\mathrm{C}^{+}$ions with energy more than $20 \mathrm{eV}$. This alone is already enough to produce hard $\mathrm{sp}^{3}$ rich carbon coatings. Taking into account water, absorbed on the mirror surface, the film is expected to contain also some $\mathrm{C}-\mathrm{OH}$ and $\mathrm{C}(\mathrm{O}) \mathrm{OH}$ structures because of photo dissociation and ion induced subplantation [10].

\subsection{Conclusion}

We have studied the carbon film grown from hydrocarbons in an atmosphere of $10^{-3}$ Torr hydrogen during exposure to EUV radiation from a Z-pinch tin discharge. The film contains carbon, oxygen, and hydrogen as the main elements and involves submicron tin droplets. The $\sim 150 \mathrm{~nm}$ film is transparent in the visible spectral range and chemically relatively inert in comparison with amorphous magnetron deposited carbon, as shown by its insolubility in nitric acid. Through elemental analysis, Raman spectroscopy, and plasma etching experiments, it has been shown that elements of the film are chemically bound in a mechanically and chemically tough structure, formed by $\mathrm{C}-\mathrm{C}, \mathrm{C}-\mathrm{H}, \mathrm{C}-\mathrm{OH}$ and $\mathrm{C}(\mathrm{O}) \mathrm{OH}$ chemical groups with the significant part of the carbon being in $\mathrm{sp}^{3}$ hybridization. Compared to magnetron deposited carbon, the film also has a higher resistance to chemical and physical sputtering during plasma treatment. The reduced hydrogen plasma removal rate for collector grown films can be up to an order of magnitude lower than for a-C especially for hydrogen ions energies below $100 \mathrm{eV}$. Thus the characteristics of the film obtained by both non-destructive and destructive diagnostics allow us to classify the film as diamond-like coating. One can conclude that carbon contamination of EUV optics that are close to the EUV source and in presence of EUV-induced plasma can be rather hard and are similar to hydrogenated DLC coatings. Such 
diamond-like amorphous carbon could require ten times more $\mathrm{H}_{3}{ }^{+}$ ions to remove one $\mathrm{C}$ atom during hydrogen plasma treatment. To solve the optics cleaning issue for such type of contamination it is necessary to optimize in-situ cleaning methods, such as those based on using EUV-induced plasma, as considered in [9,32]. 


\section{References}

1. Hollenshead J and Klebanoff L 2006 J. Vac. Sci. Technol. B 2464.

2. Boller K, Haelbich R P, Hogref H, Jark W, and Kunz C 1983 Nucl. Instrum. Methods Phys. Res. 208273.

3. Koster N, Mertens B, Jansen R, van de Runstraat A, Stietz F, Wedowski M, Meiling $\mathrm{H}$ et al. 2002 Microelectron. Eng. 61-62 65.

4. Lytle W M, Lofgren R E, Surla V, Neumann M J, and Ruzic D N 2010 Proc. SPIE 63676360.

5. Koida K and Niibe M 2009 Appl. Surf. Sci. 2561171.

6. Koster N, van der Donck J C J,Stortelder J K, de Jong A J, and Molkenboer F T 2012 Proc. SPIE 8322 83220R.

7. Murakami K, Oshino T, Kondo H, Chiba H, Kawabe Y, Ono T, Kandaka N, Yamazaki A, et.al. 2012 Proc. SPIE 8322, 832215.

8. Louis E, Yakshin A E, Tsarfati T, Bijkerk F 2011 Progress in Surf. Science 86255 -294.

9. Dolgov A, Lopaev D, Rachimova T, Kovalev A, Vasil'eva A, Lee C J, Krivtsun V M et al. 2014 J. Phys. D: Appl. Phys. 47065205.

10. Robertson J, 2002 Materials Science and Engineering $R 37$ 129-281

11. A Dolgov et al 2015 Plasma Sources Sci. Technol. 24035003.

12. Hirai H, Kondo K, 1991 Science 253 772-774.

13. Shirley D A 1972 Phys. Rev. B 54709.

14. Díaz J, Paolicelli G, Ferrer S, and Comin F 1996 Phys. Rev. B 54 8064.

15. Wan D and Komvopoulos K 2007 J. Phys. Chem. C 111 9891-9896.

16. Caschera D, Cossari P, Federici F, Kaciulis S, Mezzi A, Padeletti G, Trucchi D M 2011 Thin Solid Films 519 4087-4091.

17. Kozlowski C, Sherwood P M A 1984 J Chem Soc Farad T 1 Phys Chem Condensed 80(8) 2099-107.

18. Yang D, Velamakanni A, lay Bozoklu Gu, Park S, Stoller M, Piner R D, Stankovich S, Jung I, Field D A, Ventrice C A, Ruoff R S 2009 CARBON 47 145-152.

19. Piscanec S, Mauri F, Ferrari A C, Lazzeri M, and Robertson 2005 J Diamond Relat. Mater. 141078. 
20. Gilkes K W R, Prawer S, Nugent K W, Robertson J, Sands H S, Lifshitz Y, and Shi X, 2000 J. Appl. Phys. 877283.

21. Merkulov V I, Lannin J S, Munro C H, Asher S A, Veerasamy V S, and Milne W I, 1997 Phys. Rev. Lett. 784869.

22. Casiraghi C, Piazza F, Ferrari A C, Grambole D, Robertson J 2005 Diamond \& Related Materials 14 1098-1102.

23. Adamopoulos G, Gilkes K W R, Robertson J, Conway N M J, Kleinsorge B Y, Buckley, Batchelder D N 1999 Diamond and Related Materials 8 541-544.

24. Casiraghi C, Ferrari A C, and Robertson J 2005 Phys. Rev. B 72 085401.

25. Nishiyama I 2009 Update on SFET optics contamination learning, Analysis of carbon contamination, IEUVI Optics Contamination TWG, San Jose.

26. Gahan D, Dolinaj B, Hopkins M 2008 Plasma Sources Sci Technol. 17035026.

27. Hopwood J 1993 Applied Physics Letters 62940.

28. Mizutani N, Hayashi T 2001 Journal of Vacuum Science \& Technology A 191298.

29. Appendix 1

30. Braginsky $O \vee$, Kovalev A S, Lopaev D V, Malykhin E $M$, Rakhimova T V, Rakhimov A T, Vasilieva A N, Zyryanov S M, Koshelev K N, Krivtsun V M, M van Kaampen and Glushkov D 2012 J App. Phys. 111093304.

31. http://www.srim.org/

32. van der Velden $M H$ L, Brok W J M, van der Mullen J J A M, Goedheer W J, and Banine V 2006 Phys. Rev. E 73.3036406.

33. Villalpando I, John P, Porro S, Wilson J I B 2011 Diamond and Related Materials 20 711-716.

34. von Keudell A, Schwarz-Selinger T, Meier M and Jacob W 2000 Appl. Phys. Lett. 76676.

35. von Keudell A, Meier M, Hopf C 2002 Diamond and Related Materials 11 969-975. 


\section{Plasma-assisted oxide removal from ruthenium coated EUV optics}

\section{Abstract}

An experimental study of oxide reduction at the surface of ruthenium layers on top of multilayer mirrors is presented. Oxidation and reduction processes were observed under conditions close to those relevant for extreme ultraviolet lithography. The oxidized ruthenium surface was exposed to a low-temperature hydrogen plasma, similar to the plasma induced by extreme ultraviolet radiation. The experiments show that hydrogen ions are the main reducing agent. Furthermore, the addition of hydrogen radicals increases reduction rate beyond that expected from simple flux calculations. We show that low-temperature hydrogen plasmas can be effective for reducing oxidized top surfaces. Our proof-ofconcept experiments show that an in situ, EUV generated plasma cleaning technology is feasible.

\section{Introduction}

Photolithographic technology, employing extreme ultraviolet (13.5 $\mathrm{nm}$ wavelength) radiation, has seen significant progress recently [1]. Multilayer mirror (MLM) optics, comprised of $~ 50-60$ bi-layers of Mo:Si, each layer being $6.7 \mathrm{~nm}$ thick, are used in this spectral range. To maximise the lifetime of such optics, oxidation of the top protective coating under the intensive EUV radiation [2] should be prevented. Oxidation can be controlled by coating the mirrors with few $n$ m of ruthenium.

Water molecules, which are physically adsorbed on the ruthenium surface, absorb EUV photons. This may either partially or fully 
dissociate the water, leading to the formation of atomic oxygen. Atomic oxygen generation allows oxidation to proceed during EUV radiation. It is note worthy that electron irradiation at similar energies ( 100 eV) does not result in such intense Ru oxidation [3].

To control oxidation, a technology that efficiently and precisely cleans MLM surfaces must be available. The contamination and cleaning of EUV optics has been subject to intense research recently [4-8]. Atomic hydrogen is currently applied to remove different types of contaminations $[5,9,10]$. However, atomic hydrogen is not a very effective agent for ruthenium oxide reduction [10]. In the case of exposure to high-energy photons, photoionisation prevents the formation of a high-density flux of $\mathrm{H}$ atoms towards the irradiated surface. It imposes considerable limitations on the cleaning speed and selectivity. On the other hand, plasma sources are known to efficiently reduce oxide layers from many surfaces [11-13]. However, these plasmas are typically high density, high temperature plasmas and, as such, are incompatible with EUV mirrors [14,15].

An alternative is to use the plasma generated by ionization due to EUV radiation. The photon energy of EUV radiation is $92 \mathrm{eV}$, sufficient to ionize hydrogen. Thus, direct photo-ionization of the background gas, along with secondary ionization due to electrons that are emitted from MLMs, creates a short-lived plasma above the mirror surface, which may clean these surfaces in-line [15].

Earlier works [16] have proven that carbon can be efficiently etched from multilayer mirrors in an EUV-induced hydrogen plasma. This suggests that in-line cleaning could also work for surface oxides as well. 
In the absence of EUV the rates of Ru oxidation and reduction are governed by the equations:

$$
\begin{aligned}
& d\left[\mathrm{~N}_{\mathrm{RuOx}}\right] / d t=\mathrm{k}_{1}\left[\mathrm{~N}_{\mathrm{H} 2 \mathrm{O}}\right]\left[\mathrm{N}_{\mathrm{Ru}}\right]-\mathrm{k}_{2}\left[\mathrm{~N}_{\mathrm{H} 2}\right]\left[\mathrm{N}_{\mathrm{RuOx}}\right] \\
& \mathrm{d}\left[\mathrm{N}_{\mathrm{Ru}}\right] / d t=\mathrm{k}_{2}\left[\mathrm{~N}_{\mathrm{H} 2}\right]\left[\mathrm{N}_{\text {RuOx }}\right]-\mathrm{k}_{1}\left[\mathrm{~N}_{\mathrm{H} 2 \mathrm{O}}\right]\left[\mathrm{N}_{\mathrm{Ru}}\right]
\end{aligned}
$$

where $\mathrm{N}_{\mathrm{Ru}}, \mathrm{N}_{\mathrm{RuOx}}, \mathrm{N}_{\mathrm{H} 2}, \mathrm{~N}_{\mathrm{H} 2 \mathrm{O}}$ numbers are surface densities for $\mathrm{Ru}$, RuOx, $\mathrm{H}_{2}$ and $\mathrm{H}_{2} \mathrm{O}$ (the latter two being the main reducing and oxidising agents). $k_{1}$ and $k_{2}$ are effective rate coefficients of $\mathrm{Ru}$ oxidation and restoration from RuOx by hydrogen. Furthermore, the ratios of the number densities depends on the partial pressures of molecular hydrogen and water in a gas phase and desorption temperatures of these species. Hydrogen desorption starts from clean ruthenium $\mathrm{Ru}(0001)$ at $400 \mathrm{~K}$ and for oxidized surface $\mathrm{RuO}_{2}(110)$ at $300 \mathrm{~K}$, while water desorption starts from ruthenium $\mathrm{Ru}(0001)$ at $150 \mathrm{~K}$ and for oxidized surface $\mathrm{RuO}_{2}(110)$ at $200 \mathrm{~K}[5,17]$. Since the partial pressure of at least one of these chemical agents $\left(\mathrm{H}_{2}, \mathrm{H}_{2} \mathrm{O}\right)$ can be controlled in most vacuum systems, the surface coverage is often, to first order, under experimental control.

However, although the number densities of the background gases are under control, the gases and surface adsorbed species are often partially dissociated or ionized. For instance, on a Ru surface, a fraction of the adsorbed water partially dissociates. Absorption of EUV leads to the formation of oxygen radicals, vastly increasing the rate of oxide formation. On the other hand, hydrogen also dissociates on $\mathrm{Ru}$, forming more active radical species, while the presence of EUV leads to a large radical and ion flux incident on the surface, changing the rate at which reduction occurs. Assuming linearity (e.g., that all rates are independent of each other), these processes can be taken into account in composite rates, $k_{1}$ and $k_{2}$. 
The rates $k_{1}$ and $k_{2}$ must be known, and the major contributors to the composite rate understood in order to control the balance between oxidation and reduction.

Although oxidation of ruthenium surfaces has been described by a model, studies of reduction and oxidation are limited [18]. Specifically, the reduction of the surface, and the balance between oxidation and reduction have not been studied in detail. Furthermore, the balance between the oxidation and reduction under EUV radiation, and, consequently, in the presence of ions and radical species has not been investigated.

The aim of this chapter is to present an analysis of the main mechanisms for oxide reduction. Thus, the potential for balancing oxidation and reduction through the use of EUV-generated active species (ions and radicals) can be evaluated. Specifically, the possibility for in-line cleaning, via the low-pressure hydrogen plasma that is induced by EUV radiation, is considered.

We present new data on the oxidation and reduction of the top ruthenium surface of MLMs in the presence of EUV radiation. A low density, inductively coupled, plasma and a surface wave discharge plasma were used to determine the absolute etch rates due to hydrogen ions, radicals, and the combination of the two. We conclude that, under EUV lithography relevant conditions, efficient oxide removalis possible using in-line plasma cleaning.

\subsection{Oxidized ruthenium samples}

EUV-induced ruthenium oxidation/reduction processes were studied under a variety of exposure conditions and partial pressures of 
hydrogen and water. We chose experimental conditions that are as close as possible to those relevant to EUV lithography. Hence, for these experiments, MLM samples, consisting of Mo/Si multilayers $(25 \times 25 \mathrm{~mm})$, terminated with $<3 \mathrm{~nm}$ of ruthenium, were used.

To study the influence of ion energy and flux, compared to radical flux, oxidized ruthenium layers were reduced in a plasma reactor that produces plasmas that are very similar to those produced by EUV radiation. The samples consisted of plasma oxidized ruthenium films, which are structurally and chemically similar to ruthenium oxide films that grow under EUV illumination [5]. This model system is more straightforward to analyze than a regular MLM. Furthermore, by using a relatively thick Ru layer, the samples could also be used as electrodes to measure the ion flux.

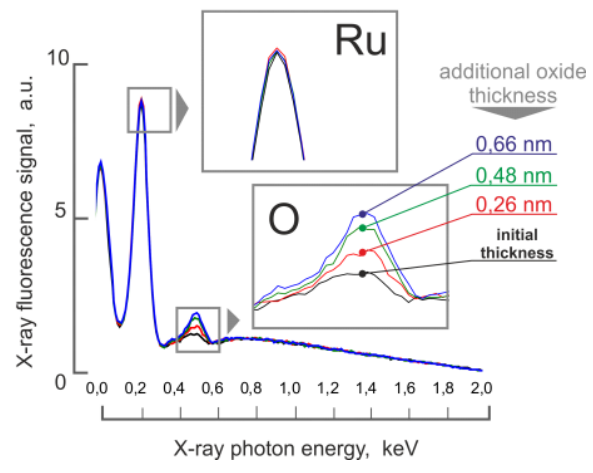

a)

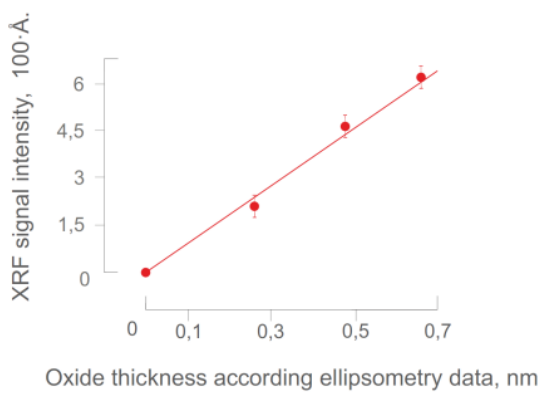

b)

Figure 1. X-ray fluorescence analysis of the oxidized $20 \mathrm{~nm}$ thick Ru film on $\mathrm{Si}$. The presence of an oxygen peak (525 eV) shows that the ruthenium surface is always oxidized in the case of ex-situ measurements. For our experiments, the initial oxide thickness is less than one monolayer (a) and calibration curve for ellipsometry and XRF is linear (b). 
The ruthenium oxide layer thicknesses were measured using ellipsometry, Energy Dispersive Spectroscopy (EDS), and X-ray fluorescence analysis (XRF) (see Figure $1 a, b$ ). Figure $1 b$ shows $a$ typical calibration curve for ellipsometry and XRF. The symbols show intensity of the EDS and the model-predicted ruthenium oxide thickness, based on measured ellipsometry data. The model uses a modified set of optical constants obtained from Palik [19]. The oxide layers were found to have thicknesses of $0.26 \mathrm{~nm}, 0.48 \mathrm{~nm}$ and 0.66 $\mathrm{nm}$. These thicknesses correspond to less than 1 monolayer $(\mathrm{ml})$ of oxide, more than $1 \mathrm{ml}$ and less than $2 \mathrm{ml}$ of oxide and more than 2 $\mathrm{ml}$ of oxide respectively, assuming $\mathrm{RuO}_{2}(110)$ oxide structure. Three thicknesses of oxide film were chosen so that the influence of oxide depth on oxide reduction could be studied.

A linear regression of the data results in a measurement accuracy of $\pm 0.05 \mathrm{~nm}$ for ellipsometry. For the plasma-assisted reduction experiments, only ex-situ EDS measurements were performed.

\subsection{Evolution of oxidation and reduction of ruthenium in water/hydrogen medium.}

EUV-induced oxidation and reduction was studied in an EUV exposure tool. A schematic representation of this setup is given in figure 2. The tool consists of two main parts: the EUV source and the exposure chamber. The exposure chamber is separated from the radiation source by a spectral-purity filter (SPF) which ensures that the sample is only exposed to EUV light. The SPF is a free-standing SiZr filter with $50 \mathrm{~nm}$ thickness and 78\% in-band transmission. The EUV beam (FWHM $~ 4 \mathrm{~mm}$ ) was focused to a $0.5 \mathrm{~cm}$ diameter spot with an average power density of $5.7 \mathrm{~mW} / \mathrm{mm}^{2}$ at $600 \mathrm{~Hz}$ during 35 million pulses. 
Within the exposure chamber, samples are irradiated at the focus of the EUV beam under varying background gas pressures and sample temperatures. The base pressure of the system after bake-out is $4 \cdot 10^{-8} \mathrm{mBar}$. Water and hydrogen are introduced to the exposure chamber via two separate valves. For in-situ oxidation/reduction studies, the natural oxide layer due to exposure to atmosphere was first removed by hydrogen radical etching within the load lock chamber.

While the sample is being irradiated, an imaging ellipsometer allows for in-situ monitoring of changes to the sample surface (e.g. growth or reduction of surface oxide) with sub-nm sensitivity; with this technique the oxidation and reduction of the surface can be spatially and temporally resolved.

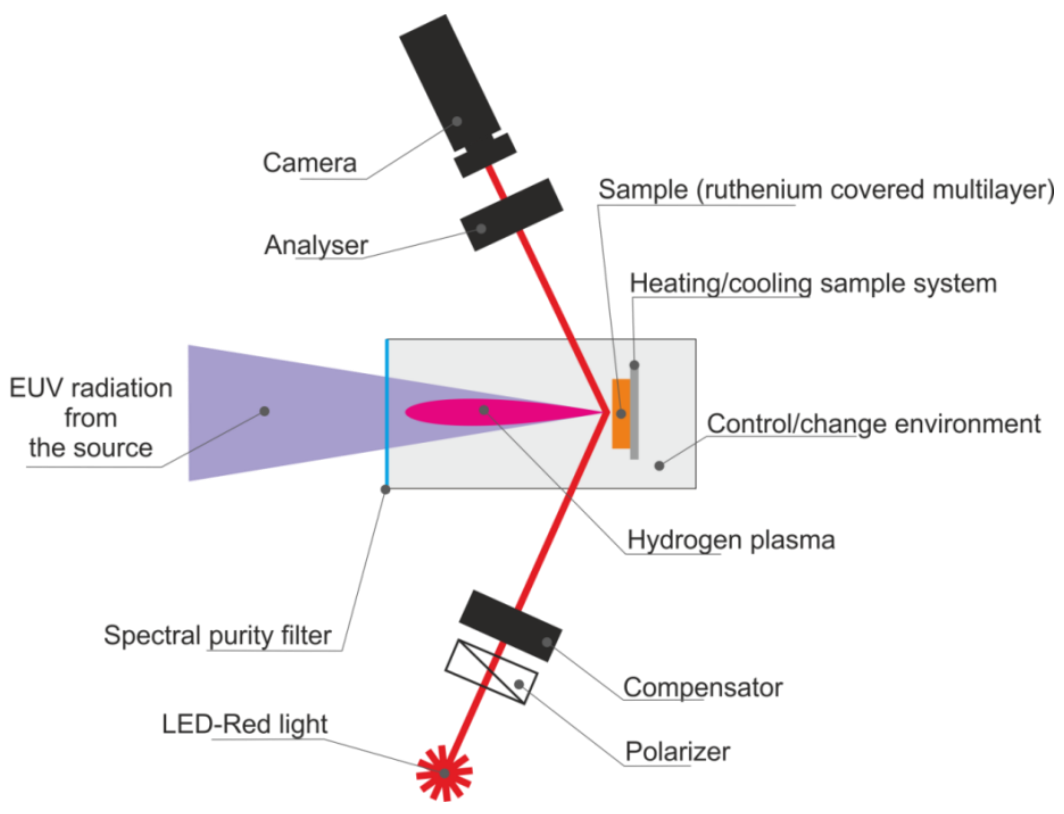

Figure 2- EUV exposure tool. 
The imaging ellipsometer was developed in-house. It is a rotating compensator ellipsometer in a polarizer-compensator-sampleanalyzer configuration. It uses a $\sim 630 \mathrm{~nm}$ LED light source that is collimated to a $\sim 2.5 \mathrm{~cm}$ diameter beam that is incident at a 70 degree angle on the sample. The reflected light is detected by a $2 / 3^{\prime \prime}$ CCD camera with a $75 \mathrm{~mm}$ focal length lens. The timing of the camera trigger is synchronized to the rotation of the compensator, allowing one to determine $\Psi$ (the amplitude ratio upon reflection) and $\Delta$ (phase shift) for each pixel.

Samples were held at a fixed temperature using a closed cycle system (Phoenix II, Thermoscientific). The minimum sample temperature is limited by the thermal contact between the cooling circuit and the MLM. The actual temperature is measured by a small (2x2 mm) PT1000 resistive temperature sensor $\left(3.8 \Omega /{ }^{\circ} \mathrm{C}\right)$ that is clamped on the metallic frame that holds the mirror.

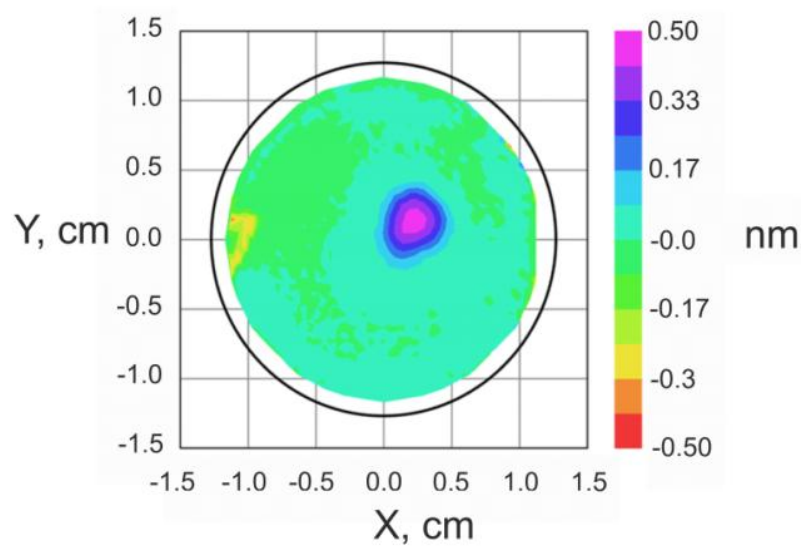

Figure 3. Ruthenium oxide thickness map. The sample was oxidized at $2.10^{-5} \mathrm{mBar}$ of partial water pressure and $4 \cdot 10^{-8} \mathrm{mBar}$ of partial hydrogen pressure. Surface temperature was $8^{\circ} \mathrm{C}$. The EUV power was $5.7 \mathrm{~mW} / \mathrm{mm}^{2}$ at $600 \mathrm{~Hz}$ and the total number of EUV pulses was $35 \cdot 10^{6}$. 
An example of the oxide thickness determination, as extracted from the optical constants is presented in figure 3 .

In order to illustrate control for oxidation and reduction of the MLM protective layer, we placed the samples under the EUV radiation and monitored the oxide thickness under different $\mathrm{H}_{2} \mathrm{O}: \mathrm{H}_{2}$ ratios using ellipsometry. Figure 4 shows the evolution of $\mathrm{Ru}$ oxidation and reduction as the partial pressures of water and hydrogen are varied. The $\mathrm{RuO}_{\mathrm{x}}$ thickness and phase shift ( $\Delta$-signal) are plotted as a function of exposure time. The experiment began with a sample that was oxidized to a depth of $0.15 \mathrm{~nm}$. First, the ability to obtain a balance between oxidation and reduction is demonstrated by exposing the sample to a mixture of water and hydrogen (partial pressures of $\mathrm{P}_{\mathrm{H} 20}=2 \cdot 10^{-5} \mathrm{mBar}$ and $\mathrm{P}_{\mathrm{H} 2}=5 \cdot 10^{-2} \mathrm{mBar}$ at a temperature of $4^{\circ} \mathrm{C}$ ). Here, to within our measurement accuracy, an equilibrium is observed: oxidation is balanced by oxide reduction.

Once the partial pressure of water is reduced to background levels $\left(\sim 10^{-8} \mathrm{mBar}\right)$ (30 min- $64 \mathrm{~min}$, figure 4$)$, reduction dominates. In this case, $\Delta$ increases, indicating the reduction of the oxide thickness from 0.15 to $0.08 \mathrm{~nm}$. We also demonstrate that this can be reversed (64 min -140 min, figure 4) by reducing the hydrogen partial pressure to background levels and returning the water partial pressure to $\mathrm{P}_{\mathrm{H} 20}=2 \cdot 10^{-5} \mathrm{mBar}$. Under these conditions, as expected, $\Delta$ reduces, indicating oxidation of the Ru.

The details of the mechanisms underlying the balance between oxidation and reduction requires a separate study and is not the subject of the current chapter. Here we focus on an empirical model that describes the balance. 
The rate of oxide reduction and growth, obtained for $\mathrm{H}_{2}$ only and $\mathrm{H}_{2} \mathrm{O}$ only are very close in absolute value, suggesting a first-order approximation:

$$
d z / d t=a_{r}\left(P_{H 2 O}, P_{H 2}\right)+a_{o x}\left(P_{H 2}, P_{H 2}\right)
$$

Where $z$ is oxide thickness and $\alpha$ is the slope of $\Delta$ (corresponds to oxide thickness) vs time plot. Reduction, $\boldsymbol{a}_{\mathbf{r}}$, is considered to be positive $\left(0,018 \pm 0,01\right.$ from figurev4) and $\boldsymbol{a}_{\mathbf{o x}}$, oxidation, is negative ($0,022 \pm 0,01$ from figure 4).

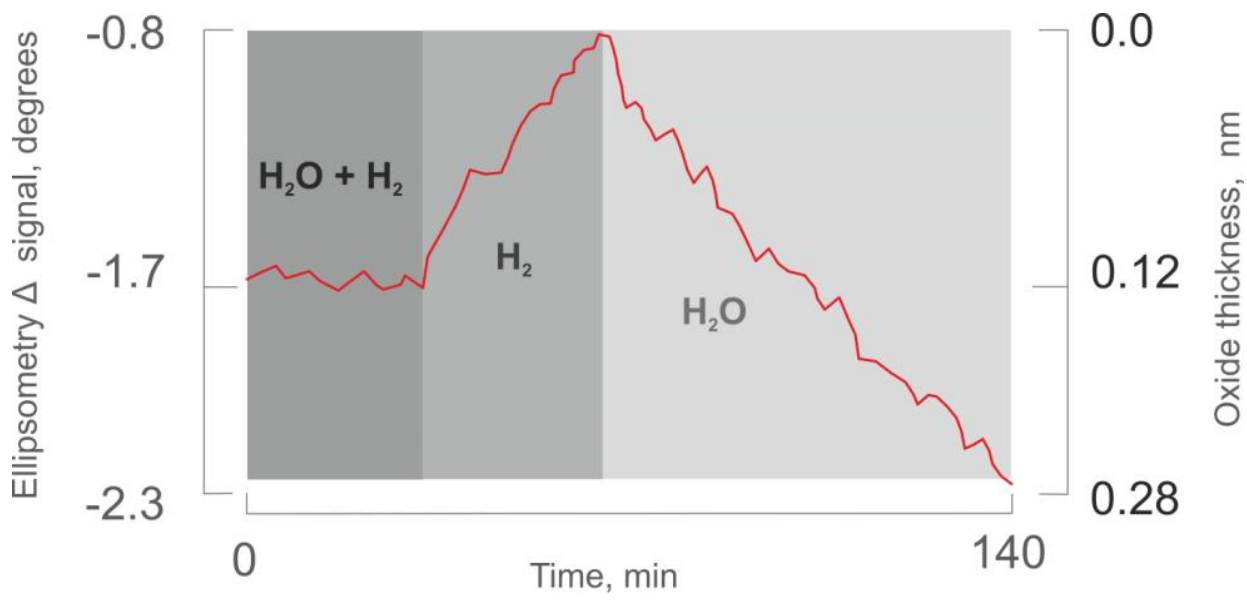

Figure 4. In-situ oxide thickness as a function of time when varying the partial pressures of water and hydrogen. The change in $\Delta$, which is approximately linear with oxide thickness in this range, is used as an approximation for oxide thickness. The sample, oxidized to a thickness of $0.12 \mathrm{~nm}$, is first exposed to a balanced water $\left(P_{\mathrm{H} 20}=2 \cdot 10^{-5} \mathrm{mBar}\right)$ and hydrogen ( $\left.P_{\mathrm{H} 2}=5.10^{-2} \mathrm{mBar}\right)$ environment. Then a hydrogen dominant environment $\left(\mathrm{P}_{\mathrm{H} 2}=5.10^{-2} \mathrm{mBar}, \mathrm{P}_{\mathrm{H} 20}<2.10^{-8} \mathrm{mBar}\right)$ and finally to a water dominant environment $\left(P_{\mathrm{H} 2 \mathrm{O}}=2 \cdot 10^{-5} \mathrm{mBar}, \mathrm{P}_{\mathrm{H} 2}<5 \cdot 10^{-8} \mathrm{mBar}\right)$. For all measurements, the sample temperature was maintained at $4^{\circ} \mathrm{C}$. 
The validity of equation (1) was checked directly by comparing measured to predicted oxide thickness growth rates at varying partial pressures of hydrogen and water. Figure 5 shows the measured oxidation/reduction rates for water/hydrogen presence only and experimental data for the case of presence of both water and hydrogen. The total $\mathrm{d} z / \mathrm{d} t$ rate from (1) for the $2 \cdot 10^{-5} \mathrm{mBar} \mathrm{H}_{2} \mathrm{O}$ environment pressure at different partial pressure of hydrogen was calculated using data for pure hydrogen $\left(a_{r}\right)$ and pure water $\left(a_{o x}\right)$ environments. The experimental value for the total rate is in good agreement with that calculated from the first order approximation.

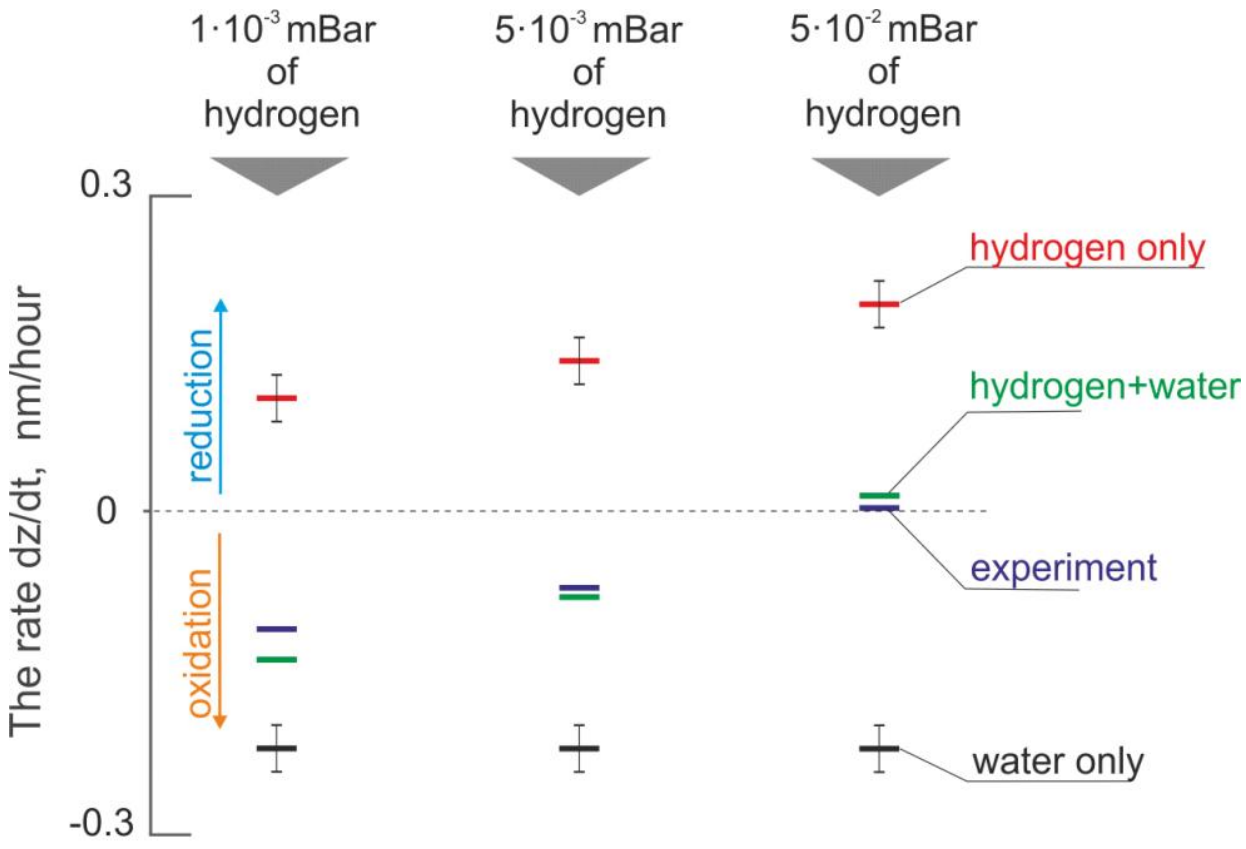

Oxidation/Reduction rates for the $2 \cdot 10^{-5} \mathrm{mbar}$ water pressure

Figure 5. Observed oxidation/reduction rate (purple), predicted oxidation/reduction rate (blue) for different partial pressures of hydrogen and a fixed partial pressure of water. Absolute oxidation (black) for constant $\mathrm{H}_{2} \mathrm{O}$ pressure only and reduction (red) rates for different partial pressures of $\mathrm{H}_{2}$ only. 
It can also be seen (see figure 4) that with increasing hydrogen pressure, oxygen removal is sufficiently fast to avoid Ru oxidation.

In summary, we observed that, under EUV radiation, the oxidation and reduction rates are only dependent on their respective feedgases rates. It is worth noting that the experiments were carried out at relatively low temperatures $\left(4{ }^{\circ} \mathrm{C}\right)$ in order to enhance the oxidation process. At higher temperature, oxidation at $\mathrm{P}_{\mathrm{H}_{2} \mathrm{O}}=2 \cdot 10^{-5}$ mBar is not observed. The physical mechanism of this process should be determined, however, we assume that the main factors could be surface activation of ruthenium or carbon growth. With increasing hydrogen partial pressure, hydrogen ions will be formed into the exposure chamber. The high energy EUV-photons ionize background gaseous hydrogen as well as the hydrogen molecules adsorbed on the surface. Secondary electrons significantly influence this process. One can assume that the low-pressure EUV-induced hydrogen plasma that formed above the sample is primarily responsible for ruthenium reduction.

\subsection{Plasma-assisted ruthenium reduction}

To study plasma assisted $\mathrm{Ru}$ oxide reduction mechanisms, we performed experiments with a hydrogen discharge plasma in conditions that closely reproduce those of an EUV-induced plasma.

Samples with varying thicknesses of ruthenium oxide were placed in a surface wave discharge plasma reactor, shown schematically in Figure 6. The samples were placed along a long quartz tube with an inner diameter of $55 \mathrm{~mm}$, where a low-pressure low-density $\mathrm{H}_{2}$ plasma is generated $[14,15,16]$. The plasma is generated in pure hydrogen, at a pressure of 100 mTorr (13.3 Pa), using an electrodeless surface wave discharge (SWD) with a power of $\sim 10-12 \mathrm{~W}$, and 
oscillating at a frequency of $40 \mathrm{MHz}$. Due to a surface electromagnetic wave, a quasi-neutral plasma column is produced in the tube beyond the SWD antenna. The electron temperature is nearly constant along the plasma column, while the plasma density gradually drops off as the distance from the antenna increases [16]. These characteristics mean that the plasma density and the corresponding ion flux vary by up to two orders of magnitude, depending on the sample position, while the ion energy spectrum remains the same for all samples. The ion flux incident on the sample surfaces was, for each sample position, derived from the analysis of IV curves for flat Langmuir probes [16]. To vary the energy of the incident ion flux, the sample holders were negatively biased. Owing to the fast ion conversion reaction: $\mathrm{H}_{2}^{+}+\mathrm{H}_{2} \rightarrow \mathrm{H}_{3}{ }^{+}+\mathrm{H}$ $\left(k=1.5 \cdot 10^{-9} \mathrm{~cm}^{3} / \mathrm{s}\right)$, the dominate ion is $\mathrm{H}_{3}{ }^{+}[14-16,21]$.

One major difference between the generated plasma described above and the EUV induced plasma is that the latter consists of a mixture of hydrogen radicals and ions, while the radical component of the former is negligible. To generate radicals, a flow of molecular hydrogen passes through the smaller-diameter quartz tube, where it is dissociated by an ICP $(13.56 \mathrm{MHz}, 200 \mathrm{~W})$ discharge. Under the given discharge conditions, up to $\sim 40 \%$ of molecular hydrogen is dissociated. However, $\mathrm{H}$ atom recombination, mainly at the surfaces of the metallic flanges that couple the quartz tubes [22], is rapid, so only about $1-2 \%$ of atomic hydrogen reaches the sample holders. Despite rapid recombination, the atomic hydrogen contribution from the $13.56 \mathrm{MHz}$ discharge is an order of magnitude more than that from the SWD $40 \mathrm{MHz}$ discharge. The density of hydrogen radicals over the sample positions was measured using actinometry. To calibrate the actinometry signal, $10 \%$ of argon was added to the hydrogen flow and the difference in the ratio of the emission spectrum lines was used to estimate the density of dissociated 
hydrogen over the samples [21,22]. The ion and atomic hydrogen fluxes, incident on the sample surfaces, were calculated from the measured ion and atom densities by using "flat-probe" approach and ideal gas relations, respectively.

Thus, the combination of a low-power SWD plasma with an ICP source for $\mathrm{H}$ atoms production allows for the effects of low-density hydrogen plasma, atomic hydrogen, and the combination of these two, to be studied in a controlled manner.

The rate of O removal from the oxidized Ru surface was obtained for three cases: samples were treated by $\mathrm{H}$ atoms only; $\mathrm{H}_{3}+$ ions only; and $\mathrm{H}_{3}+$ ions and $\mathrm{H}$ atoms at the same time.

ICP discharge hydrogen atoms source

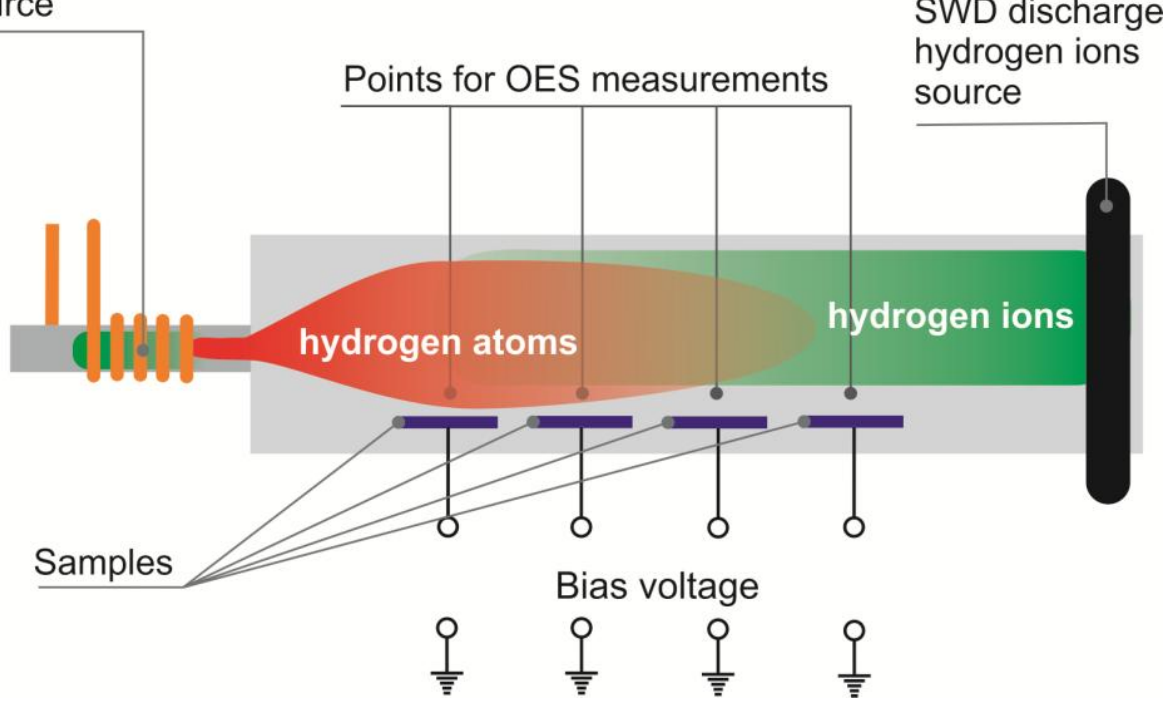

Figure 6. The SWD plasma source (right hand side) generates ions with a constant energy spectrum but decreasing density. The ICP plasma reactor (left hand side) was used to generate $\mathrm{H}$ atoms. As a result, the different sample positions are subjected to different ratios of ions to radicals. 
The best reduction effect was observed for the case of joint exposure to hydrogen atoms and ions. Ions are more effective than atoms, and even a low ion flux was observed to increase the removal rate noticeably. The oxygen removal rate by $\mathrm{H}$ atoms only is relatively low (see figures 6 and 7). In order to describe the ruthenium reduction phenomenologically, a linear approximation has been used, as it was for C-cleaning in [16].

We present the following rate equation for ruthenium reduction:

$$
R=\beta\left(E_{a c t}, T_{S} . .\right) \cdot F_{H}+\xi\left(E_{i}, T_{S}, . .\right) \cdot F_{i}
$$

Where $F_{i}$ and $F_{H}$ are the hydrogen ion and atom fluxes, respectively; $\beta\left(E_{a c t} T_{s}\right)$ is a coefficient that can be defined as the efficiency with which $\mathrm{H}$ atoms remove $\mathrm{O}$ atoms from the surface. $E_{\text {act }}$ is the activation energy of the surface reaction (typically $\sim 0,5 \mathrm{eV}$ ) and $T_{s}$ is the surface temperature. The parameter $\xi\left(E_{i}, T_{s}\right)$ is a dimensionless coefficient for ions, defined as the efficiency with which $\mathrm{H}_{3}{ }^{+}$ions removes $O$ atoms from the surface. $\xi\left(E_{i j}, T_{s}\right)$ and $\beta\left(E_{a c t} T_{s}\right)$ depend on many parameters, including surface temperature and, in the case of $\xi$, the ion energy, $E_{i}$.

Of course, such a description does not directly take into account the transport of oxygen from below the surface, the mechanism by which surface vacancies are generated etc. However, this model is sufficient to predict in-situ reduction of Ru under conditions that are close to those applied in EUVL. In EUVL, the goal is to keep the oxide layer as thin as possible, so reduction reactions of ultrathin layers are dominated by surface reactions, rather than diffusion to the surface. Furthermore, all the governing physics and chemistrysurface vacancies, for example-are absorbed into the empirically determined rates. 
Figure 7 shows the dependence between the oxygen removal rate and the flux of hydrogen atoms. As expected, the oxygen removal rate increases with increasing hydrogen atom flux. Furthermore, in the range of fluxes investigated here, the oxygen removal rate is approximately linear. The efficiency of a single hydrogen atom removing an oxygen atom is estimated to be $\beta \approx(1.4 \pm 0.3) \cdot 10^{-6}$ from the slope of a linear fit to the experimental data from figure 6 .

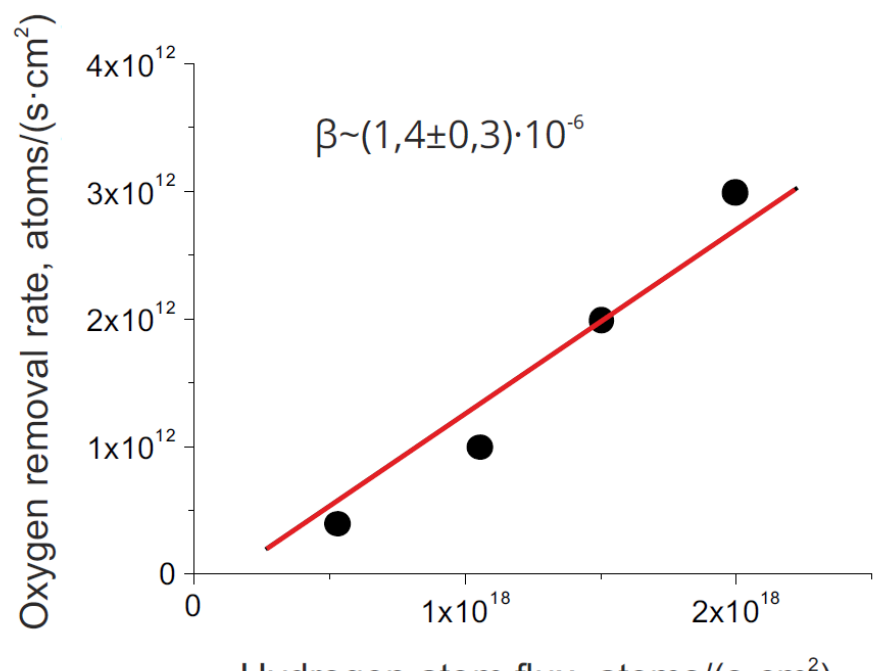

Hydrogen atom flux, atoms $/\left(\mathrm{s} \cdot \mathrm{cm}^{2}\right)$

Figure 7. The dependence of ruthenium reduction rates on atomic hydrogen flux. The per-H-atom efficiency of removing an oxygen atom is estimated from a linear fit to the data.

Figure 8 shows the oxygen removal rate for the case of ions, and for the case of a typical mixture of radicals and ions. The slope of the non-saturated part of the curve is estimated from a linear fit to the data. For the grounded samples, the efficiency, $\xi$, was found to be $\sim 0.13$, and somewhat larger ( $\sim 0.2)$ for a mixture of radicals and ions. Clearly, ions are more effective than hydrogen radicals alone. This 
can also be seen by comparing the absolute fluxes of ions and radicals. The ion flux is four orders of magnitude less than for radicals (see figure 6), while the reduction rate for ions is still an order of magnitude more than for radicals alone.

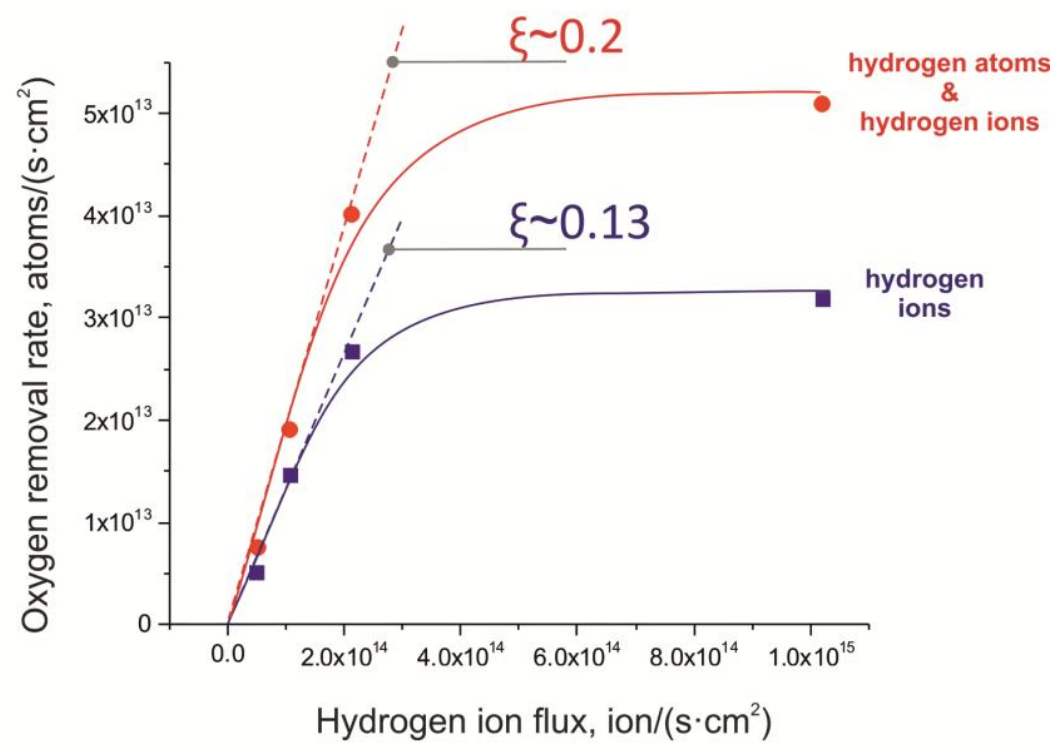

Figure 8. Effectiveness of oxygen removal from grounded samples. The ruthenium reduction rate was measured for the following cases: surface wave discharge is ON for ion exposure (ions only); and surface wave and ICP discharges are switched onto obtain a mixture of atoms and ions. The initial slopes (slopes of dashed lines) were used to define the per-ion 0 removal rates.

To compare the effectiveness of reduction under various conditions, we estimate the average number of oxygen atoms removed by a single hydrogen ion, which we refer to as reduction efficiency, $\xi$. The dependence of $\xi$ on ion energy, shown in figure 9, was estimated by measuring reduction rates on biased samples. It can be seen that $\xi$ rises slowly with increasing ion energy at energies below $\sim 40 \mathrm{eV}$. But 
for energies greater than $40 \mathrm{eV}, \xi$ begins to increase sharply and reaches a value of more than 2 for ion energies $>100 \mathrm{eV}$.

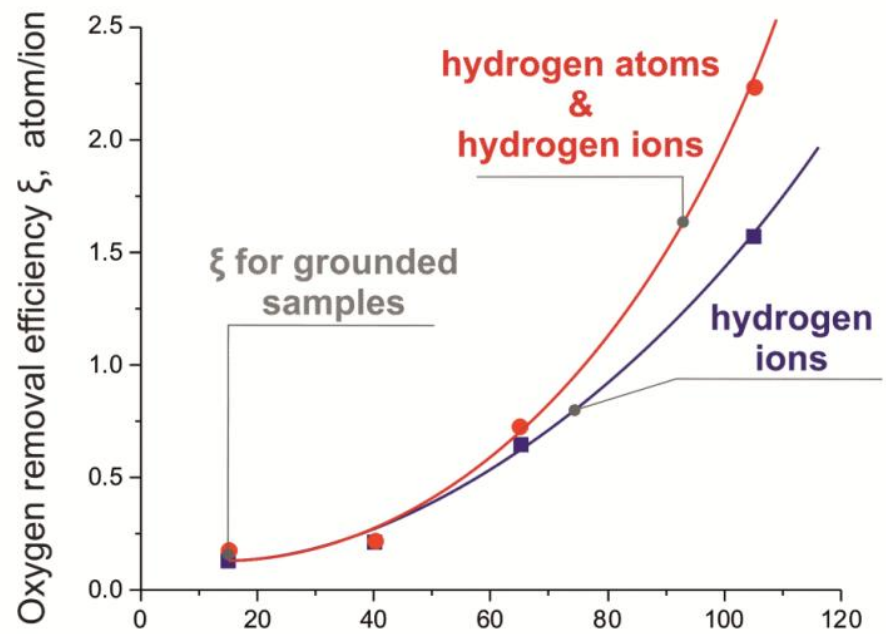

Hydrogen ion energy, eV

Figure 9. 0 atom removal efficiency $\xi$ per ion, as a function of the average ion energy with (red curve) and without (blue curve) additional hydrogen radical flux.

We propose the following mechanism for the observed energydependence for ion-assisted removal of oxygen. For low-energy ions $\left(\mathrm{E}_{\mathrm{i}}<\sim 40 \mathrm{eV}\right)$ where $\xi<0.5$, ion-surface neutralization, which produces hot $\mathrm{H}$ atoms on the surface, is responsible for $\mathrm{O}$ removal, while for higher energies $\left(E_{i}>50 \mathrm{eV}\right)$, chemical sputtering starts to contribute, and eventually dominates.

Arrhenius plots for these two mechanisms are shown in figure 10 . We assume that the surface energy depends on the energy of the incident ions. Or $k T_{s} \approx y E_{i}$ where $y$ is a coefficient that takes into account energy loss due to collisions, stopping processes etc. Taking this assumption, we fit the dependence between oxygen removal 
efficiency and $1 / E_{i}$ in order to find the activation energies for different ruthenium reduction processes.

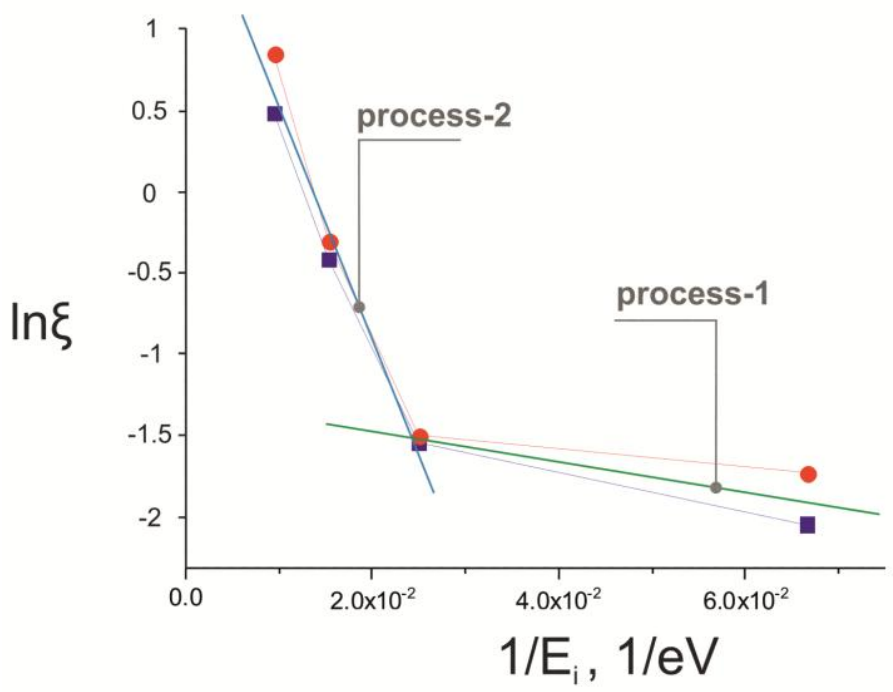

Figure 10. $\ln \xi$ as a function of $1 / E_{i} \sim 1 / T_{s}$ for ions (blue), ions and radicals (red). As one can see there are two different mechanisms for oxygen removal from RuOx: the fist one for low-energy ions labeled as process- 1 and the second for ions of high energy $>40 \mathrm{eV}$ labeled as process-2.

As can be seen in figure 10, two processes play a role in ruthenium reduction under plasma treatment.

process- 1 is described by the equation:

$$
\ln \xi=\ln A_{1}-1.5 \cdot\left(1 / E_{i}\right)
$$

and dominates at low ion energies, while process-2, which occurs at high ion energies, is given by 


$$
\ln \xi=\ln A_{2}-137 \cdot\left(1 / E_{i}\right)
$$

Given that $k T_{s} \approx y E_{i}$, an estimate for $y$ can be found. The Arrhenius equation becomes:

$$
\ln \xi=\ln A_{1}-\left(E_{a} / k T_{S}\right)=\ln A_{1}-\left(E_{a} / \gamma E_{i}\right)
$$

We propose that process- 1 is the removal of oxygen atoms by hydrogen atoms. The excess energy from the ion dissociates the RuO bond, making $\mathrm{OH}$ formation highly probable, a process well known from ref [10]. The activation energy for this process is $0.48 \mathrm{eV}$ [10], which means that $\mathrm{y}=0.32$.

Substituting $y$ into the equation (4) one finds an activation energy of about $44 \mathrm{eV}$. The energy is higher than the binding energy of surface atoms in oxide structure. This implies that the process responsible for ruthenium reduction is not a direct process. Ions with an energy of $44 \mathrm{eV}$ will, as they stop, drive many energetic processes, such as secondary electron emission, which can dissociate more than a single ruthenium oxide bond. At the same time, these processes result in the formation of atomic hydrogen atoms at or just below the surface of the ruthenium oxide. Moreover, volume vacancies could be generated to increase oxygen diffusion to the upper surface layers. Thus, there are many possible indirect processes that may contribute to process-2. Interestingly, the linearity of process- 2 indicates that only a single pathway is dominant.

Thus $\mathrm{H}_{3}{ }^{+}$ions are very effective for removing oxygen from the surface and recovery of $\mathrm{Ru}$ in comparison with $\mathrm{H}$ atoms. However, to evaluate the applicability of in-line plasma etching to EUV lithography, experiments under EUV lithography relevant conditions were performed. 


\subsection{EUV-induced plasma experiments.}

EUV exposures were performed in an UHV chamber, attached to a discharge produced plasma EUV source, which is described in detail in [23]. Briefly, EUV radiation from a tin plasma is refocused by collector optics at the sample position, located in an UHV chamber. The UHV chamber is separated from the main volume by a spectral purity filter (SPF), which is opaque from the deep UV to the visible range and transmits primarily the source's EUV radiation at a wavelength of $13.5 \mathrm{~nm}$. By using differential pumping and the SPF, the vacuum in the UHV chamber is as low as $10^{-6} \mathrm{~Pa}$. The UHV chamber can be filled with different gases to a pressure up to 100 $\mathrm{Pa}$, and the samples can be biased to control the ion energy of the flux incident on the sample surface.

The EUV beam is focused to a diameter of $12 \mathrm{~mm}$ (FWHM $6.3 \mathrm{~mm}$ ) and has a spatially and temporally averaged intensity of $0.13 \mathrm{~W} / \mathrm{cm}^{2}$ in the focus spot. To eliminate most of the scattered EUV radiation, a diaphragm of synthetic mica $(\varnothing=8 \mathrm{~mm}$ ) was placed on top of the samples. The EUV source operates at $1.5 \mathrm{kHz}$, and the energy flux per pulse is $0.085 \mathrm{~mJ} / \mathrm{cm}^{2}$ in a $100 \mathrm{~ns}$ (FWHM) pulse.

Ruthenium films of $25 \mathrm{~nm}$ on a silicon substrate $1 \times 1 \mathrm{~cm}^{2}$ size were oxidized in a plasma reactor to a depth of up to $0.69 \mathrm{~nm}$, according to calibrated XRF measurements. Afterwards they were exposed to EUV in the presence of hydrogen at a pressure of $3 \mathrm{~Pa}$. All exposures were carried out at room temperature with a sample bias voltage of $100 \mathrm{~V}$.

Three sets of experiments was carried out for 2, 5 and 10 million EUV pulses. The total ion charge, measured by the sample current, was used to estimate both the ion-flux incident on the sample surface, and the number of ions formed in the EUV-induced plasma above 
the sample. Thereby, the applied radiation doses of 2-, 5- and $10 \cdot 10^{6}$ pulses corresponded to an integrated ion dose of 6-, 15- and 30.1015 ions at the surface.

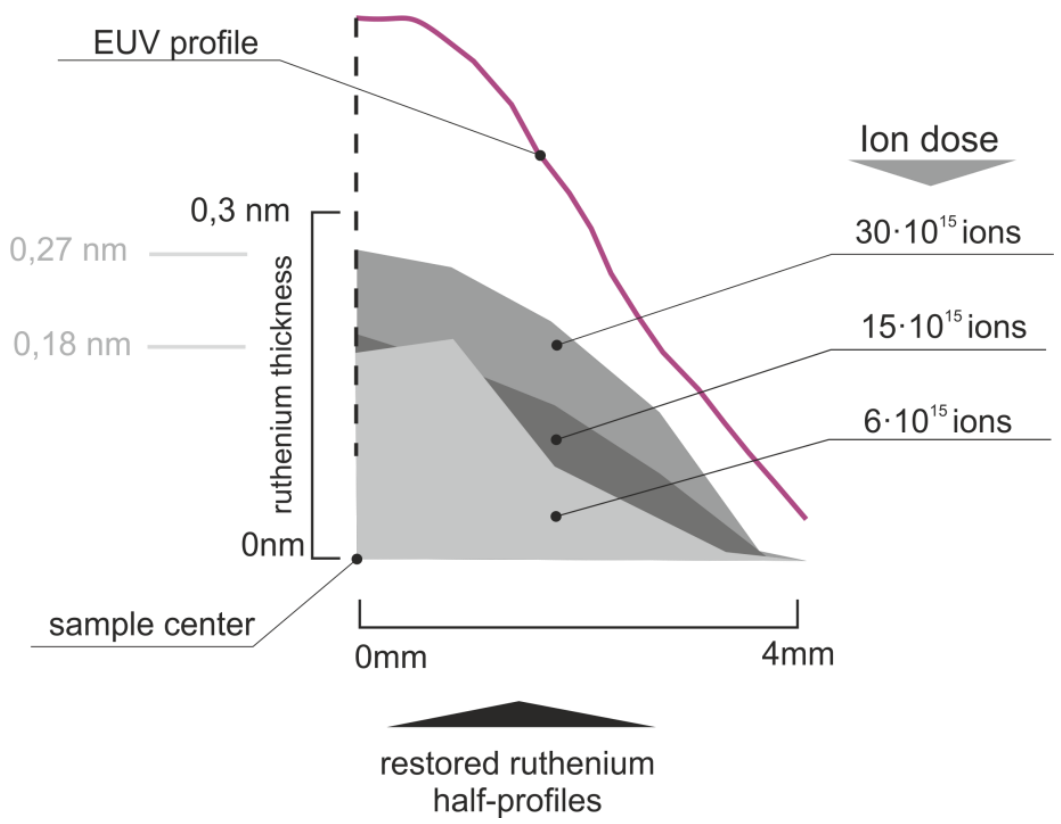

Figure 11. Ruthenium reduction half-profiles for different ion doses (exposure time). Hydrogen ion flux profile depends on the EUV intensity profile. The dependency between the reduction and the spatial profile of the EUV beam is clearly observable.

Ruthenium reduction half-profiles for different ion doses (exposure times) are shown in Figure 11. Hydrogen ion flux profile depends on the EUV intensity profile (it is also shown by line in Figure 11). It is clearly seen that the RuOx reduction profiles correlate well with the profile of the EUV beam that implies the reduction activated by EUVinduced plasma.

Under conditions that are similar to those expected during EUVL, only the surface of the ruthenium is reduced, while the underlying 
oxide is not reduced. Moreover, the dependence on total ion dose is very weak, so the reduction efficiency falls off after the top surface is reduced, as expected. On the other hand, the correlation between the spatial profile of the EUV spot and the spatial profile of the oxide reduction show that there is a clear dependence between $\mathrm{RuO}_{2}$ reduction and ion flux.

Removing underlying layers of oxide requires that hydrogen penetrates, and that the oxygen can defuse to the surface. For ion energies in the range used here, the stopping distance is estimated to be 1-2 nm, which is sufficient to reduce the entire oxide layer. Therefore, we conclude that the reduction of ruthenium is limited by the diffusion of oxygen to the surface.

\subsection{Discussion}

Results of plasma experiments show that one of the reduction processes can be similar to that for hydrogen atoms, which is described in [10] where the oxide removal from ruthenium is described by a stepwise process. In our experiments, it is likely that $\mathrm{RuO}_{2}$ is the predominant oxide [13]. In a $\mathrm{RuO}_{2}$ matrix, hydrogen may be present within the lattice, weakly bound to the $\mathrm{O}_{\text {lattice }}$ and on the surface, adsorbed to the bridge, $\mathrm{O}_{\text {bridge, }}$ between two ruthenium atoms. According to ref [10], the surface of the ruthenium oxide hydrogenates through adsorption. In addition, hydrogen atoms, adsorbed at surface $\mathrm{O}_{\text {bridge }}$ sites, forms hydroxyl groups that are chemically bound to the surface $\left(\mathrm{O}_{\text {bridge }}-\mathrm{H}\right)$. The formation of the $\mathrm{OH}$ bond results in a nearby vacancy:

$$
2\left(\mathrm{O}_{\text {bridge }}-\mathrm{H}\right) \rightarrow\left(\mathrm{O}_{\text {absorb }}-2 \mathrm{H}\right)+\mathrm{O}_{\text {bridge }}+\text { surface vacancy. }
$$

This is followed by water desorption. As a result, oxygen and vacancy concentration gradients form in the oxide film. These gradients are 
perpendicular to the surface and lead to oxygen diffusion from the oxide bulk under the following scheme [10].

$$
\mathrm{O}_{\text {lattice }}+\text { surface vacancy } \rightarrow \mathrm{O}_{\text {bridge }}+\text { vacancy in the lattice. }
$$

As a result, oxygen escapes to the surface, where it can be removed as described earlier.

According to earlier research, vacancies have an important (if not a crucial) role in metal recovery from oxides under the action of hydrogen. In the case of ion bombardment, vacancies are formed directly by ion bombardment, rather than by hydrogen exchange and water desorption (with surface diffusion accounted for). In particular, oxygen bonds are broken on the surface with the resulting formation of the hydroxyl group under the action of a highenergy ion. This, in turn, leads to formation of oxygen vacancies in the surface layer. Nonetheless, at low ion fluxes, the flux may not provide sufficient ions to allow the reaction between hydroxyl groups and hydrogen (either as an ion or a radical) to proceed at high efficiency. Furthermore, at ion energies above $50 \mathrm{eV}$, a significant portion of the flux penetrates the surface and is unable to reduce surface oxide. A combined flux of ions and radicals provides both the energy to activate hydroxyl formation and a supply of reactant. With increased radical concentrations, there is a high probability for the hydroxyl group to react with a radical to form water. Figures 7 and 8 show that this is related to the increased reduction efficiency in the presence of hydrogen atoms on ruthenium oxide.

Once the surface is reduced, further $\mathrm{RuO}_{2}$ reduction is rate limited by two processes: the implantation or diffusion of reactive hydrogen species and the diffusion of oxygen. This naturally leads to a deceleration of the efficiency of oxygen removal from deeper layers. 


\subsection{Conclusions}

Our experiments show that the top atomic layer of ruthenium can be effectively and efficiently reduced from an oxide to a metallic state under hydrogen ion fluxes that are relevant to EUVL. Furthermore, we show that the balance between reduction and oxidation can be effectively controlled by adjusting the partial pressure of hydrogen in the chamber.

Additional experiments demonstrate that the removal of oxide from beneath the surface is more difficult to describe. Rather high energy ions are required to deliver reactive hydrogen species to the buried oxide, while the rate at which oxygen diffuses to the surface is found to be very low. As a result, the reduction rate is most likely limited by the slow diffusion of oxygen through ruthenium. 


\section{References:}

1. International Technology Roadmap for Semiconductors, http://www.itrs.net

2. Banine V, Benschop J 2003 Proc. of SPIE $\mathbf{5 4 0 1} 7861$

3. Al-Ajlony A, Kanjilal A, Catalfano M, Harilal S S, Hassanein A and Rice B 2012 Proc. of SPIE 8322832232.

4. Chen J Q, Louis E, Lee C J, Wormeester H, Kunze R, Schmidt H, Schneider D, Moors R, van Schaik W, Lubomska M, and Bijkerk $F$ 2009 Optics Express 17 16969-16979.

5. Madey T E, Faradzhev N S, Yakshinskiy B V, Edwards N V 2006 Applied Surface Science 253 1691-1708.

6. Hollenshead J and Klebanoff L 2006 J. Vac. Sci. Technol. B 24 6482.

7. Matsunari S, Aoki T, Murakami K, Gomei Y, Terashima S, Takase $\mathrm{H}$, Tanabe $\mathrm{M}$, Watanabe $\mathrm{Y}$, Kakutani $\mathrm{Y}$, Niibe $\mathrm{M}$, and Fukuda $\mathrm{Y}$ 2007 Proc. of SPIE 6517 65172X-8.

8. Niibe M, Koida K, and Kakutani Y 2011 Journal of Vacuum Science \& Technology B 29011030.

9. Oizumi H, Izumi A, Motai K, Nishiyama I, and Namiki A, 2007 Jap. J App. Phys Part 2-Letters \& Express Letters 46 L633-L635.

10. Ugur D, Storm A J, Verberk R, Brouwer J C, Sloof W G 2013 Microelectron. Eng. 110 60-65.

11. Brecelj F, Mozetic M 1990 Vacuum 40(1-2) 177-181.

12. Sawada $Y$, Tamaru $H$, Kogoma $M$, Kawase $M$ and Hashimoto $K$ 1996 J. Phys. D: Appl. Phys. 292539.

13. Iwasaki Y, Izumi A, Tsurumaki H, Namiki A, Oizumi H, Nishiyama I 2007 Applied Surface Science 253 8699-8704.

14. van der Horst R M, Beckers J, Osorio E A, van de Ven T H M and Banine V Y 2015 Plasma Sources Sci Technol. 24035003.

15. Astakhov D I, Goedheer W J, Lee C J, Ivanov V V, Krivtsun V M, Koshelev K N, Lopaev D V, van der Horst R M, Beckers J, Osorio E A and Bijkerk F 2016 J. Phys. D: Appl. Phys. 4929.

16. Dolgov A, Lopaev D, Rachimova T, Kovalev A, Vasil'eva A, Lee C J, Krivtsun V M et al. 2014 J. Phys. D: Appl. Phys. 47065205. 
17. Liu F, Sturm M, Lee C J, Bijkerk F 2016 Surface Science 646 101107.

18. Hollenshead J and Klebanoff L 2006 J. Vac. Sci. Technol. B 24118.

19. Handbook of Optical Constants of Solids Edited by: Edward D. Palik ISBN: 978-0-12-544415-6 (Elsevier Inc).

20. Chung Y M, Lee E M, Masuoka T, and Samson J A R 1993 J. Chem. Phys. 99885.

21. A Dolgov, D Lopaev, CJ Lee, E Zoethout, V Medvedev, O Yakushev 2015 Applied Surface Science 353 708-713.

22. Zyryanov S M, Kovalev A S, Lopaev D V et al. 2011 Plasma Phys. Rep. 37881.

23. A Dolgov, O Yakushev, A Abrikosov, E Snegirev, VM Krivtsun, CJ Lee 2015 Plasma Sources Sci Technol. 24 (3) 035003. 


\section{Summary}

The goal of this work was to characterize the plasma chemistry induced by EUV radiation in a physisorbed layer of molecular species. Model systems were developed, both experimentally and theoretically, allowing contamination scaling laws to be studied. Applied to the case of photolithography, this work focuses on revealing the relevant physics and photochemical processes for cleaning. To highlight important physical processes, contamination and cleaning mechanisms were studied as a function of the surface and plasma environment. The plasma impact on chemistry and on the cap-layer surfaces are discussed in this thesis.

Using an experimental setup that directly reproduces Extreme UVlithography relevant conditions, a numerical model for obtaining the spatio-temporal behavior of EUV-induced plasma evolution was validated. To achieve this, a setup, equipped with Langmuir probes was used to obtain time resolved experimental data. The experimentally obtained current-voltage curves were used to validate the model. The applicability and accuracy of in situ plasma diagnostics is still a subject of discussion. When using Langmuir probes, it must be taken into account that all the quantities extracted from the current-voltage curves are spatially averaged values. The probes average over the sheath that the probe itself creates during the plasma decay. Thus, the probe current-voltage curves may be dominated by changes to the plasma that the presence of the probe induces. As a result, the plasma may be characterized inaccurately.

The actual plasma parameters corresponding to the experimental results are replicated using Particle In Cell-Monte Carlo numerical modeling. For the case of cylindrical symmetry, the model is an accurate instrument for studying plasma formation during the EUV 
pulse and to discover the most important processes during plasma formation and decay. The model was then used in conjunction with experiments to characterize the plasma in the volume above a multilayer mirror.

The knowledge of EUV-induced plasma formation and evolution allows plasma-induced effects and photo-induced effects to be distinguished. The best illustration of the influence of high-energy photons on cleaning mechanisms has been obtained experimentally for amorphous carbon (a-C). By comparing a-C removal in a surface wave discharge (SWD) plasma and an EUV-induced plasma, the physics of cleaning for photoactive hydrogen and noble helium environments were determined. For helium gas, the results for both plasmas were similar, while for hydrogen, an EUV plasma was found to create a larger ratio of atoms to ions at the sample surface. In the EUV-induced hydrogen plasma, the C-atom yield per ion is higher than in the SWD plasma. The presence of high-energy photons leads to more efficient plasma cleaning due to surface activation processes. We refer to this combination of processes as reactive ion sputtering (RIS).

However, in the presence of high-energy photons and ions, contamination is a function of the surface environment. It has been shown that, in the case of a grazing incidence collector for extreme ultraviolet (EUV) lithography, the molecular contamination layer may have physical properties similar those of a diamond-like carbon (DLC) film. Such a DLC contamination layer may require ten times more $\mathrm{H}_{3}{ }^{+}$ions to remove one $\mathrm{C}$ atom during hydrogen plasma treatment. This highlights the importance of understanding the growth of contamination in order to optimize cleaning processes.

Low-temperature hydrogen plasmas can also be effective for reducing oxidized ruthenium top surfaces of a MLM. And that, for 
water-induced oxidation, reduction and oxidation can be balanced by controlling the ratio of the water and hydrogen partial pressures. Our experiment shows that this balance follows a linear relationship, indicating that the chemistry is relatively simple. Follow up experiments on more deeply oxidized $\mathrm{Ru}$ samples showed that reduction of sub-surface oxide is very slow, and may not be reversible using EUV-induced plasma reduction.

Our proof-of-concept experiments demonstrate that an in situ, EUV generated plasma cleaning technology is feasible. The EUV pulse generates a low-temperature plasma, along with photo-induced surface activation. Together these combine to yield a highly reactive environment that quickly and efficiently removes amorphous carbon and reduces ruthenium oxide. We show that by controlling the background gas partial pressure, and by biasing the mirror, the plasma environment and cleaning rates can be controlled with a relatively high level of precision.

Our studies have largely fulfilled the industrially relevant goal of assessing EUV-induced cleaning methods, as listed in Chapter 1:

- EUV-induced plasma cleaning can be performed without interrupting wafer exposure.

- EUV-induced plasma cleaning can be performed at high speed: $3 \mathrm{~nm} / \mathrm{h}$ for carbon and $0.1 \mathrm{~nm} / \mathrm{h}$ for oxygen at ion energies of $\sim 100 \mathrm{eV}$.

- EUV-induced plasma cleaning is highly selective.

$$
\xi_{R u}^{C}=\frac{R_{C}}{R_{R u}}>4 \cdot 10^{3}
$$

where $R_{C}=0.45$ is the carbon removal rate, and $R_{R u}$ is the $\mathrm{Ru}$ removal rate, which is less than $10^{-4}$ at energies over $100 \mathrm{eV}$. 
In conclusion, the studies in this thesis provide better understanding of the mechanism of cleaning (reduction) methods that use EUV radiation generated plasma. The measurement results showed that carbon can be etched, and ruthenium reduced, with high efficiency and high selectivity using hydrogen ions. In performing these experiments, we also showed that numerical modeling, combined with Langmuir probe measurements, can provide accurate spatiotemporal dependent estimates of the plasma characteristics. These can then be used to further refine cleaning methods and to guide the choice of plasma-facing mirror surfaces. 


\section{Valorisation}

The results presented in this thesis provide basic know-how required to adopt radiation-induced plasma surface etching for industrial multilayer mirror cleaning. It has been shown that plasma, generated by pulsed extreme ultraviolet radiation, is a very effective tool for surface carbon cleaning and oxide reduction. Using EUV plasma does not require the installation of additional devices for plasma (or radical) generation, and EUV-induced cleaning could be realized without interrupting production operations, i.e. in situ and in line. This will help to extend the lifetime of EUV optics.

The modified PROTO experimental setup allowed the use of classical plasma probe diagnostics and PIC MC modeling as mutually interdependent techniques to characterize a plasma. This combination of diagnostics and modeling was used to predict the plasma properties of EUV generated plasma under EUVL relevant conditions. Experimental datasets have been used to validate the model. The, thus validated model, in turn, can be used as an independent instrument, capable of obtaining the spatio-temporal evolution of EUV-induced plasma. This approach, depending on the available computational resources, can be more widely applied. However, we note that, currently, the code requires cylindrical symmetry and is restricted to rather dilute plasmas.

This work has practically given a proof of concept for industrial cleaning of multilayer EUV optics. These results in this thesis are intended to make a significant contribution into the further development of EUV optics. 


\section{Appendix 1}

\section{ICP reactor description for chapter 5}

Plasma is generated by ICP (inductively coupled plasma) discharge with a frequency $13.56 \mathrm{MHz}$, excited by 3-turn coil around the quarts tube (see set up) quartz tube (56 $\mathrm{mm}$ inner diameter) at pressure of 100 mTorr.

Samples are placed in ICP downstream and located at RF (12 MHz) biased (capacitively coupled) electrode. Energy of ions incident on the sample surface was controlled by $\mathrm{RF}(12 \mathrm{MHz})$ voltage and negative DC self-bias because of the discharge asymmetry.

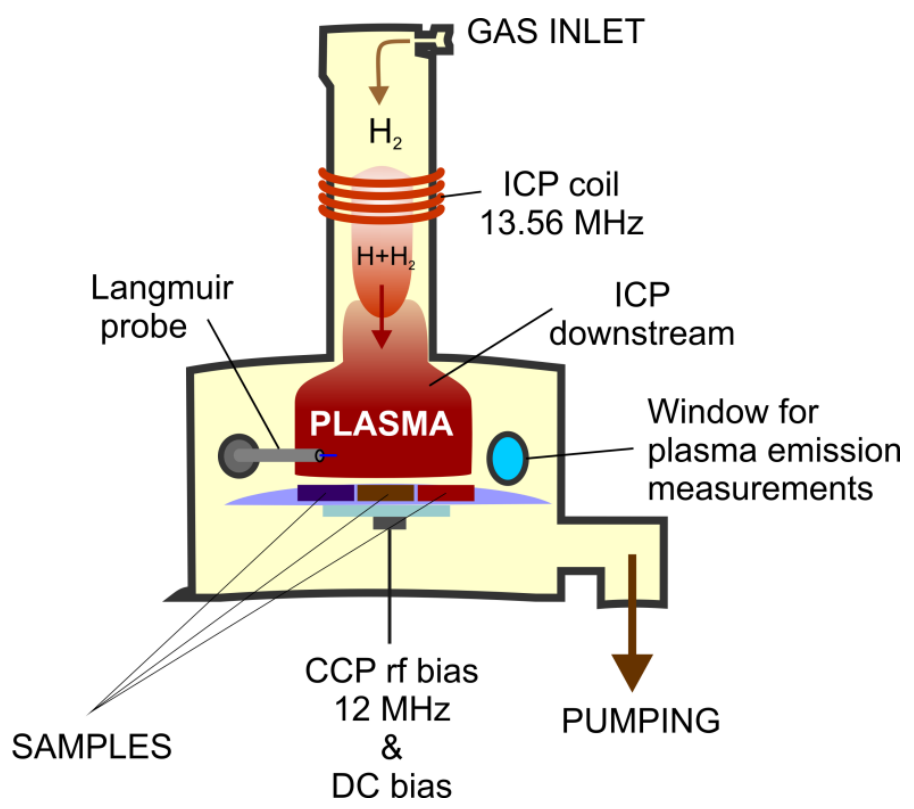

Figure 1. ICP downstream reactor. 
To characterize plasma and thereby conditions on a sample surface we used RF compensated Langmuir probe and emission spectroscopy (actinometry). Actinometry involves using of the optical emission intensity ratios to provide an estimation of atom concentrations in a discharge afterglow and has showed a good reliability [1,2]. The intensity emitted from the plasma species is divided by the emission intensity from actinometer - the gas with a known spectrum and excitation and de-excitation rate constants. As rule this technique is used in low-pressure conditions when collisional deactivation of the emitting atoms is negligible. In this case, we can reasonably assume that the emitting excited state atoms are produced by the electron impact upon the ground-state atoms, taking into account a short lifetime of the excited state. In our case, argon was used as an actinometer. To calculate the excitation rate constants the measured EDDF and the so-called optical excitation cross-sections including the cascading transitions from the upper states to the chosen levels of both $\mathrm{H}$ and Ar atoms were used. Since the measured EEDF was close to maxwellian one, only plasma density, plasma potential, electron temperature as well as ion flux were determined from the probe characteristics measured along the chamber diameter by using the moveable Langmuir probe. These measurements showed uniform plasma in the chamber center where the samples were placed.

$10 \% \operatorname{Ar}$ was added to hydrogen, and transitions $\mathrm{H}\left(3^{2} \mathrm{P}-2^{2} \mathrm{~S}\right)$ and $\operatorname{Ar}\left(2 \mathrm{p}_{1}-1 \mathrm{~s}_{2}\right)$ at the wavelength 656.3 and $750.4 \mathrm{~nm}$, respectively, were observed. The CCP $12 \mathrm{MHz}$ RF biasing (see figure 1) was used to generate optical emission in the afterglow of the main ICP discharge, allowing for actinometric measurements of the $\mathrm{H}$ atom concentration. The difference in measured $\mathrm{H}$ atom concentration with the main $13.56 \mathrm{MHz}$ discharge turned on and off was taken as the $\mathrm{H}$ atom concentration induced by the main discharge. The 
developed discharge system allows producing hydrogen atoms concentrations $\sim 10^{14}-10^{15} \mathrm{~cm}^{-3}$. The flux of hydrogen atoms to the sample surface was calculated as $[H] V_{T} / 4$, where $[H]$ is the concentration of hydrogen atoms above the sample surface, and $V_{T}$ is the thermal velocity of $\mathrm{H}$ atoms. For 100 mTorr of $\mathrm{H}_{2}, 400 \mathrm{~W}$ power of ICP discharge measured ratio atomic to molecule concentrations is $[\mathrm{H}] /\left[\mathrm{H}_{2}\right]=0.115$ and $\mathrm{H}$ atom flux is $f_{\mathrm{H}}=2 \cdot 2 \cdot 10^{19} \mathrm{at} /\left(\mathrm{cm}^{2} \cdot \mathrm{s}\right)$.

The sample surface in plasma is exposed both to hydrogen atoms and ions. Except collector grown carbon two kind of samples were used: Si/Mo multilayer sample with $\sim 70 \mathrm{~nm}$ of amorphous magnetron deposited carbon and silicon wafer with $\sim 20 \mathrm{~nm}$ of amorphous magnetron deposited carbon on top.

\section{References}

1. Gicquel A, Chenevier M, Hassouni K, Tserepi A, and Dubus $M$ 1998 J. Appl. Phys. 837504.

2. Braginsky $O V$, Kovalev A S, Lopaev $D$, Malykhin $E M$, Mankelevich Y A, Rakhimova T V, Rakhimov A T, Vasilieva A N, Zyryanov S M, and Baklanov M R 2010 J. Appl. Phys. 108073303. 


\section{Acknowledgements}

To my life-coach and mentor Konstantin Koshelev: because I owe it all to you. Many Thanks!

With my sincere gratitude to my supervisor Prof. dr. Fred Bijkerk for the support of my Ph.D study, for his patience, motivation and knowledge. I appreciate all his contributions of time, ideas, and funding to make my Ph.D. experience productive and stimulating.

Many thanks to my thesis committee: Prof. dr. ir.H. J. W. Zandvliet, Prof. dr. P. W. H. Pinkse, Prof. dr. ir. V. Y. Banine, Dr. D. V. Lopaev, for their encouragement, insightful comments, and hard questions.

My sincere thanks also goes to my co-supervisor Dr. C. J. Lee for providing me opportunities to do experiments in ASML Lab and leading me to work on exciting tasks. His brilliant remarks and comments were important and vital to thesiswriting process. He knows exactly how to make dry scientific article interesting and juicy. I'm lucky to have him as my mentor.

I thank my fellow labmates in ASML: Feng Liu, Luc Stevens, Edgar Osorio, Malgorzata Pachecka, Rogier van den Bos, Baibhav Mund for the stimulating talks and for all the fun we have had. Also I thank my friends in Moscow State University: Sergey Zyryanov, Alexey Zotovich. They know how to create nice plasma. And I am grateful to Erwin Zoethout and Andrei Nikipelov for the fruitful discussions on my research project. 
I would also like to express my gratitude to all XUV-group colleagues, for their support and kindness. And, especially, many thanks to both group secretaries, Jacqueline and Carin, for their assistance during my PhD period.

In addition, I would like to thank my mates, Maxim Hodak and Dmitry Kuznetsov who took my offer to be paranymphs.

I was happy to get support from my beloved Natasha at the thesis preparation. Thanks a lot.

Special mention goes to Nickolay Stepanenko, for going far beyond the regular friendship. His spiritual strength and wisdom inspired me to be focused on what I'm doing.

I am grateful to my parents and sisters, who are providing me moral and emotional support through my life. And especially my encouraging always interested mom: she was always eager to know what I was doing and how I was proceeding.

I wish I could give a warmest hug to my auntie Natalia. Love you and miss you. Without her this thesis wouldn't be possible. You were taken way too soon.

I am also grateful to all my family members and friends who have supported me along this way. Here is no place to mention all of you, guys. 


\section{Curriculum Vitae}

Alexandr Dolgov was born on 26 July 1985 in Kaunas, USSR. In 1987 family went to Simferopol, Crimea. In this town Alexandr studied at the highschool in a class with emphasis on mathematics and physics. In 2002 he started his education at the Moscow Institute of Physics and Technology (MIPT) in the faculty of Problems of Physics and Energetics. His bachelor's degree work was fulfilled in the Institute of Spectroscopy of the Russian Academy of Sciences under guidance of Professor B. Dumesh. In frames of this work Alexandr designed and constructed a system of automatic frequency generator in the scheme of the NMR spectrometer. In 2008 he successfully got Master's degree with diploma "NMR spectrometer with a second sound method of detecting." In 2010 Alexandr joined the Nanolayer Surfaces and Interfaces (nSI) Group of the FOM Institute DIFFER, Nieuwegein, under the supervision of Prof. dr. Fred Bijkerk. In 2013, the group was relocated to the University of Twente, being part of the later founded Industrial Focus Group XUV Optics, within the MESA+ Institute for Nanotechnology. 